\title{
ANÁLISE INTRABLOCOS DE EXPERIMENTOS EM BLOCOS INCOMPLETOS PARCIALMENTE BALANCEADOS COM ALGUNS TRATAMENTOS COMUNS ADICIONADOS EM CADA BLOCO
}

ANTÔNIO CARLOS DE OLIVEIRA

Orientador: Prof. Dr. DECIO BARBIN

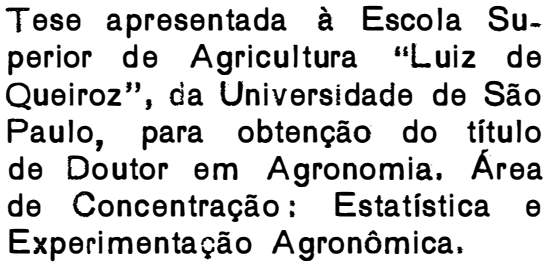

$P \mid R A C I C A B A$

Estado de São Paulo - Brasil

Outubro, 1985 
ii.

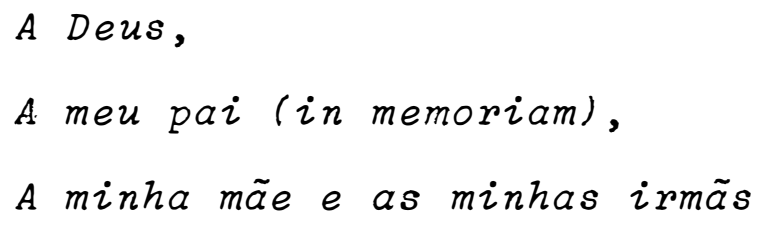

A minha esposa Flávia, A minha filha Paula, DEDICO. 


\section{AGRADECIMENTOS}

Ao Dr. Décio Barbin, Professor Titular do De partamento de Matemática e Estatística da ESALQ/USP, pela valiosa orientação na realização deste trabalho.

Aos Professores do Curso de Pós-Graduação em Estatística e Experimentação Agronômica da ESALQ/USP, na pes soa de seu Coordenador Prof. Dr. Humberto de Campos, pela acolhida e ensinamentos prestados.

Ao Centro Nacional de Pesquisa de Milho e Sor go, da EMBRAPA, pela oportunidade concedida para a realização do Curso.

Ao Dr. Roland Vencovsky, pela revisão do SUMMARY .

A Srta. Maria Izalina Ferreira Alves, pelo trabalho datilográfico e pela amizade.

Aos funcionários do Departamento de Matemāti ca e Estatística da ESALQ/USP, pela amizade e cooperação.

Aos colegas do Curso de Pós-Graduação, pelo convivio amigo. 
1. INTRODUÇÃO $\ldots \ldots \ldots \ldots \ldots \ldots \ldots \ldots \ldots \ldots \ldots \ldots \ldots \ldots \ldots \ldots \ldots \ldots$

2. REVISÃO BIBLIOGRAFICA $\ldots \ldots \ldots \ldots \ldots \ldots \ldots \ldots \ldots \ldots$

2.1 - Ensaios em Blocos Incompletos Balanceados .. 5

2.1 .1 - Definição $\ldots \ldots \ldots \ldots \ldots \ldots \ldots \ldots$. 5

2.1 .2 - Classificação $\ldots \ldots \ldots \ldots \ldots \ldots \ldots 6$

2.1 .3 - Análise de variância intrablocos .. 7

2.2 - Ensaios em Blocos Incompletos Parcialmente Balanceados ...................... 10

2.2.1 - Generalidades .............. 10

2.2 .2 - Classificação $\ldots \ldots \ldots \ldots \ldots \ldots \ldots 16$

2.2.2.1 - Grupo divisivel ....... 16

2.2.2.2 - Triangular $\ldots \ldots \ldots \ldots \ldots 19$

2.2.2.3 - Tipo quadrado latino .... 20

2.2.2.4-Cíclico ............ 21

2.2 .2 .5 - Simples $\ldots \ldots \ldots \ldots \ldots \ldots 23$

2.2 .3 - Construção de delineamentos PBIB .. 29

2.2.4 - Análise de variância intrablocos .. 30

2.2.5 - Variância da estimativa de um contras te entre duas médias de tratamentos 33

2.3 - Ensaios em Blocos Incompletos com Alguns Tra tamentos Adicionais em Cada Bloco 
Pág.

3. DESENVOLVIMENTO TEठ́RICO ..................... 41

3.1 - Introdução ........................ 41

3.2 - Modelo Matemático .................. 43

3.3 - Sistema de Equações Normais ........... 45

3.4 - Estimação do Efeito da Média Geral ....... 49

3.5 - Estimação dos Efeitos de Tratamentos Ajustá dos para Blocos ......................... 50

3.5 .1 - Método clássico ............. 50

3.5 .2 - Método simplificado .......... 53

3.5.2.1 - Estimação dos efeitos dos tratamentos adicionais ... 53

3.5.2.2 - Estimação dos efeitos dos tratamentos originais com m classes de associados .. 55

3.5.2.3 - Estimação dos efeitos dos tratamentos originais com duas classes de associados

3.5.2.4 - Caso especial: Estimação dos efeitos dos tratamenmentos originais em delineamento $\mathrm{BIB} \quad \ldots . . . \ldots$....

3.6 - Propriedades das Soluções do Sistema de Equa ções Normais com Efeitos de Blocos Eliminados 74 3.6 .1 - Esperança matemática de $\tilde{\tau} \ldots \ldots \ldots 75$ 3.6 .2 - Matriz de dispersão para $\underset{\tau}{ } \ldots \ldots \ldots 77$ 
Pág.

3.7 - Somas de Quadrados $\ldots \ldots \ldots \ldots \ldots \ldots \ldots \ldots \ldots$

3.8 - Esperanças Matemáticas das Somas de Quadrados 88 3.8.1 - Esperança matemática da sQm̂ $\ldots \ldots .88$ 3.8 .2 - Esperança matemática de $\tilde{\tau}^{\prime} Q \quad \ldots \ldots .99$

3.8 .3 - Esperança matemática de $B^{\prime} K^{-1} B-C_{0} 91$

3.8 .4 - Esperança matemática de $\underset{\sim}{y}{ }_{\sim}^{y}-C_{0} \ldots 93$

3.8 .5 - Esperança matemática da SQResíduo .. 94

3.8.6 - Esperança matemática da sQTad. ... 95

3.8.7 - Esperança matemática da SQTipos ... 97

3.8 .8 - Esperança matemática da SQTor.(aj.) 100

3.9 - Distribuição das Formas Quadráticas ...... 102

3.9.1 - Distribuição da $\operatorname{sQTaj} \cdot / \sigma^{2} \ldots \ldots \ldots 103$

3.9.2 - Distribuição da SQTad./ $\sigma^{2} \ldots \ldots \ldots 105$

3.9 .3 - Distribuição da SQTipos $/ \sigma^{2} \ldots \ldots \ldots 107$

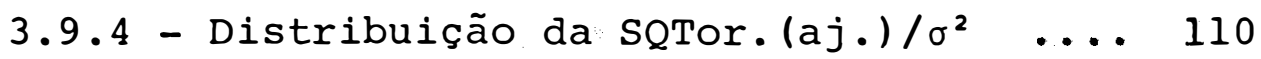

3.9 .5 - Distribuição da SQBlocos/ $/ \sigma^{2} \ldots \ldots \ldots 113$

3.9 .6 - Distribuição da SQResiduo/ $\sigma^{2} \ldots \ldots .115$

3.9.7 - Distribuição do quociente (SOTaj./n $\left./ \mathrm{n}_{1}\right) /$ $\left(\mathrm{S} Q R / \mathrm{n}_{6}\right) \quad \ldots \ldots \ldots \ldots \ldots \ldots \ldots \ldots \ldots \ldots \ldots \ldots \ldots$

3.9 .8 - Distribuição do quociente $\left(\right.$ SQTad. $\left./ \mathrm{n}_{2}\right) /$ $\left(\mathrm{SQR} / \mathrm{n}_{6}\right) \quad \ldots \ldots \ldots \ldots \ldots \ldots \ldots \ldots \ldots \ldots$

3.9.9 - Distribuição do quociente (SQTipos $\left./ \mathrm{n}_{3}\right) /$ $\left(S Q R / n_{6}\right) \ldots \ldots \ldots \ldots \ldots \ldots \ldots \ldots \ldots \ldots \ldots \ldots \ldots$ 
Pág.

3.9.10 - Distribuição do quociente (SQTor.aj./n $\mathrm{n}_{4}$ )/ $\left(\mathrm{SQR} / \mathrm{n}_{6}\right) \quad \ldots \ldots \ldots \ldots \ldots \ldots \ldots \ldots \ldots \ldots \ldots \ldots \ldots \ldots \ldots \ldots$

3.10 - Estimadores das Médias de Tratamentos, Ajustadas para Blocos .................. 124

3.11 - Quadro da Análise de Variância ......... 125

3.12 - Variância da Estimativa de um Contraste entre Duas Médias de Tratamentos ......... 127

3.13 - Testes de Significâncias ............... 130 3.13.1 - Teste de significância para tratamentos ajustados para blocos ..... 130

3.13.2 - Teste de significância para tratamentos adicionais ............ 131

3.13.3 - Teste de significância para tiños de tratamentos ............... 132

3.13.4 - Teste de significância para tratamentos originais ajustados para blo $\cos \ldots \ldots \ldots \ldots \ldots \ldots \ldots \ldots \ldots \ldots \ldots \ldots \ldots \ldots$

4. ILUSTRAÇÃO DO MÉTODO PROPOSTO .............. 135

5. CONCLUSÕES $\ldots \ldots \ldots \ldots \ldots \ldots \ldots \ldots \ldots \ldots \ldots \ldots \ldots \ldots \ldots \ldots$

6. REFERENCIAS BIBLIOGRÁFICAS ................. 150 
ANÁLISE INTRABLOCOS DE EXPERIMENTOS EM BLOCOS INCOMPLETOS PARCIALMENTE BALANCEADOS COM ALGUNS TRATAMENTOS COMUNS ADICIONADOS EM CADA BLOCO

Autor:

Orientador:
Antonio Carlos de Oliveira Prof. Dr. Décio Barbin

No presente trabalho considera-se o caso de um ensaio em blocos incompletos parcialmente balanceados, aumentado pela adição de alguns tratamentos comuns a todos os blocos. Os tratamentos do delineamento inicial são desig nados de "tratamentos originais", e, aqueles que foram adicionados, de "tratamentos adicionais". O objetivo foi apresentar um método geral de análise para esse tipo de ensaio. Os parâmetros do delineamento inicial foram definidos como:

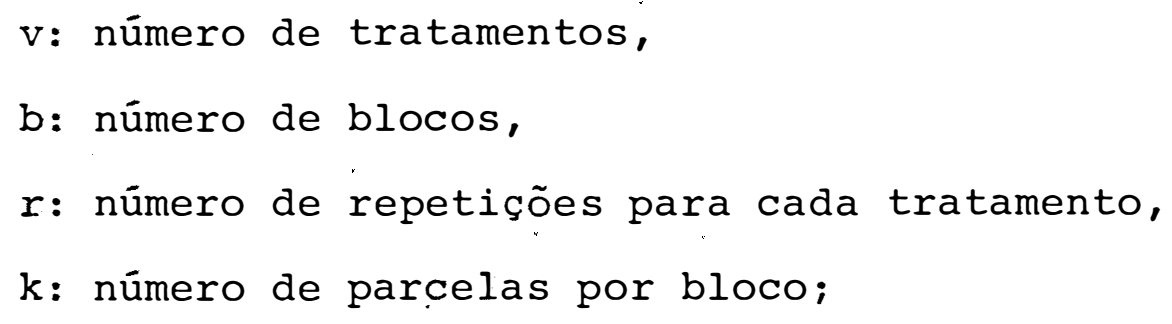


e ainda $\lambda_{1}, \lambda_{2}, \ldots, \lambda_{m}, n_{1}, n_{2}, \ldots, n_{m}, p_{j k}^{i}(i, j, k=1,2, \ldots$ ...,m), definidos conforme BOSE e NAIR (1939).

A inclusão de $c$ tratamentos adicionais em ca da bloco do experimento, resultou em um delineamento aumentado, com os seguintes parâmetros:

$$
\begin{aligned}
& \mathrm{v}^{\prime}=\mathrm{v}+\mathrm{C} \text { (número total de tratamentos), } \\
& \text { b: número de blocos, } \\
& k^{\prime}=k+c \text { (número de parcelas por bloco), } \\
& k^{\prime} b=n \text { (número total de parcelas), }
\end{aligned}
$$

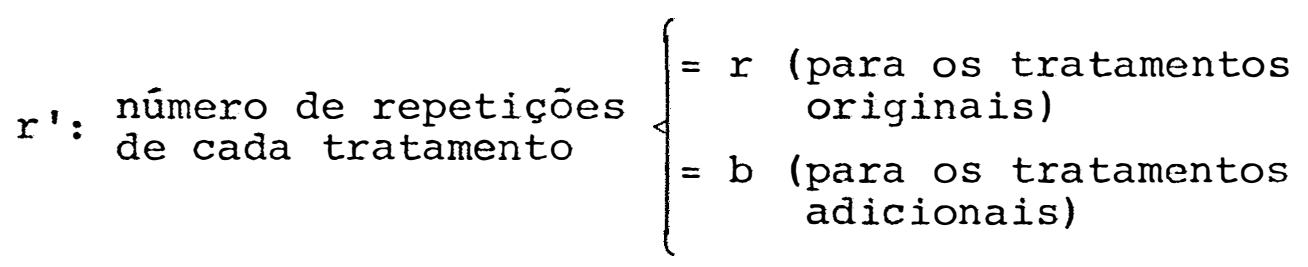

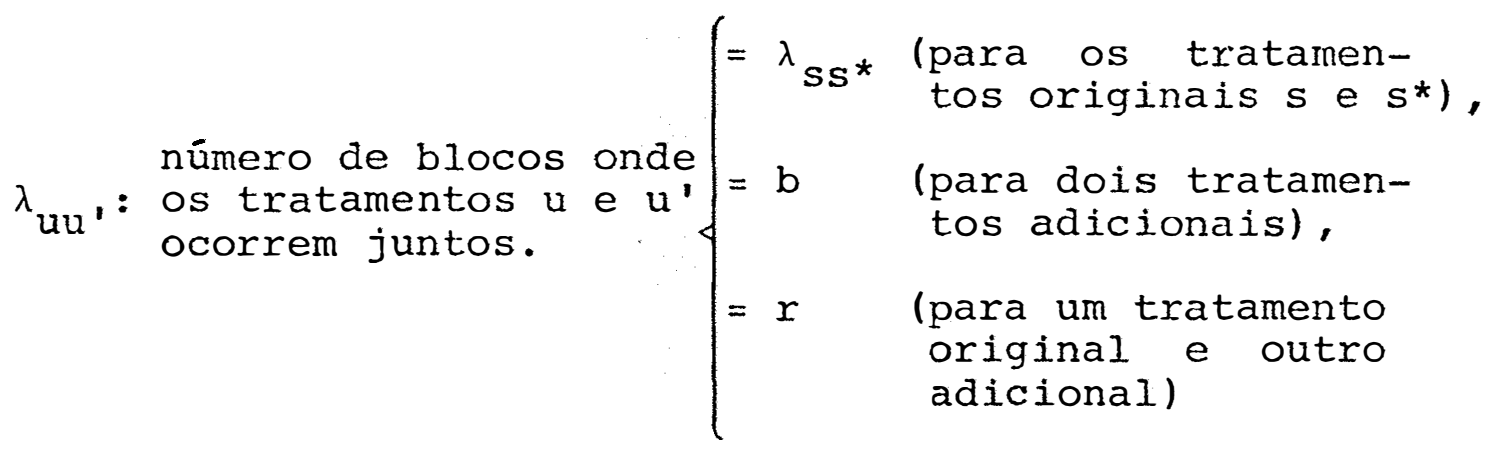

$$
\begin{aligned}
& \text { o modelo matemático adotado foi o seguinte: } \\
& y_{u h}=m+t_{u}+b_{h}+e_{u h} \text {, }
\end{aligned}
$$

onde, 
$y_{u h}$ é a observação do u-ésimo tratamento no h-ésimo bloco;

m è a média geral;

$t_{u}$ é o efeito do u-ésimo tratamento $\left(u=1,2, \ldots, v^{\prime}\right)$;

$b_{h}$ é o efeito do $h$-ésimo bloco $(h=1,2, \ldots, b)$;

$e_{u h}$ é o erro experimental associado à observação $Y_{u h}$ ' onde se supõe que os $e_{u h}$ 's são inảependen tes e normalmente distribuídos, com média zero e variância $\sigma^{2}$.

Sob as condições anteriores são determinados: O sistema de equações normais, os estimadores dos efei tos dos parâmetros, a matriz de dispersão para os efeitos dos tratamentos, as somas de quadrados e suas esperancas ma temáticas; e ainda, as distribuições das formas quadráticas e a variância da estimativa de um contraste entre duas médias de tratamentos.

São apresentados também os estimadores dos efeitos dos tratamentos originais, a soma de quadrados desses tratamentos e a variância da estimativa de um contraste entre duas médias, para o caso em que o delineamento inicial é em blocos incompletos balanceados.

Um exemplo numérico é apresentado para ilustrar o método proposto. 
INTRABLOCK ANALYSIS OF EXPERIMENTS IN PARTIALLY

BALANCED INCOMPLETE BJOCKS WITH SOME COMMONS TREATMENTS ADDED TO EACH BLOCK

Author: Antonio Carlos de Oliveira

Adviser: Prof. Dr. Décio Barbin

A statistical investigation was conducted for the case in which experiments are designed in partially balanced incomplete blocks, but some common treatments are added to each block. Treatments of the basic or initial design were designated as "original treatments" and the other ones as "additional treatments". The main objective was to develop a general method of analysis for this specific kind of experiments.

The following parameters were considered as pertinent to the initial design:

$\mathrm{v}$ : number of treatments,

$\mathrm{b}$ : number of blocks,

$r$ : number of replication of each treatment,

k: number of plots per block; 
and $\lambda_{1}, \lambda_{2}, \ldots, \lambda_{n}, n_{1}, n_{2}, \ldots, n_{m}, p_{j k}^{i}(i, j, k=1,2, \ldots, m)$, defined according to BOSE and NAIR (1939).

The inclusion of $c$ additional treatments to each block, resulted in augmented design, with the following parameters:

$$
\begin{aligned}
& v^{\prime}=v+c \text { (total number of treatments), } \\
& b \text { : number of blocks, } \\
& k^{\prime}=k+c \text { (number of plots per block), } \\
& k^{\prime} b=n \text { (total number of plots), } \\
& \text { r': } \begin{array}{l}
\text { number of replications } \\
\text { of each treatment }
\end{array}\left\{\begin{array}{cc}
=x & \text { (for original treatments } \\
=b & \text { (for additional treat- } \\
\text { ments) }
\end{array}\right. \\
& \text { number of blocks in } \\
& \lambda_{u u^{\prime}}: \text { which treatments } u \\
& \text { and } u^{\prime} \text { occur together } \\
& \begin{cases}=\lambda_{S^{*}} & \text { (for two original } \\
& \text { treatments) } \\
=\mathrm{b} \quad & \text { (for two additional } \\
& \text { treatments) } \\
& \text { (for an original and } \\
& \text { an additional treat } \\
& \text { ment) }\end{cases}
\end{aligned}
$$

The following mathematical model was considered:

$$
y_{u h}=m+t_{u}+b_{h}+e_{u h}
$$


where,

$y_{\text {uh }}$ denotes the observation of the $u^{\text {th }}$ treatment in the $\mathrm{h}^{\text {th }}$ block;

$m$ denotes the effect of the general mean;

$t_{u}$ denotes the $u^{\text {th }}$ treatment effect $\left(u=1,2, \ldots, v^{\prime}\right)$;

$b_{h}$ denotes the $h^{\text {th }}$ block effect $(h=1,2, \ldots, b)$;

$e_{u h}$ is a random error component with expectation ze ro and variance $\sigma^{2}$, the $e_{u h}$ 's being distributed inảependently.

Under the given conditions, the solution of the normal equations, the dispersion matrix for treatment effects, the sum of squares, the expectations of sum of squares, the distributions of the quadratic forms, and the variances of the treatment differences, were obtained. The estimators of the original treatment effects, its sums of squares, and the variances of the treatments differences were also obtained, when the balanced incomplete block design is used as the initial design.

A numerical example is used to illustrate the proposed method. 


\section{INTRODUÇÃO}

Quando um grande nümero de tratamentos deve ser comparado em ensaios de campo, o que acontece frequentemente na pesquisa agrícola em programas de avaliação e melhoramento de plantas, o pesquisador defronta-se com o problema da distribuição dos tratamentos na área experimen tal. Este aspecto reveste-se de maior importância nesse tí po de experimento, pela dificuldade em conseguir-se áreas relativamente homogêneas, principalmente quanto à fertilidade do solo, que proporcionem comparações mais precisas.

A eficiência dos delineamentos de FISHER (1925), amplamente conhecidos como blocos casualizados e quađrado latino, quando adotados nessa situação, é sensivelmente prejudicada, como ressaltam BOSE e NAIR (1939). Pa 
ra estes autores a eficiência diminui à medida que o nümero de parcelas por bloco cresce a partir de dez ou doze. Além disso, um quadrado latino contendo o total de tratamentos é em geral impossível, não só pelo elevado número de repetições requerido, mas também pela grande quantidade de sementes que seria necessária, que quase sempre é limitada. O uso de blocos casualizados, ainda que possível, não é satisfatório quando cada bloco contém todos os tratamentos, visto que é improvável a eliminação das diferenças de fertilidade eficientemente, conforme relata YATES (1936).

Com o objetivo de eliminar-se a heterogeneidade dentro dos blocos, e assim contornar os problemas men cionados, YATES (1936) introduziu o delineamento de blocos incompletos, que é obtido mođificando-se os blocos completos casualizados com respeito ao tipo de arranjo dos trata mentos, ou seja, a restrição de que o nümero de unidades experimentais por bloco seja igual ao número de tratamentos ê dispensada. Neste tipo de arranjo modificado, o nüme ro de unidades por bloco é fixo, sendo menor do que o número de tratamentos, e estes são distribuídos nos blocos de tal forma que quaisquer dois tratamentos ocorram num bloco com igual frequência. Nessa situação todas as comparações entre tratamentos têm a mesma precisão, e o delineamento é chamado de blocos incompletos balanceados, e abreviadamen- 
te de BIB ("Balanced incomplete block design") por diversos autores.

Os delineamentos do tipo BIB, segundo BOSE e NAIR (1939), são os mais eficientes entre todos os delinea mentos incompletos, mas são possiveis em apenas um número limitado de casos. Além disso, há situações em que a utilí zação de tais delineamentos é praticamente impossivel, devido ao excessivo número de repetições que é requerido. Por exemplo, um reticulado quadrado com 81 tratamentos exigiria 10 repetições para que a condição de balanceamento fos se satisfeita, o que em geral, não é viável na prática.

Para contornar esse problema, BOSE e NAIR (1939) introduziram os delineamentos em blocos incompletos parcialmente balanceados, denominados de PBIB ("Partially balanced incomplete block design"), nos quais não há restrição quanto ao número de repetições. Estes delineamentos vêm sendo utilizados frequentemente na pesquisa agrícola quando o número de tratamentos a ser comparado é grande.

Há ainda situações em que se recomenda o uso de determinados tratamentos em todos os blocos. Isto ocorre, por exemplo, conforme salienta FERREIRA (1980), quando se deseja comparar alguns tratamentos novos com outros, de comportamento já conhecido, e que atuam como uma espécie de controle ou testemunha. 
No presente trabalho considera-se o caso em que os tratamentos novos, denominados aqui de tratamentos originais, são distribuídos na área experimental conforme um delineamento PBIB, e as testemunhas, denominadas de tra tamentos adicionais, são incluídas em cada bloco do ensaio. o objetivo é apresentar um método geral de análise intrablocos para esse tipo de ensaio. Um exemplo numérico também é apresentado para ilustrar o método proposto. 


\section{REVISÃO BIBLIOGRAFICA}

2.1 - Ensaios em Blocos Incompletos Balanceados

$$
2.1 .1 \text { - Definição }
$$

Segundo YATES (1936), um arranjo de v tratamentos em b blocos de k unidades experimentais, é chamado delineamento de blocos incompletos balanceados (BIB), se ele satisfaz às seguintes condições:

i) cada bloco contém k diferentes tratamentos;

ii) cada tratamento ocorre em $r$ blocos;

iii) quaisquer dois tratamentos ocorrem'juntos em $\lambda$ blo cos.

Pela definição de delineamentos BIB, pode-se verificar, como mostra YATES (1936), que:

$$
x v=b k \quad \text { e } \quad \lambda=r(k-1) /(v-1) \text {. }
$$


Estas igualdades definem as condições de balanceamento de um delineamento BIB.

\section{1 .2 - Classificação}

Em geral, os delineamentos BIB são classificados em cinco diferentes tipos:

i) TIPO I: Os blocos podem ser agrupados em repetições, ou seja, em $r$ conjuntos de $n$ blocos cada, tal que cada conjunto forma uma repetição completa de todos os tratamentos.

ii) TIPO II: Os blocos não podem ser reunidos em repetições, mas podem ser reunidos em grupos de repeti ções.

iii) TIPO III: Os blocos não podem ser reunidos em repetições nem em grupos de repetições.

iv) TIPO IV: O número de tratamentos é igual ao nümero de blocos.

v) TIPO V: Pequenos experimentos.

Os delineamentos TIPO I e TIPO III são denominados por NAIR (1944) de "resolvable.designs" e "non-resolvable designs", respectivamente. Os demais tipos são men 
cionados por COCHRAN e COX (1957), sendo que o TIPO IV tam bém é conhecido como delineamentó BIB simétrico.

Uma classe importante de delineamentos TIPOI é constituída dos chamados reticulados quadrados ("Squares lattices" ou "Two-dimensional lattices"), assim denominados por PIMENTEL GOMES (1976). Nesses delineamentos o núme ro de tratamentos deve ser um quadrado perfeito e o tamanho do bloco é a raiz quadrada desse número, ou seja, v = $k^{2}$.

Nos reticulados quadrados balanceados todo par de tratamentos ocorre no mesmo bloco uma única vez. Co mo consequência, a condição de balanceamento $\lambda(v-1)=r(k-1)$ conduz a k+l repetições. Esses delineamentos, no entanto, só são viáveis se $k$ é um número primo ou uma potência de primo, conforme mencionam COCHRAN e COX (1957).

\subsection{3 - Análise de variância intrablocos}

Como ressalta IEMMA (1981), os autores são unânimes no que se refere à decomposição da soma de quadra dos total na análise de variância. A análise é feita como se o delineamento fosse em blocos casualizados completos, com a diferença de que a soma de quadrados de tratamentos é ajustada para efeitos de blocos. Nos delineamentos TIPOI 
e TIPO II a soma de quadrados de blocos pode ainda ser decomposta.

Para a estimação dos efeitos de tratamentos ajustados para blocos, os autores em geral consideram o se guinte sistema de equações normais:

$$
C \underline{\tilde{\tau}}=\underset{\sim}{ },
$$

onde $\mathrm{C}$ é uma matriz singular, de dimensões $\mathrm{v} \times \mathrm{v}$ e caracte ristica $(v-1)$, com os elementos:

$$
c_{i i}= \begin{cases}r\left(1-\frac{1}{k}\right), & \text { se } i=i^{\prime} \\ -\lambda / k & , \text { se } i \neq i^{\prime}\end{cases}
$$

$\underline{\tau}$ é o vetor dos efeitos dos tratamentos estimados, de dimensões v $\times 1$, e

$\underline{Q}$ é um vetor de dimensões $\mathrm{v} x \mathrm{l}$ com os elementos $Q_{i}$ $(i=1,2, \ldots, v)$ definidos por:

$$
Q_{i}=T_{i}-\frac{1}{k} A_{i},
$$

sendo $\mathrm{T}_{i}$ o total das unidades experimentais que contêm 0 i-ésimo tratamento, e $A_{i}$ o total dos blocos que contêm esse mesmo tratamento.

A solução do sistema de equações ë apresentạ da por PIMENTEL GOMES (1967) considerando uma matriz de 
de restrições $\mathrm{A}$ de dimensões $\mathrm{v} \times \mathrm{v}$, tal que $\underset{\sim}{\mathrm{i}}=\underset{\sim}{\mathrm{O}}$ Fazendo então $C-A=M$, matriz esta não singular, $O$ autor apresenta a solução para $\bar{\tau}$, ou seja:

$$
\tilde{\tau}=M^{-1} \underline{\sim} \ldots
$$

Uma vez estimado o vetor dos efeitos de tratamentos, a soma de quadrados para tratamentos ajustada pa ra blocos é dada por:

$$
\operatorname{SQT}(a j \cdot)=\tilde{I}^{\prime} \stackrel{O}{=} .
$$

Como relata CHAKRABARTI (1962), as equações normais $C_{\underset{\sim}{\tilde{I}}}=\underset{\sim}{Q}$ são satisfeitas para $\underset{\sim}{\tilde{\tau}}=(k / v \lambda) \underset{\sim}{Q}$. Logo, $a$ soma de quadrados para tratamentos, ajustada para blocos, pode ser colocada na seguinte forma:

$$
\operatorname{SQT}(\operatorname{aj} \cdot)=\frac{k}{v^{\lambda}} \stackrel{Q}{\prime}^{\prime} \underset{\sim}{Q}
$$

CHAKRABARTI (1962) menciona ainda que o melhor estimador de $\tau_{i}-\tau_{i}$, é $(k / v \lambda)\left(Q_{i}-Q_{i}\right)$, com variân cia $\sigma^{2}(2 k / v \lambda)$, ou seja:

$$
\begin{aligned}
V\left(\tilde{\tau}_{i}-\tilde{\tau}_{i}\right) & =V\left(\frac{k}{V \lambda}\left(Q_{i}-Q_{i}\right)\right) \\
& =\frac{2 \sigma^{2}}{r} \frac{k r}{v \lambda} \\
& =\frac{2 \sigma^{2}}{r} \frac{k r(k-1)}{V \lambda(k-1)}
\end{aligned}
$$




$$
V\left(\hat{\tau}_{i}-\hat{\tau}_{i},\right)=\frac{2 \sigma^{2}}{r} \frac{1-\frac{1}{v}}{1-\frac{1}{k}} .
$$

A variância correspondente no caso de blocos casualizados é $2 \sigma^{2} / \mathrm{r}$ (supondo o mesmo $\sigma^{2}$ para ambos os casos). Assim, a eficiência dos delineamentos BIB em relação a blocos casualizados é definida por diversos autores como:

$$
E=\frac{v \lambda}{k r}=\frac{1-\frac{1}{k}}{1-\frac{1}{v}}<1 .
$$

2.2 - Ensaios em Blocos Incompletos Parcialmente Balanceados

\subsection{1 - Generalidades}

Para BOSE e NAIR (1939) um delineamento em blocos incompletos é parcialmente balanceado, se ele satis faz às seguintes condições:

i) Os tratamentos são agrupados em b blocos de $k$ unidades cada, sendo as unidades do mesmo bloco constituidas de diferentes tratamentos.

ii) Há $v$ tratamentos que ocorrem em $r$ blocos.

iii) Fixando-se qualquer tratamento; os demais podem ser divididos em $m$ grupos de tamanhos $n_{1}, n_{2}, \ldots, n_{m}$ 
tal que os tratamentos do i-ésimo grupo ocorram juntos com o dado tratamento em $\lambda_{i}$ blocos. Os tratamentos do i-ésimo grupo são chamados de i-ésimos associados do tratamento em questão e os valores de $\mathrm{n}_{1}, \mathrm{n}_{2}, \ldots, \mathrm{n}_{\mathrm{m}} ; \lambda_{1}, \lambda_{2}, \ldots, \lambda_{\mathrm{m}}$ são independentes do tratamento inicialmente considerado.

iv) Se o tratamento $\mathrm{A}$ é i-ésimo associado de $\mathrm{B}$, então o tratamento B é i-ésimo associado de A. Se A e B são i-ésimos associados, então o número de tratamen tos comuns aos j-ésimos associados de A e aos k-ési mos associados de $\mathrm{B}$ é $\mathrm{p}_{j \mathrm{j}}^{\mathrm{i}}$, e é independente do par de tratamentos considerado. Também, $p_{j k}^{i}=p_{k j}^{i}$.

Os parâmetros $v, b, r, k, \lambda_{1}, \lambda_{2}, \ldots, \lambda_{m}, n_{1}$, $\mathrm{n}_{2}, \ldots, \mathrm{n}_{\mathrm{m}}$ são chamados "parâmetros do primeiro tipo", e os $\underline{p}_{j k}^{i}(i, j, k=1,2, \ldots, m)$ de "parâmetros do segunđo tipo". Assim, há $2 \mathrm{~m}+4$ parâmetros do primeiro tipo e $\mathrm{m}^{2}(\mathrm{~m}+1) / 2 \mathrm{pa}-$ râmetros do segundo tipo (visto que $p_{j k}^{i}=p_{k j}^{i}$ ).

BOSE e NAIR (1939) mencionam ainda que quando $m=1$, o delineamento se transforma em blocos incompletos balanceados. O mesmo acontece quando $\lambda_{1}=\lambda_{2}=\ldots=\lambda_{m}$ ' conforme relata NAIR (1944).

Na definição original de delineamento PBIBos parâmetros $\lambda_{1}, \lambda_{2}, \ldots, \lambda_{m}$ eram considerados como sendo di 
ferentes. A modificação introduzida por Nair e Rao (1942), citados por BOSE e SHIMAMOTO (1952), permite que alguns $\lambda$ 's sejam iguais.

BOSE e CLATWORTHY (1955) mostram que é desnecessāria a suposição de que todos os parâmetros $p_{j k}^{i}$ sejam constantes no caso de delineamentos com duas classes de associados. Segundo os autores, se se assume que $n_{1}, n_{2}$, $\mathrm{p}_{11}^{1}$ e $\mathrm{p}_{11}^{2}$ são constantes, todas as outras condições automa ticamente são satisfeitas.

Segundo observam BOSE e NAIR (1939), se veri ficam as seguintes relações entre os parâmetros:

i) $\mathrm{vr}=\mathrm{bk}$;

ii) $n_{1}+n_{2}+\ldots+n_{m}=v-1$;

iii) $n_{1} \lambda_{1}+n_{2} \lambda_{2}+\ldots+n_{m} \lambda_{m}=r(k-1)$;

$$
\text { iv) } \begin{aligned}
\sum_{k=1}^{m} p_{j k}^{i} & =n_{j} \quad(\text { se } i \neq j) ; \\
& =n_{j}-1 \quad(\text { se } i=j) ;
\end{aligned}
$$

iv) $n_{i} p_{j k}^{i}=n_{j} p_{i k}^{j}$.

BOSE e NAIR (1939) mostram que se os parâmetros do primeiro tipo satisfazem aos itens (i), (ii) e (iii), então em vista das relações (iv) e (v), somente $m\left(m^{2}-l\right) / 6$ dos parâmetros do segundo tipo são independentes. Esses au 
tores relatam que os parâmetros $p_{j k}^{i}$ podem ser arranjados em matrizes simétricas $P_{i}=\left(p_{j k}^{i}\right)$, para $i=1,2, \ldots, m$, onde $p_{j k}^{i}$ é o $(j, k)$-ésimo termo da matriz $\mathrm{P}_{i}$.

Os delineamentos parcialmente balanceados com apenas duas classes de associados $(m=2)$ são de especial in teresse, conforme salientam BOSE e SHIMAмOTO (1952). Nesse caso há 8 parâmetros do primeiro tipo $\left(v, b, r, k, \lambda_{1}, \lambda_{2}\right.$, $\mathrm{n}_{1}$ e $\mathrm{n}_{2}$ ) conectados pelas relações (i), (ii) e (iii), e 6 parâmetros do segundo tipo $\left(p_{j k}^{i} ; i, j, k=1,2\right)$ conectados pelas relações (iv) e (v). Os parâmetros do segundo tipo podem ser apresentados então como elementos de duas matrizes simétricas:

$$
p_{1}=\left(\begin{array}{ll}
p_{11}^{1} & p_{12}^{1} \\
p_{21}^{1} & p_{22}^{1}
\end{array}\right), p_{2}=\left(\begin{array}{ll}
p_{11}^{2} & p_{12}^{2} \\
p_{21}^{2} & p_{22}^{2}
\end{array}\right)
$$

BOSE e SHIMAMOTO (1952) relatam que a relação de associação entre os tratamentos nos delineamentos PBIB pode ser estabelecida através de uma regra, que infor ma que tipo de associados dois dados tratamentos são. Em particular, quando $m=2$, a regra especifica se dois tratamentos são primeiros ou segundos associados. A regra deve ser tal que as condições impostas na definição dos delinea mentos PBIB sejam satisfeitas. Como ilustração os autores consideram um experimento em que $v=10$ e $m=2$. Os 10 trata- 
mentos $a, b, c, d, e, f, g, h, i, j$ são então arranjados no seguinte esquema de associação:

$\begin{array}{lllll}* & \mathrm{a} & \mathrm{b} & \mathrm{c} & \mathrm{d} \\ \mathrm{a} & * & \mathrm{e} & \mathrm{f} & \mathrm{g} \\ \mathrm{b} & \mathrm{e} & * & \mathrm{~h} & \\ \mathrm{c} & \mathrm{f} & \mathrm{h} & * & \\ \mathrm{~d} & \mathrm{~g} & \mathrm{i} & j & \text { * }\end{array}$

A regra de associação é a seguinte: Dois tra tamentos são primeiros associados se e somente se eles ocor rem juntos na mesma coluna do esquema, e são segundos asso ciados em caso contrário. Pode-se verificar então que cada tratamento tem 6 primeiros associados $\left(n_{1}=6\right)$ e 3 segundos associados $\left(\mathrm{n}_{2}=3\right)$. Exemplificando, os primeiros e segundos associados de $\underline{a}, \underline{b}$ e $\underline{i}$ são dados a seguir:

Tratamento
a

$\mathrm{b}$

i
Primeiros associados

$$
b, c, d, e, f, g
$$

$a, c, d, e, h, i$

$b, e, h, d, g, j$
Segundos associados

$$
\begin{aligned}
& i, j, h \\
& f, g, j \\
& a, c, f
\end{aligned}
$$

Os tratamentos a e $\underline{b}$ são primeiros associados. O nümero de tratamentos que são primeiros associados de a e também primeiros associados de $\underline{b}$ é 3 ; tais tratamen tos são $\underline{c}, \underline{d}$ e e. Portanto, $p_{1}^{1}=3$. Pode-se verificar que 
com quaisquer dois tratamentos que sejam primeiros associa dos obtêm-se exatamente 3 tratamentos que são primeiros a sociados de ambos. Isto verifica a condição de constância de $\mathrm{p}_{11}^{\mathrm{I}}$ para qualquer par de tratamentos. Por outro lado, $\underline{\mathrm{f}}$ e $q$ são os únicos tratamentos comuns aos primeiros associa dos de a e segundos associados de $\underline{b}$. Logo, $\mathrm{p}_{12}^{1}=2$, que é constante para qualquer par de tratamentos considerado. 0 mesmo se verifica para os outros $p_{j k}^{i}$ 's.

Os parâmetros $p_{j k}^{i}$ 's, no exemplo, quando apre sentados na forma proposta por BOSE e NAIR (1939), são

$$
P_{1}=\left(\begin{array}{ll}
3 & 2 \\
2 & 1
\end{array}\right), \quad P_{2}=\left(\begin{array}{ll}
4 & 2 \\
2 & 0
\end{array}\right)
$$

BOSE e SHIMAMOTO (1952) relatam ainda que pa ra obter-se um delineamento baseado no esquema de associação anteriormente mencionado, deve-se arranjar os 10 trata mentos dentro dos blocos, satisfazendo as condições da definição de delineamentos PBIB. O delineamento resultante é classificado como triangular. 


\subsection{2 - Classificação}

Para BOSE e SHIMAMOTO (1952) a divisão dos delineamentos PBIB em um pequeno número de tipos distintos simplifica a computação numérica, bem como a interpretação dos resultados. Assim, dependendo da natureza do esquema de associação entre os tratamentos, classificam os delineamen tos com duas classes de associados, em cinco diferentes tí pos: Grupo divisível, triangular, quadrado latino, cúbico e simples. A definição de cada tipo e os respectivos esque mas de associação, segundo esses autores, são apresentados a seguir.

\subsubsection{1 - Grupo divisivel}

Um delineamento PBIB com duas classes de associados é do tipo grupo divisivel (GD) se há v=mn tratamentos, e estes podem ser divididos em $m$ grupos de $n$ tratamentos cada, de forma que quaisquer dois tratamentos do mes mo grupo são primeiros associados e dois tratamentos em gru pos diferentes são segundos associados. O esquema de associação pode ser apresentado pela reunião dos tratamentos em um retângulo, onde as colunas formam os grupos. Nesse caso tem-se que: 


$$
\begin{aligned}
& n_{1}=n-1, n_{2}=n(m-1), \\
& (n-1) \lambda_{1}+n(m-1) \lambda_{2}=r(k-1),
\end{aligned}
$$

e os parâmetros do segundo tipo são determinados unicamente por $m$ e $n$, como a seguir:

$$
P_{1}=\left(\begin{array}{cc}
n-2 & 0 \\
0 & n(m-1)
\end{array}\right), \quad P_{2}=\left(\begin{array}{cc}
0 & n-1 \\
n-1 & n(m-2)
\end{array}\right)
$$

Dependendo dos valores dos $\lambda$ 's, os delineamentos GD são subdivididos por BOSE e CONNOR (1952) em três classes:
i) Singular (S), se $r=\lambda_{1}$;
ii) Semiregular (SR), se $r>\lambda_{1}$ e $r k=v \lambda_{2}$;
iii) Regular (R), se $r>\lambda_{1}$ e $r k>v \lambda_{2}$.

BOSE E SHIMAMOTO (1952) relatam que um delineamento GD singular é sempre derivado de um delineamento BIB, substituindo-se cada tratamento por um grupo de $\mathrm{n}$ tra tamentos. Os grupos resultantes formam o esquema de associação entre os tratamentos. Como ilustração os autores con sideram 0 delineamento BIB com os parâmetros $\mathrm{v}^{*}=\mathrm{b}^{*}=7, \mathrm{r}^{*}=$ $k^{*}=3, \lambda^{*}=1$. O plano experimental para esse caso é dado a seguir, onde as colunas representam os blocos: 


$\begin{array}{lllllll}\text { a } & \text { b } & c & \text { d } & \text { e } & f & g \\ \text { b } & \text { c } & \text { d } & \text { e } & f & g & a \\ \text { d } & \text { e } & \text { f } & g & a & b & c\end{array}$

Tomando-se $n=2$, pode-se então substituir o tratamento a por $\underline{a}_{-1}$ e $\underline{a}_{2}$, fazendo-se o mesmo para os outros tratamentos. Obtém-se assim o delineamento GD singular com os parâmetros

$$
v=14, \quad b=7, \quad r=3, \quad k=6, \quad \lambda_{1}=3, \quad \lambda_{2}=1, \quad m=7, \quad n=2 \text {, }
$$

e o seguinte plano experimental

BLOCO I $\quad$ BIJOCO $2 \quad$ BLOCO $3 \quad$ BI,OCO $4 \quad$ BLOCO $5 \quad$ BIJOCO $6 \quad$ BLOCO 7

$\begin{array}{ccccccc}\mathrm{a}_{1} & \mathrm{~b}_{1} & \mathrm{c}_{1} & \mathrm{~d}_{1} & \mathrm{e}_{1} & \mathrm{f}_{1} & \mathrm{~g}_{1} \\ \mathrm{a}_{2} & \mathrm{~b}_{2} & \mathrm{c}_{2} & \mathrm{~d}_{2} & \mathrm{e}_{2} & \mathrm{f}_{2} & \mathrm{~g}_{2} \\ \mathrm{~b}_{1} & \mathrm{c}_{1} & \mathrm{~d}_{1} & \mathrm{e}_{1} & \mathrm{f}_{1} & \mathrm{~g}_{1} & \mathrm{a}_{1} \\ \mathrm{~b}_{2} & \mathrm{c}_{2} & \mathrm{~d}_{2} & \mathrm{e}_{2} & \mathrm{f}_{2} & \mathrm{~g}_{2} & \mathrm{a}_{2} \\ \mathrm{~d}_{1} & \mathrm{e}_{1} & \mathrm{f}_{1} & \mathrm{~g}_{1} & \mathrm{a}_{1} & \mathrm{~b}_{1} & \mathrm{c}_{1} \\ \mathrm{~d}_{2} & \mathrm{e}_{2} & \mathrm{f}_{2} & \mathrm{~g}_{2} & \mathrm{a}_{2} & \mathrm{~b}_{2} & \mathrm{c}_{2}\end{array}$

o esquema de associação é

$$
\begin{array}{lllllll}
a_{1} & b_{1} & c_{1} & d_{1} & e_{1} & f_{1} & g_{1} \\
a_{2} & b_{2} & c_{2} & d_{2} & e_{2} & f_{2} & g_{2}
\end{array}
$$

Os autores mencionam ainda que em geral, em correspondência ao delineamento BIB com parāmetros v*, b夫, 
$\mathrm{r}^{*}, \mathrm{k}^{*}, \lambda^{*}$, tem-se um delineamento GD singular com os parâa metros

$$
v=n v^{*}, \quad b=b^{*}, \quad r=r^{*}, \quad k=n k^{*}, \quad \lambda_{1}=r, \quad \lambda_{2}=\lambda^{*}, \quad m=v^{*}, n \text {. }
$$

\subsubsection{2 - Triangular}

Neste caso tem-se $v=n(n-1) / 2$, e o esquema de associação dos tratamentos é uma matriz de $\mathrm{n}$ linhas e $\mathrm{n}$ co lunas com as seguintes propriedades:

i) As posições na diagonal principal são vazias.

ii) As $n(n-1) / 2$ posições acima da diagonal principal são ocupadas pelos números $1,2, \ldots, n(n-1) / 2$, cor respondentes aos tratamentos.

iii) As $n(n-1) / 2$ posições abaixo da diagonal principal são ocupadas, tal que a matriz seja simétrica.

iv) Dois tratamentos que ocorram na mesma coluna são primeiros associados, enquanto que em caso contrário são segundos associados.

No caso geral verificam-se as seguintes rela

ções :

$$
\begin{gathered}
n_{1}=2 n-4, \quad n_{2}=(n-2)(n-3) / 2 \\
P_{1}=\left(\begin{array}{cc}
n-2 & n-3 \\
n-3 & (n-3)(n-4) / 2
\end{array}\right), \quad P_{2}=\left(\begin{array}{cc}
4 & 2 n-8 \\
2 n-8 & (n-4)(n-5) / 2
\end{array}\right)
\end{gathered}
$$


BOSE e NAIR (1939) mencionam que um certo nú mero de delineamentos PBIB pode ser obtido, tomando-se em um delineamento BIB, os blocos como tratamentos e estes co mo blocos. SHRIKHANDE (1952) ressalta que esse processo é sempre válido para delineamentos BIB com parâmetros $\mathrm{v}^{*}, \mathrm{~b}^{*}$, $r^{*}, k^{*}, \lambda^{*}$ nos seguintes casos:
(a) $\lambda^{*}=1$;
(b) $r^{*}=k, k^{*}=k-2, \lambda^{*}=2$.

Segundo BOSE e SHIMAMOTO (1952), os delineamentos PBIB, correspondentes ao caso (b), são do tipo trian gular com os seguintes parâmetros:

$$
\begin{gathered}
\mathrm{v}=\mathrm{k}(\mathrm{k}-1) / 2, \quad \mathrm{~b}=(\mathrm{k}-1)(\mathrm{k}-2) / 2, \quad \mathrm{r}=\mathrm{k}-2, \mathrm{k}=\mathrm{k}, \\
\lambda_{1}=1, \quad \lambda_{2}=2 ; \\
\mathrm{n}_{1}=2 \mathrm{k}-4, \quad \mathrm{n}_{2}=(\mathrm{k}-2)(\mathrm{k}-3) / 2 ; \\
\mathrm{P}_{1}=\left(\begin{array}{cc}
\mathrm{k}-2 & \mathrm{k}-3 \\
\mathrm{k}-3 & (\mathrm{k}-3)(\mathrm{k}-4) / 2
\end{array}\right), \quad \mathrm{P}_{2}=\left(\begin{array}{cc}
4 & 2 \mathrm{k}-8 \\
2 \mathrm{k}-8 & (\mathrm{k}-4)(\mathrm{k}-5) / 2
\end{array}\right) \\
2.2 .2 .3-\mathrm{Tipo} \text { quadrado latino. }
\end{gathered}
$$

Um delineamento PBIB com duas classes de associados é do tipo quadrado latino com i restrições $\left(L_{i}\right)$ se os $\mathrm{V}=\mathrm{s}^{2}$ tratamentos satisfazem ao seguinte esquema de as- 
sociação: Os $s^{2}$ tratamentos são arranjados em um conjunto quadrado s x s e i-2 quadrados latinos, mutuamente ortogonais, são superpostos. Dois tratamentos são primeiros associados se e somente se eles ocorrem na mesma linha ou co luna do conjunto, ou nas posições ocupadas pela mesma letra em qualquer dos quadrados latinos. Em outros casos eles são segundos associados. As seguintes relações se verificam:

$$
\begin{gathered}
n_{1}=i(s-1) \quad, \quad n_{2}=(s-1)(s-i+1), \\
P_{1}=\left(\begin{array}{cc}
i^{2}-3 i+s & (i-1)(s-i+1) \\
(i-1)(s-i+1) & (s-i)(s-i+1)
\end{array}\right), P_{2}=\left(\begin{array}{cc}
i(i-1) & i(s-i) \\
i(s-i) & (s-i)^{2}+i-2
\end{array}\right)
\end{gathered}
$$

$$
2.2 .2 .4-\text { Ciclico }
$$

Um delineamento PBIB com duas classes de associados é chamado cíclico (C) se o conjunto de primeiros associados do i-ésimo tratamento é $\left(i+d_{1}, i+d_{2}, \ldots, i+d_{n_{1}}\right)$ módulo $v$, onde os d elementos satisfazem às seguintes condições :

i) Os d elementos são todos diferentes entre si, e $0<d_{j}<v$ para $j=1,2, \ldots, n_{1}$.

ii) Entre as $n_{1}\left(n_{1}-1\right)$ diferenças $d_{j}-d_{j},\left(j, j^{\prime}=1,2, \ldots\right.$, $\left.n_{1}, j \neq j '\right)$ reduzidas a módulo $v$, cada um dos elemen 
tos $d_{1}, d_{2}, \ldots, d_{n_{1}}$ ocorre a vezes, enquanto cada um dos elementos $e_{1}, e_{2}, \ldots, e_{n_{2}}$ ocorre $\beta$ vezes, onde $d_{1}, d_{2}, \ldots, d_{n_{1}}, e_{1}, e_{2}, \ldots, e_{n_{2}}$ são $v-1$ ele mentos diferentes entre si. E necessário que

$$
n_{1} \alpha+n_{2} \beta=n_{1}\left(n_{1}-1\right)
$$

os parâmetros $p_{j k}^{i}$ são dados por

$$
P_{1}=\left(\begin{array}{cc}
\alpha & n_{1}-\alpha-1 \\
n_{1}-\alpha-1 & n_{2}-n_{1}+\alpha+1
\end{array}\right), P_{2}=\left(\begin{array}{cc}
\beta & n_{3}-\beta \\
n_{1}-\beta & n_{2}-n_{1}+\beta-1
\end{array}\right)
$$

Esses parâmetros são apresentados por RAGHAVARAO (1971) na seguinte forma:

$$
\begin{gathered}
v=4 t+1 \quad, n_{1}=n_{2}=2 t, \\
P_{1}=\left(\begin{array}{ll}
t-1 & t \\
t & t
\end{array}\right), P_{2}=\left(\begin{array}{ll}
t & t \\
t & t-1
\end{array}\right)
\end{gathered}
$$

BOSE e SHIMAMOTO (1952) apresentam uma ilustrạão de delineamento do tipo cíclico considerando os parâmetros $v=13, b=13, r=3, k=3, \lambda_{1}=1, \lambda_{2}=0, n_{1}=6, n_{2}=6, \alpha=2$, $B=3$. O esquema de associação, definido pelo conjunto de d's, é 


$$
d_{1}=2, d_{2}=5, d_{3}=6, d_{4}=7, d_{5}=8, d_{6}=11,
$$

e o bloco inicial é $(1,3,9)$. Todos os blocos são obtidos a partir do bloco inicial adicionando-se j-l a todo tratamento $(j=1,2, \ldots, v)$, com redução à módulo $v$.

Os autores observam que entre as diferenças $\mathrm{d}_{j}-\mathrm{d}_{j}{ }^{\prime}$, reduzidas a módulo 13 , cada d ocorre duas vezes, en quanto que qualquer outro inteiro diferente de zero e menor que 13 ocorre três vezes. Os associados para um dado tratamento são facilmente determinados. Assim, os primeiros associados de 4 são os tratamentos $6,9,10,11,12$ e 2. Considerando o bloco inicial $(1,3,9)$, o delineamento completo é dado por (as colunas são os blocos):

$\begin{array}{rrrrrrrrrrrrrr}1 & 2 & 3 & 4 & 5 & 6 & 7 & 8 & 9 & 10 & 11 & 12 & 13 & \text { Rep. 1 } \\ 3 & 4 & 5 & 6 & 7 & 8 & 9 & 10 & 11 & 12 & 13 & 1 & 2 & \text { Rep. } 2 \\ 9 & 10 & 11 & 12 & 13 & 1 & 2 & 3 & 4 & 5 & 6 & 7 & 8 & \text { Rep. 3 }\end{array}$

$$
2.2 .2 .5-\text { Simples }
$$

O delineamento PBIB com duas classes de asso ciados é chamado de simples se
a) $\lambda_{1} \neq 0, \lambda_{2}=0 \quad$ ou
b) $\lambda_{1}=0, \lambda_{2} \neq 0$.

O caso b pode ser trocado para o caso (a) mu dando-se a designação dos primeiros e segundos associados. Em geral o caso (a) é tomado como padrão. Um delineamento 
do tipo simples pode também pertencer a um dos outros tipos já mencionados.

Ainda com respeito à classificação, os delineamentos PBIB são subdivididos por NAIR (1944), seguindo o mesmo princípio empregado nos delineamentos BIB, em dois tipos:

a) "Resolvable design": Os blocos podem ser agrupados em repetições, isto é, em $r$ conjuntos de $n$ blocos cada.

b) "Non-resolvable desian": Os blocos não podem ser agrupados em repetições.

Os delineamentos onde os blocos podem ser agrupados em repetições ("resolvable designs") formam uma importante classe de delineamentos PBIB. Entre eles destacam-se os reticulados quadrados ("square lattices") e, conforme NAIR (1951, 1952), os reticulados retangulares simples ("Simple rectangular lattice") e alguns reticulados retangulares triplos ("Triple rectangular lattice")。 COCHRAN e COX (1957) classificam os reticula dos quadrados parcialmente balanceados em cinco diferentes tipos, e para cada tipo fazem os seguintes comentários: 
i) Reticulado simples (duas repetições)

Neste caso são usadas apenas duas repetições. E possivel assim 0 uso de reticulados parcialmente balanceados para a avaliação de 36,100 e 144 tratamentos, o que não seria possível no caso de delineamentos balanceados.

Para 9 e 16 tratamentos é impossivel que os reticulados simples sejam mais precisos do que blocos casualizados, a menos que a variação entre blocos incompletos seja maior quando comparada com a variação dentro dos mesmos blocos. Além disso os números de graus de liberdade para a estimação do erro experimental nos dois casos são somente 4 e 9, respectivamente, contra 9 e 16 para blocos casualizados.

\section{ii) Reticulado triplo (três repetições)}

Neste caso, das $k+1$ repetiçõe's do delineamen to balanceado, três repetições são usadas. As mesmas precauções mencionadas para reticulado simples, devem ser observadas aqui com a utilização de 9 tratamentos.

\section{iii) Quatro repetições}

Este delineamento pode ser obtido duplicando-se o reticulado simples ou usando-se um reticulado quá- 
druplo, ou seja, as 4 primeiras repetições do reticulado balanceado.

iv) Cinco repetições

De um conjunto balanceado as primeiras 5 repetições são tomadas. Não há delineamentos desse tipo para $9,36,100$ ou 144 tratamentos.

v) Maior nümero de repetições

Delineamentos balanceados podem ser usados para os seguintes números de tratamentos e repetições; 25, $6 ; 49,8 ; 64,9 ; 81,10$. Para 9 tratamentos em 8 ou 12 repetições, 16 tratamentos em 10 repetições, e 25 tratamentos em 12 repetições, o plano para delineamento balanceado seria repetido.

Em outros casos as seguintes recomendações são feitas:

Seis repetições: Usar o reticulado triplo duas vezes. Oito repetições: Usar o reticulado simples quatro vezes ou o reticulado quádruplo duas vezes.

Nove repetições: Usar o reticulado triplo trēs vezes. Dez repetições: Usar o reticulado simples cinco vezes ou o reticulado quíntuplo duas vezes. 
RAGHAVARAO (1971) salienta que os delineamen tos reticulados quadrados, parcialmente balanceados, são do tipo quadrado latino com $i$ restricões $\left(L_{i}\right)$. o autor classi fica esses delineamentos em reticulados simples ("Simple lattice") ou reticulado duplo ("Double lattice"), quando $i=2$; reticulado triplo ("Triple lattice") quando $i=3$; reticulado quádruplo ("Quadruple lattice"), quando $i=4$; e a sim por diante. Observa ainda que se $n$ é o número de vezes que o arranjo básico é repetido, obtém-se então um delinea mento com os parâmetros

$$
v=s^{2}, \quad b=n s i, \quad r=n i, \quad k=s, \quad \lambda_{1}=n, \quad \lambda_{2}=0 .
$$

HARSHBARGER (1949) relata que nos reticulados retangulares simples $\mathrm{v}=\mathrm{p}(\mathrm{p}-1)$, e os $\mathrm{p}(\mathrm{p}-1)$ tratamentos são identificados por $x$ e $y(x \neq y, x, y=1,2, \ldots, p)$. Todos os tratamentos com o mesmo valor para $\mathrm{x}$ formam $0 \mathrm{x}$-ési mo bloco da primeira repetição, e todos os tratamentos com o mesmo valor de y formam o y-ésimo bloco da segunda repetição.

NAIR (1951) mostra que se $p \geqq 4$, o delineamento reticulado retargular simples, forma um delineamento PBIB com quatro classes de associados, com os parâmetros

$$
\begin{aligned}
& \mathrm{v}=\mathrm{p}(\mathrm{p}-1), \mathrm{k}=\mathrm{p}-1, \mathrm{r}=2, \mathrm{~b}=2 \mathrm{p}, \lambda_{1}=1, \lambda_{2}=0=\lambda_{3}=\lambda_{4}, \\
& \mathrm{n}_{1}=2(\mathrm{p}-2), \mathrm{n}_{2}=(\mathrm{p}-2)(\mathrm{p}-3), \mathrm{n}_{3}=2(\mathrm{p}-2), \mathrm{n}_{4}=1 .
\end{aligned}
$$




\begin{tabular}{|c|c|c|c|c|c|c|c|c|c|}
\hline \multirow{4}{*}{$P_{1}=$} & $p-3$ & $p-3$ & 1 & 0 & \multirow{4}{*}{, $P_{2}=$} & 2 & $2(p-4)$ & 2 & 0 \\
\hline & $p-3$ & $(p-3)(p-4)$ & $p-3$ & 0 & & $2(p-4)$ & $(p-4)(p-5)$ & $2(p-4)$ & 1 \\
\hline & 1 & $p-3$ & $p-3$ & 1 & & 2 & $2(p-4)$ & 2 & 0 \\
\hline & 0 & 0 & 1 & 0 & & 0 & 1 & 0 & 0 \\
\hline \multirow{4}{*}{$3=$} & 1 & $p-3$ & $p-3$ & 1) & \multirow{4}{*}{, $P_{4}=$} & 0 & 0 & $2(p-2)$ & 0 \\
\hline & $p-3$ & $(p-3)(p-4)$ & $p-3$ & 0 & & 0 & $(p-2)(p-3)$ & 0 & 0 \\
\hline & $p-3$ & $p-3$ & 1 & 0 & & $2(p-2)$ & 0 & 0 & 0 \\
\hline & 1 & 0 & 0 & 0 & & 0 & 0 & 0 & 0 \\
\hline
\end{tabular}

No caso dos reticulados retangulares triplos, HARSHBARGER (1949) menciona que há $v=p(p-1)$ tratamentos, e cada tratamento pode ser identificado pelas três letras or denadas $(x, y, z)(x \neq y, x \neq z, y \neq z ; x, y, z=1,2, \ldots, p)$. Os tratamentos com o mesmo valor $x$ formam 0 x-ésimo bloco da prí meira repetição, os tratamentos com o mesmo valor para y formam o y-ésimo bloco da segunda repetição, e os tratamen tos com o mesmo valor para $z$ formam o z-ésimo bloco da ter ceira repetição.

NAIR (1951) mostra que os delineamentos reti culados retangulares triplos, são parcialmente balanceađos com os parâmetros

$$
\mathrm{v}=6, \quad \mathrm{~b}=9, \quad \mathrm{r}=3, \quad \mathrm{k}=2, \lambda_{1}=1, \lambda_{2}=0, \mathrm{n}_{1}=3, \mathrm{n}_{2}=2,
$$




$$
P_{1}=\left(\begin{array}{ll}
0 & 2 \\
2 & 0
\end{array}\right), \quad P_{2}=\left(\begin{array}{ll}
3 & 0 \\
0 & 1
\end{array}\right)
$$

quando $\mathrm{p}=3$, e com os parâmetros

$$
\mathrm{v}=12=\mathrm{b}, \quad \mathrm{r}=3=\mathrm{k}, \quad \lambda_{1}=1, \quad \lambda_{2}=0=\lambda_{3}, \mathrm{n}_{1}=6, \mathrm{n}_{2}=3, \mathrm{n}_{3}=2,
$$

quando $\mathrm{p}=4$, mas eles não são delineamentos PBIB para $\mathrm{p} \geq 5$.

\subsection{3 - Construção de delineamentos PBIB}

BOSE e NAIR (1939), baseando-se em diferentes métodos de construção, apresentam uma série de delinea mentos em blocos incompletos parcialmente balanceados com $r \leq 10$ e $k \leq 10$.

Alguns teoremas, relativos à construção de de lineamentos do tipo grupo divisivel, são apresentados por RAGHAVARAO (1971) com base nos estudos de BOSE, SHRIKHANDE e BHATTACHARYA (1953).

RAGHAVARAO (1971) menciona que a teoria da geometria finita é de grande utilidade na construção de de lineamentos PBIB, e cita alguns autores que usaram essa teo ria: Bose e Chakrarti. (1966); Clatworthy (1964); Dai e Feng (1964); Raychaudhuri (1962, 1965); Seiden (1966); Wan (1964, 1965); Wan e Yang (1964); Yamamoto, Fukuda e Hamada (1966) e Yang (1965). Cita ainda vārios autores que utilizaram ou 
tros métodos: Addelman e bush (1964), Archbold e Johnson (1956), Bose e Clatworthy (1955), Clatworthy (1967), dentre outros.

\subsection{4 - Análise de variância intrablocos}

De modo análogo aos delineamentos BIB, os au tores em geral apresentam a análise de variância como se o delineamento fosse em blocos casualizados completos, com a diferença de que a soma de quadrados de tratamentos é ajustada para efeitos de blocos, que é calculada segundo BO SE e NAIR (1939) da seguinte forma:

$$
\operatorname{SQT}(\operatorname{aj} .)=\sum_{i=1}^{v} \hat{t}_{i} Q_{i}
$$

onde $t_{i}$ é o efeito estimado do tratamento $i \quad(i=1,2, \ldots, v)$ e $Q_{i}$ é definido como

$$
Q_{i}=T_{i}-\frac{1}{k} A_{i},
$$

sendo $\mathrm{T}_{i} \circ$ total das unidades experimentais que contém 0 i-ésimo tratamento, e $A_{i} \circ$ total dos blocos que contêm esse tratamento.

Para os delineamentos com duas classes de as sociados $(m=2)$, BOSE e NAIR (1939) apresentam a seguinte expressão para $\hat{t}_{i}$ : 


$$
\hat{t}_{i}=\frac{k}{\Delta}\left[B_{22} Q_{i}-B_{12} S_{1}\left(Q_{i}\right)\right], i=1,2, \ldots, v
$$

onde,

$$
\begin{aligned}
S_{1}\left(Q_{i}\right) & \text { é a soma dos } Q^{\prime} s \text { correspondentes aos } n_{1} \text { tratamen } \\
& \text { tos que são primeiros associados do i-ésimo tra- } \\
& \text { tamento; } \\
A_{12}= & r(k-1)+\lambda_{2} ; \\
A_{22}= & \left(\lambda_{2}-\lambda_{1}\right) P_{12}^{2} ; \\
B_{12}= & \left(\lambda_{2}-\lambda_{1}\right) ; \\
B_{22}= & r(k-1)+\lambda_{2}+\left(\lambda_{2}-\lambda_{1}\right)\left(p_{11}^{1}-p_{11}^{2}\right) ; \\
\Delta= & A_{12} B_{22}-A_{22} B_{12} \cdot
\end{aligned}
$$

Se $\mathrm{n}_{1}>\mathrm{n}_{2}$, a expressão mais conveniente para $t_{i}$, segundo os autores é:

$$
\hat{t}_{i}=\frac{k}{\Delta}\left[B_{21} Q_{i}-B_{11} S_{2}\left(Q_{i}\right)\right],
$$

onde

$S_{2}\left(Q_{i}\right)$ é a soma dos $Q^{\prime} s$ correspondentes aos $n_{2}$ tratamen tos que são segundos associados do i-ésimo trata mento;

$$
\begin{aligned}
A_{11} & =r(k-1)+\lambda_{1} ; \\
A_{21} & =\left(\lambda_{1}-\lambda_{2}\right) p_{12}^{1} ; \\
B_{11} & =\lambda_{1}-\lambda_{2} ; \\
B_{21} & =r(k-1)+\lambda_{1}+\left(\lambda_{1}-\lambda_{2}\right)\left(p_{22}^{2}-p_{22}^{1}\right) ; \\
\Delta & =A_{11} B_{21}-A_{21} B_{11} .
\end{aligned}
$$


Os efeitos de tratamentos são estimados por RAO (1947) usando expressões semelhantes às apresentadas por BOSE e NAIR (1939). A diferença está na definição de $S_{j}\left(Q_{j}\right)(i=1,2, \ldots, v ; j=1,2, \ldots, m)$, que para o primeiro autor é a soma dos Q's para o i-ésimo tratamento e seus j-ésimos associados. Devido a esse fato hã uma ligeira modificação na expressão de $\overleftarrow{t}_{i}$, que passa a ser

$$
\hat{t}_{i}=\frac{k}{\Delta}\left[\left(B_{22}+B_{12}\right) Q_{i}-B_{12} \Sigma Q_{i 1}\right] \text {, }
$$

para o caso onde $n_{1} \leq n_{2}$, e

$$
\hat{t}_{i}=\frac{k}{\Delta}\left[\left(B_{21}+B_{11}\right) Q_{i}-B_{11} \Sigma Q_{i 2}\right],
$$

quando $\mathrm{n}_{1} \geq \mathrm{n}_{2}$.

As expressões para a estimação dos efeitos de tratamentos, no caso de delineamentos com três classes de associados $(m=3)$, são também apresentadas por BOSE e NAIR (19.39) e RAO (1947). No caso geral, ou seja, para delineamentos com m classes de associados, CHAKRABARTI(1962) considera a seguinte igualdade:

$$
r(k-1) E_{s}=k Q_{s}+\sum_{j=1}^{m} S_{j}\left(Q_{s}\right) \sum_{i=1}^{m} \lambda_{i} f_{i j},
$$

onde $E_{s}$ é o efeito estimado do s-ésimo tratamento, $s_{j}\left(Q_{s}\right)$ é a soma dos $Q$ 's correspondentes aos $n_{j}$ tratamentos que são 
j-ésimos associados do s-ésimo tratamento, e $f_{i j}$ satisfaz a expressão

$S_{i}\left(E_{S}\right)=f_{i 1} S_{1}\left(Q_{S}\right)+f_{i_{2}} S_{2}\left(Q_{S}\right)+\ldots+f_{i m} S_{m}\left(Q_{S}\right), \quad i=1,2, \ldots, m$.

2.2.5 - Variância da estimativa de um contraste entre duas médias de tratamentos

BOSE e NAIR (1939) relatam que se $\sigma^{2}$ é a variância por parcela, então a variância da diferença entre os efeitos estimados de dois tratamentos, $E_{i}$ e $\bar{t}_{j}$, nos delineamentos com duas classes de associados, é dada por

$$
V\left(E_{i}-E_{j}\right)=2 k B_{2 I} \sigma^{2} / \Delta
$$

quando $\bar{E}_{i}$ e $\hat{t}_{j}$ são primeiros âssociados; e

$$
V\left(E_{i}-E_{j}\right)=2 \mathrm{kB}_{22} \sigma^{2} / \Delta
$$

quanto $\bar{t}_{i}$ e $\bar{t}_{j}$ são segundos associados. Segundo os autores há $\mathrm{vn}_{1} / 2$ comparações do primeiro tipo e $\mathrm{vn}_{2} / 2$ do segundo tipo. A partir deste fato apresentam a variāncia média de todas as comparações como sendo

$$
V_{m}=\frac{2 k \sigma^{2}\left[(v-1) B_{21}+n_{2} B_{11}\right]}{(v-1) \Delta}
$$


A eficiência dos delineamentos PBIB com duas classes de associados, em relação a blocos casualizados ê definida por BOSE e NAIR (1939) como sendo

$$
E=\frac{(v-1) \Delta}{r k\left[(v-1) B_{21}+n_{2} B_{11}\right]}
$$

Considerando o caso geral de delineamentos com m classes de associados, CHAKRABARTI (1962) menciona que se $t_{s}-t_{S}$ ', a diferença entre os efeitos de dois tratamentos, é estimāvel, e se o s-ésimo e o s'-ésimo tratamentos são i-ésimos associados, o melhor estimador de $t_{s}$ $t_{s}$, é $E_{s}-E_{s}$. com variância

$$
V\left(E_{S}-E_{S^{\prime}}\right)=\frac{2 \sigma^{2}}{r(k-1)}\left(k-\sum_{\ell=1}^{m} \lambda_{\ell} f_{\ell i}\right) \text {. }
$$

Pode-se observar então que os contrastes ele mentares, nos delineamentos PBIB, não são estimados com a mesma variância. 
2.3 - Ensaios em Blocos Incompletos com Alguns Tratamentos Adicionais em Cada Bloco

$$
\text { KÄLIN (1966) e FERREIRA (1980) consideram O }
$$

caso onde em cada bloco de um delineamento BIB, com parâme tros $v, b, r, k e \lambda$, são adicionados c tratamentos testemu nhas, o que resulta num total de tratamentos igual a $v^{\prime}=v+c$, e blocos de tamanho igual a $k^{\prime}=k+c$.

Os efeitos dos tratamentos são estimados por KALIN (1966) através dos trēs métodos de análise: intrablo cos, interblocós e combinação dos anteriores. Para o caso da análise intrablocos, o autor apresenta os seguintes estimadores:

i) Tratamentos adicionais:

$$
E_{a_{s^{\prime}}}=\frac{1}{b} T_{a_{s^{\prime}}}-\bar{\mu},
$$

onde, $\mathrm{T}_{\mathrm{a}_{\mathrm{S}}}$ é o total observado do tratamento adicional s'

$$
\begin{aligned}
& \left(s^{\prime}=1, \ldots, c\right) ; \\
& \hat{\mu}=\left(\sum_{s^{\prime}=1}^{c} T_{a} !\right. \\
& s !
\end{aligned}
$$
tal observado do tratamento original $s \quad(s=1, \ldots$ $\cdots, v)$. 
ii) Tratamentos do delineamento original (tratamentos originais):

$$
E_{S}=\frac{T_{S}}{r}-\hat{\mu}+\frac{(r-\lambda)\left(T_{S}-\bar{T}_{.}\right)-r\left(A_{S}-\bar{A}{ }_{0}\right)}{r(\lambda v+r C)}
$$

onde, $E_{s}$ é o efeito estimado do tratamento original s ( $s=1$,

$$
\begin{aligned}
&2, \ldots, v) ; \\
& \bar{T} .= \frac{1}{v}\left(T_{1}+T_{2}+\ldots+T_{v}\right) ; \\
& A_{S} \text { é o total dos blocos onde ocorre o tratamento or } \underline{i} \\
& \quad \text { ginal s; } \\
& \bar{A}= \frac{1}{v}\left(A_{1}+A_{2}+\ldots+A_{v}\right) .
\end{aligned}
$$

A soma de quadrados de tratamentos, ajustada para os efeitos de blocos é dada por:

$$
\text { SQTaj. = SQTad. + SQTor. (aj.) + SQTipos, }
$$

onde,

SQTad. é a soma de quadrados dos tratamentos adicionais;

SQTor.(aj.) é a soma de quadrados dos tratamentos originais ajustada; e

SQTipos é a soma de quadrados entre os dois tipos de tratamentos. 
O autor apresenta as seguintes expressões pa ra essas somas de quadrados:

i) Soma de quadrados dos tratamentos adicionais:

$$
\operatorname{sQTad}=\frac{1}{b} \sum_{s^{\prime}=1}^{c}\left(Q_{a_{s^{\prime}}}-\bar{Q}_{a}\right)^{2},
$$

onde,

$\mathrm{Q}_{\mathrm{a}_{s^{\prime}}}=\mathrm{T}_{\mathrm{a}_{\mathrm{s}^{\prime}}}-\frac{\mathrm{A}_{\mathrm{s}^{\prime}}}{\mathrm{k}^{\prime}} ; \mathrm{e}$

$\mathrm{T}_{\mathrm{a}_{\mathrm{s}^{\prime}}}$ ë o total do tratamento adicional $\mathrm{s}^{\prime}\left(\mathrm{s}^{\prime}=1, \ldots, \mathrm{c}\right)$; $A_{S}$ ' é o total dos blocos onde ocorre o tratamento adicional s':

$\bar{Q}_{a}=\frac{1}{c}\left(Q_{a_{1}}+\cdots+Q_{a_{c}}\right)$.

ii) Soma de quadrados dos tratamentos originais ajustą da:

$$
\operatorname{SoTor} \cdot(a j \cdot)=\frac{k^{\prime}}{\lambda v+r c} \sum_{s=1}^{v}\left(Q_{s}-\bar{Q}\right)^{2},
$$

onde,

$$
\begin{gathered}
Q_{S}=T_{S}-\frac{A_{S}}{k^{\prime}} ; \\
\bar{Q}=\frac{1}{v} \sum_{s=1}^{v} Q_{S} \cdot
\end{gathered}
$$


iii) Soma de quadrados entre tipos de tratamentos

$$
\text { SQTipos }=\frac{c k}{n}\left(\frac{1}{c} \sum_{s^{\prime}=1}^{c} Q_{a_{s^{\prime}}}-\frac{1}{k} \sum_{s=1}^{v} Q_{s^{\prime}}\right)^{2},
$$

onde $\mathrm{n}$ é o total de observações.

Com respeito à variância da diferença entre duas médias de tratamentos, KALIN (1966) chega aos seguintes resultados:

i) Contraste envolvendo dois tratamentos originais:

$$
\operatorname{Var}\left(\hat{\mathrm{m}}_{\mathrm{S}}-\hat{\mathrm{m}}_{\mathrm{S}^{*}}\right)=\frac{2 \mathrm{k}^{\prime}}{\lambda \mathrm{v}+\mathrm{rc}} \sigma^{2}
$$

ii) Contraste envolvendo dois tratamentos adicionais:

$$
\operatorname{Var}\left(\hat{m}_{a_{S^{\prime}}}-\hat{m}_{a_{S^{\prime} * *}}\right)=\frac{2 \sigma^{2}}{b}
$$

iii) Contraste envolvendo um tratamento original e outro adicional:

$$
\operatorname{Var}\left(\hat{m}_{s^{-}}-\hat{m}_{a_{s^{\prime}}}\right)=\left(\frac{k^{\prime}}{r v c}+\frac{c-1}{b c}+\frac{k^{\prime}(v-1)}{v(\lambda v+r c)}\right) \sigma^{2}
$$

Por outro lado, FERREIRA (1980) apresenta os seguintes estimadores para os efeitos de tratamentos: 


$$
\begin{aligned}
E_{a_{s^{\prime}}}= & \frac{1}{b} Q_{a_{s^{\prime}}} ; \text { para os tratamentos adicionais; } e \\
E_{s}= & \frac{k^{\prime}}{\lambda v+r c}\left(Q_{s}-\frac{\left(r^{2}-\lambda b\right)}{n r} \sum_{S=1}^{v} Q_{s}\right) \text {, para os tratamen } \\
& \text { tos originais. }
\end{aligned}
$$

As somas de quadrados são obtidas por:

$$
\begin{aligned}
& \text { SQTad. }=\frac{1}{b} \sum_{s^{\prime}=1}^{c} Q_{a_{s^{\prime}}}^{2}-\frac{1}{b c}\left(\sum_{s^{\prime}=1}^{c} Q_{a_{s^{\prime}}}\right)^{2},
\end{aligned}
$$

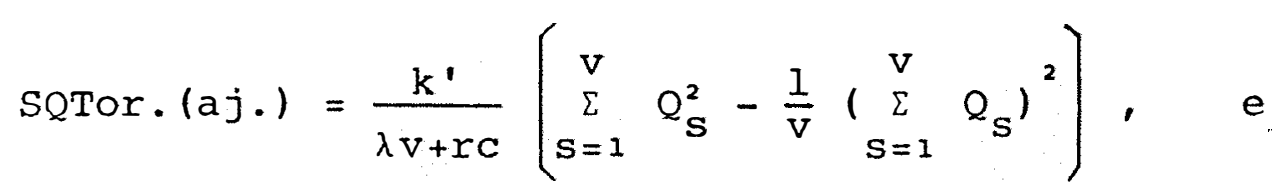

$$
\begin{aligned}
& \text { SQTipos }=\frac{k^{\prime}}{\operatorname{rvc}}\left(\sum_{i=1}^{v} Q_{s}\right)^{2} .
\end{aligned}
$$

Quanto à variância entre duas médias de tratamentos, o autor apresenta os seguintes resultados:

$$
\begin{aligned}
& \operatorname{Var}\left(\hat{\mathrm{m}}_{\mathrm{s}^{-}} \hat{\mathrm{m}}_{\mathrm{s}^{*}}\right)=\frac{2 \mathrm{k}^{\prime}}{\lambda \mathrm{v}+\mathrm{rc}} \sigma^{2}, \\
& \operatorname{Var}\left(\hat{\mathrm{m}}_{\mathrm{a}_{\mathrm{s}^{\prime}}}-\hat{\mathrm{m}}_{\left.\mathrm{a}_{\mathrm{s}^{\prime}}\right)}\right)=\frac{2}{\mathrm{~b}} \sigma^{2}, \\
& \operatorname{Var}\left(\hat{\mathrm{m}}_{\mathrm{s}^{-}} \hat{\mathrm{m}}_{\mathrm{a}_{\mathrm{s}}}\right)=\left(\frac{\mathrm{k}^{\prime}}{\lambda \mathrm{v}+\mathrm{rc}}+\frac{1}{\mathrm{~b}}-\frac{\mathrm{k}^{\prime}}{\mathrm{n}(\lambda \mathrm{v}+\mathrm{rc})} \frac{\left(\mathrm{r}^{2}-\lambda \mathrm{b}\right)}{\mathrm{r}}\right) \sigma^{2} .
\end{aligned}
$$


Pode-se observar que KÄLIN (1966) e FERREIRA (1980) apresentam estimadores diferentes para os efeitos de tratamentos. Isto deve-se ao fato dos autores não usarem a mesma restrição durante o processo de estimação. KALIN (1966) usou a restrição

$$
\underset{s^{\prime}=1}{c} E_{a_{s^{\prime}}}+\sum_{s=1}^{v} E_{s}=0,
$$

obtendo, para a méaiia geral, o estimador:

$$
\hat{\mu}=\left(\sum_{s^{\prime}=1}^{c} T_{a_{S^{\prime}}} / b+\sum_{S=1}^{v} T_{s} / r\right) /(c+v),
$$

enquanto FERREIRA (1980) considerou que:

$$
\text { b } \sum_{s^{\prime}=1}^{c} E_{a_{s^{\prime}}}+r \underset{s=1}{v} E_{s}=0,
$$

e obteve, para á média geral, o estịmador $\widehat{\mu}=G / n$, onde. $G$ é o total geral.

Com respeito às somas de quadrados e às variâncias da diferença entre duas médias, as expressões apresentadas pelos dois autores são idênticas.

Convém ressaltar que não se encontrou na literatura consultada métodos de análises, para o tipo de en saio aqui discutido, quando se consideram delineamentos em blocos incompletos parcialmente balanceados (PBIB). 
41 .

3. DESENVOLVIMENTO TEÓRICO

3.1 - Introdução

o desenvolvimento da metodologia baseia-se em um experimento, em blocos incompletos parcialmente balanceados $(\mathrm{PBIB})$, com alguns tratamentos comuns adicionados em cada bloco.

Seja, inicialmente, um experimento em blocos incompletos parcialmente balanceados, com os seguintes parâmetros :

v: número de tratamentos,

$\mathrm{b}$ : número de blocos,

$r$ : número de repetições para cada tratamento,

k: nümero de parcelas por bloco; 
e ainda $\lambda_{1}, \lambda_{2}, \ldots, \lambda_{m}, n_{1}, n_{2}, \ldots, n_{m}, p_{j k}^{i}(i, j, k=1,2$, $\ldots, m)$, definidos conforme BOSE e NAIR (1939).

Considerando-se que c novos tratamentos são adicionados a cada bloco do experimento inicial, o delinea mento resultante tem então os parâmetros:

$$
\begin{aligned}
& \mathrm{v}^{\prime}=\mathrm{v}+\mathrm{C} \text { (número de tratamentos), } \\
& \mathrm{b} \text { : número de blocos, } \\
& \mathrm{k}^{\prime}=\mathrm{k}+\mathrm{c} \text { (número de parcelas por bloco); } \\
& \mathrm{k}^{\prime} \mathrm{b}=\mathrm{n} \text { (número total de parcelas), }
\end{aligned}
$$

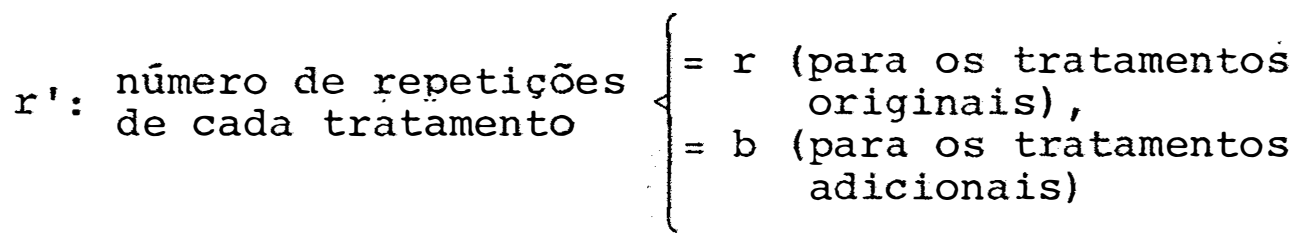

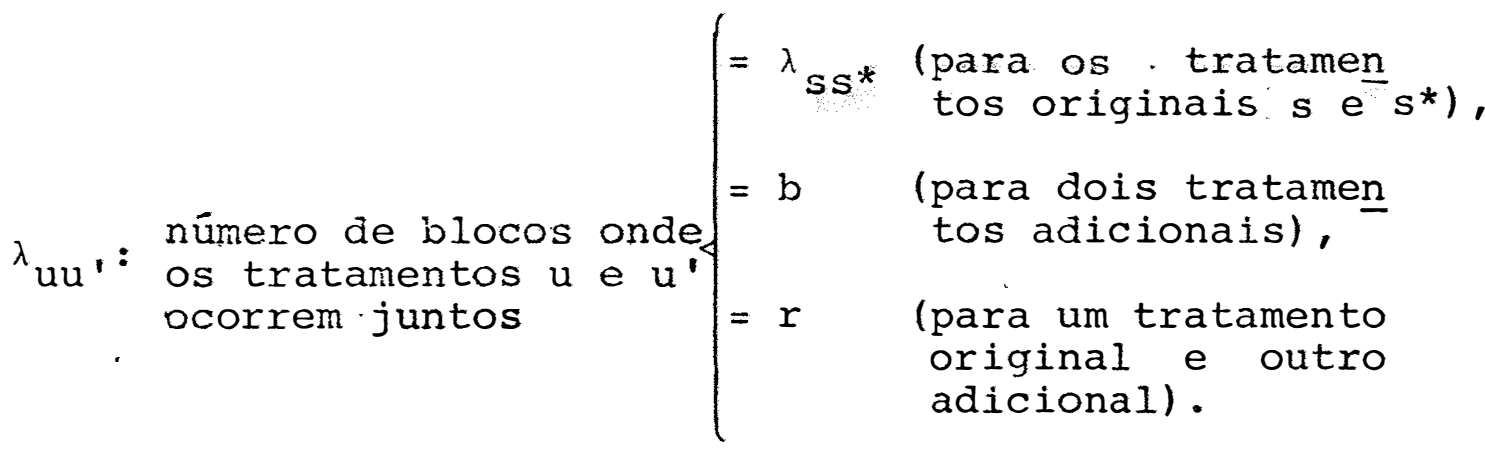

3.2 - Modelo Matemático

Para $\circ$ delineamento em questão supõe--se $\circ$ mo delo linear de efeito fixo:

$$
y_{u h}=m+t_{u}+b_{h}+e_{u h}
$$


onde,

$Y_{u h}$ é a observação do u-ésimo tratamento no h-ésimo bloco;

m è a média geral;

$t_{u}$ é o efeito do u-ésimo tratamento $\left(u=1,2, \ldots, v^{\prime}\right)$;

$b_{h}$ é o efeito do h-ésimo bloco $(h=1,2 \ldots, b)$;

e uh é o erro experimental associado à observação $Y_{\text {uh }}$ ' onde se supõe que os $\epsilon_{\text {uh }}$ 's são independentes e normalmente distribuídos, com média zero e variância $0^{2}$.

O efeito $t_{u}$ envolve $t_{s}(s=1,2, \ldots, v)$, e $t_{a_{s^{\prime}}}\left(s^{\prime}=1,2, \ldots, c\right)$, que são os efeitos dos tratamentos originais e adicionais, respectivamente.

O modelo é considerado com a restrição paramétrica:

$$
r \sum_{s=1}^{v} t_{s}+b \sum_{s^{\prime}=1}^{c} t_{a_{s^{\prime}}}=0
$$

Usando-se a forma matricial, o modelo (3.2.a)

fica :

$$
\underline{\mathrm{y}}=\underline{\mathrm{X}} \underline{\theta}+\underline{\varepsilon},
$$

onde,

y é o vetor de realizações de variáveis aleatórias;

$X$ é a matriz dos coeficientes dos parâmetros;

$\theta$ è o vetor dos parâmetros;

$\varepsilon$ é o vetor dos erros experimentais, sendo que

$$
\varepsilon \sim \mathrm{N}\left(\phi \sigma^{2} I\right) .
$$


Efetuando-se convenientemente a partição da matriz $\mathrm{x}$ obtém-se

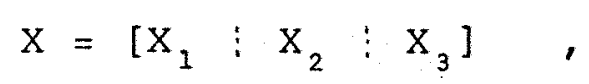

onde,

$x_{1}$ é o vetor de dimensões $\left(b k^{\prime}\right) \times(1)$, que envolve a média geral;

$\mathrm{x}_{2}$ é a matriz de dimensões (bk') $\mathrm{x}\left(\mathrm{v}^{\prime}\right)$, dos coeficientes associados aos tratamentos;

$\mathrm{x}_{3}$ é a matriz de dimensões (bk') $\mathrm{x}(\mathrm{b})$, dos coeficientes associados aos blocos.

A partição do vetor $\underset{\sim}{\theta}$, correspondente à partição da matriz $x$ é

$$
\theta^{\prime}=\left[m: \underline{\sim}^{\prime}: \underline{\sim}^{\prime}\right],
$$

onde,

m é a média geral;

I é o vetor dos efeitos dos tratamentos, de dimensões ( $\left.v^{\prime}\right) \times(1)$;

$\underset{\sim}{\beta}$ é o vetor dos efeitos dos blocos, de dimensões (b) $\mathrm{x}(1)$.

Pode-se ainda considerar-se a partição

$$
\underline{\tau}^{\prime}=\left[\begin{array}{l:ll}
\tau & 1 & \tau_{2}^{\prime}
\end{array}\right],
$$


onde,

I 1 é o vetor dos efeitos dos tratamentos originais, de dimensões (v) x (1);

$I_{\sim}$ é o vetor dos efeitos dos tratamentos adicionais, de dimensões (c) $x$ (1).

A restrição paramétrica, nesse caso, é $\mathrm{x}_{1}^{\prime} \mathrm{x}_{2} \tau=\underset{\sim}{\phi}$

3.3 - Sistema de Equações Normais

O sistema de equações normais, obtido através do método dos quadrados minimos, é:

$$
x^{\prime} x_{\sim}^{\hat{\theta}}=x^{\prime} \underline{\sim},
$$

ou seja,

$$
\left(\begin{array}{ccc}
x_{1}^{\prime} x_{1} & x_{1}^{\prime} x_{2} & x_{1}^{\prime} x_{3} \\
x_{2}^{\prime} x_{1} & x_{2}^{\prime} x_{2} & x_{2}^{\prime} x_{3} \\
x_{3}^{\prime} x_{1} & x_{3}^{\prime} x_{2} & x_{3}^{\prime} x_{3}
\end{array}\right)\left(\begin{array}{c}
\hat{m} \\
\tilde{\tau} \\
\tilde{\tilde{\beta}}
\end{array}\right)=\left(\begin{array}{c}
x_{1}^{\prime} \underset{\sim}{y} \\
x_{2}^{\prime} \underset{\sim}{y} \\
x_{3}^{\prime} \underset{\sim}{y}
\end{array}\right)
$$

As matrizes do sistema anterior são designa-

das por:

$$
\begin{aligned}
& \mathrm{x}_{1}^{\prime} \mathrm{x}_{1}=\mathrm{bk}^{\prime}=\mathrm{n}=\text { número total de unidades experimentais; } \\
& x_{1}^{\prime} x_{2}={ }_{1}^{x_{v^{\prime}}^{\prime}}=\left(\begin{array}{c:c}
x_{1}^{\prime} & \vdots \\
\sim^{\prime} & x_{c}^{\prime}
\end{array}\right)=[r, r, \ldots, r \vdots b, b, \ldots, b] \\
& \text { (vetor associado aos números de repetições dos tra } \\
& \text { tamentos originais e adicionais); }
\end{aligned}
$$




$$
x_{1}^{\prime} x_{3}={ }_{1} \underset{\sim}{z} b^{\prime}=\left[k^{\prime}, k^{\prime}, \ldots, k^{\prime}\right] \text { (vetor associado ao nú- }
$$
mero de unidades experimentais por blocol;

$x_{2}^{\prime} x_{2}=v^{\prime} R_{v^{\prime}}=\left(\begin{array}{ll}R_{1} & \phi \\ \phi & R_{2}\end{array}\right)$ (matriz das repetições dos tra tamentos), onde $R_{1}$ é a matriz das repetições dos tratamentos originais, e $R_{2}$ a matriz das repeti ções dos tratamentos adicionais;

$\mathrm{x}_{2}^{\prime} \mathrm{x}_{3}={ }_{\mathrm{v}} \mathrm{N}_{\mathrm{b}}=\left[\begin{array}{c}\mathrm{N}_{1} \\ \mathrm{~N}_{2}\end{array}\right) \quad$ (matriz de incidência dos tratamentos em relação aos blocos); onde $\mathrm{N}_{1}$ refere-se aos tratamentos originais, $\mathrm{e}_{2}$ aos tratamentos adicionais. Fazendo $N=\left[n_{u h}\right]$, então $n_{u h}$ é o número de vezes que o u-ésimo tratamento ocorre no h-ésimo bloco;

$\begin{aligned} \mathrm{X}_{3}^{\prime} \mathrm{X}_{3}= & \mathrm{b}^{K_{\mathrm{b}}}=\mathrm{k}^{\prime} \cdot \mathrm{I}_{\mathrm{b}} \quad \text { (matriz associada ao número de unida } \\ & \text { des experimentais por bloco). }\end{aligned}$

Assim, o sistema de equações em [3.3.b] pode ser escrito na forma:

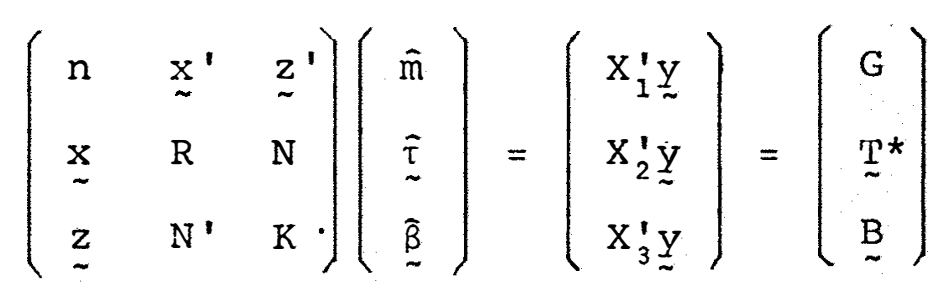


onde,

$$
\begin{aligned}
& \hat{\mathrm{m}}=\text { efeito estimado da média geral; }
\end{aligned}
$$

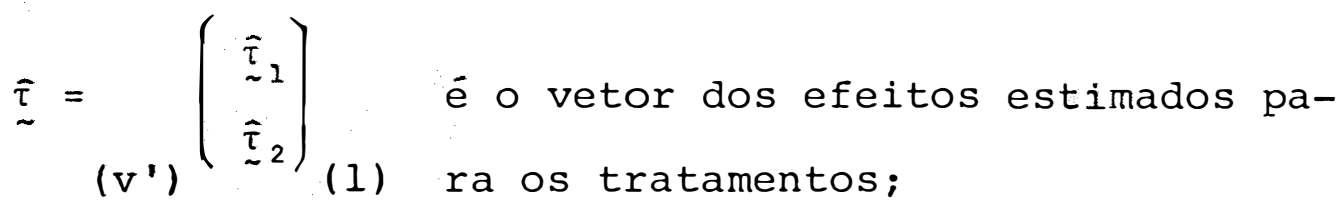

$$
\begin{aligned}
& \underline{\sim}_{\text {(v) }}=\left(\begin{array}{c}
E_{1} \\
E_{2} \\
\cdots \\
E_{v}
\end{array}\right)_{(1)} \\
& \tilde{\tau}_{2}=\left(\begin{array}{c}
E_{a_{1}} \\
E_{a_{2}} \\
\cdots \\
E_{a_{c}}
\end{array}\right)_{(1)} \text { é o vetor dos efeitos estimados pa- } \\
& \left.\underset{\widehat{B}}{\stackrel{(b)}{\sigma_{b}}}\right]_{\text {(1) }} \quad \begin{array}{c}
\bar{b}_{1} \\
\sigma_{2} \\
\cdots
\end{array} \text { ra blocos; } \\
& \text { é o vetor dos efeitos estimados pa- }
\end{aligned}
$$




$$
\begin{aligned}
& {\stackrel{\sim}{T^{*}}=}_{\left(V^{\prime}\right)}\left(\begin{array}{c}
T_{\sim}^{*} \\
T_{2}^{*}
\end{array}\right) \text { (1) tratamentos; } \\
& \underset{\sim}{\mathrm{T}_{1}^{*}=}\left(\begin{array}{c}
\mathrm{T}_{1} \\
\mathrm{~T}_{2} \\
\cdots \\
\mathrm{T}_{\mathrm{V}}
\end{array}\right)_{\text {(1) }} \text { tratamentos originais; }
\end{aligned}
$$$$
\underset{\sim}{\mathrm{T}_{2}^{*}}=\left(\begin{array}{c}
\mathrm{T}_{\mathrm{a}_{1}} \\
\mathrm{~T}_{\mathrm{a}_{2}} \\
\cdots \\
\mathrm{T}_{\mathrm{a}_{\mathrm{C}}}
\end{array} \text { (1) }_{\text {tratamentos adicionais; }}\right. \text { o vetor dos totais observados dos }
$$$$
\underset{\sim}{B}=\left(\begin{array}{c}
B_{1} \\
B_{2} \\
\cdots \\
B_{b}
\end{array} \text { (b) }_{\text {é o vetor dos totais observados pa- }}\right. \text { ra blocos. }
$$ 
3.4 - Estimação do Efeito da Média Geral

$$
\text { O sistema [3.3.c] resulta nas seguintes equa }
$$

ఢ̧ões :

$$
\begin{aligned}
& \mathrm{bk}^{\prime} \overline{\mathrm{m}}+\mathrm{x}^{\prime} \underset{\sim}{\bar{\tau}}+\underset{\sim}{z^{\prime}}{\underset{\sim}{\hat{B}}}^{-}=\mathrm{G} \\
& \underset{\sim}{\mathrm{x}} \hat{\mathrm{m}}+\mathrm{R}_{\sim}^{\hat{\tau}}+\underset{\sim}{\mathrm{N}} \hat{\tilde{\beta}}={\underset{\sim}{T}}^{*} \quad[3.4 . \mathrm{a}] \\
& \underset{\sim}{2 \hat{m}}+N^{\prime} \underline{\underline{\tau}}+K \underset{\sim}{\hat{B}}=\underset{\sim}{B}
\end{aligned}
$$

Impondo-se as restrições usuais,

$$
r \underset{s=1}{v} E_{s}+b \sum_{s^{\prime}=1}^{t} E_{a_{s^{\prime}}}=0 ; \sum_{h=1}^{b} \hat{b}_{h}=0[3.4 . b]
$$

ou seja, $\underset{\sim}{x}{ }_{\sim}^{\hat{\tau}}=0$ e ${\underset{\sim}{z}}^{\prime} \underset{\sim}{\hat{B}}=0$, resulta que

$$
\begin{aligned}
& b k^{\prime} \text { 角 } \quad=G \\
& \underset{\sim}{\hat{m}}+R_{\sim}^{\bar{\tau}}+N \underline{\tilde{B}}=\stackrel{T}{T}^{*} \\
& \underset{\sim}{\underline{m}}+N^{\prime} \hat{\underline{\tau}}+\underset{\sim}{\hat{\beta}}=\underline{\sim}
\end{aligned}
$$

Da primeira equação obtém-se

$$
\hat{\mathrm{m}}=\frac{\mathrm{G}}{\mathrm{bk}}
$$

e os efeitos de $\underset{\sim}{\hat{\tau}}$ e $\underset{\sim}{\hat{\beta}}$ são obtidos de

$$
\begin{aligned}
& \mathrm{R} \hat{\tilde{\tau}}+\underset{\sim}{N \hat{\beta}}=\underline{\sim}^{*}-\underset{\sim}{x} \hat{m} \cdot[a] ， \\
& \mathrm{~N}^{\prime} \underset{\sim}{\underline{\tau}}+\underset{\sim}{\hat{\beta}}=\underset{\sim}{\mathrm{B}}-\underset{\sim}{z} \hat{\mathrm{m}} \quad[\mathrm{b}],
\end{aligned}
$$


ou seja, de:

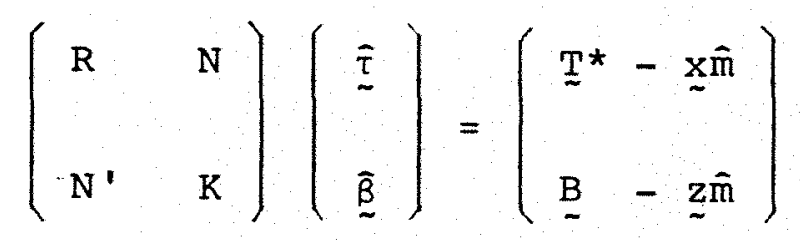

3.5 - Estimação dos Efeitos de Tratamentos Ajustados para Blocos

Para estimarem-se os efeitos de tratamentos, ajustados para blocos, os efeitos de blocos devem ser eliminados da equação [a] em [3.4.e]. Com esse objetivo adiciona-se à equação [a] a equação [b], pré-multiplicada por $\left(-\mathrm{NK}^{-1}\right)$. Isso resulta em:

$$
\left(R-\frac{1}{k^{\prime}} N^{\prime}\right) \underline{\tau}=\underline{\sim}^{*}-\frac{1}{k^{\prime}} N \underline{-},
$$

que equivale a pré-multiplicar o sistema [3.4.f] pela matriz $w$, onde

$$
W=\left[I_{v},-N K^{-1}\right]
$$

\subsection{1 - Método clássico}

O sistema de equações em [3.5.a] pode ser es crito na forma

$$
C \bar{\tau}=\underline{Q},
$$

onde c é uma matriz simétrica, de característica (v'-l) e 
e dimensões $\left(v^{\prime}\right) \times\left(v^{\prime}\right), \tilde{\tau}$ é um vetor $\left(v^{\prime}\right) \times(l)$ e $Q$ é um vetor $\left(v^{\prime}\right) x(1)$, denominado de vetor dos totais ajustados dos trata mentos. Dado um tratamento u qualquer, então o elemento de Q correspondente a u, é a diferença entre o total do trata mento u e o total dos blocos, onde u ocorre, dividido por k'. Os elementos de C, em correspondência com os tratamentos, são:

$$
\begin{aligned}
& c_{s s}=\frac{r\left(k^{\prime}-1\right)}{k^{\prime}} \text {, para os tratamentos originais; } \\
& c_{s s^{*}}=c_{s^{*} s}=-\frac{\lambda_{i j}}{k^{\prime}} \text {, para quaisquer dois tratamen- } \\
& \text { tos originais, ou ainda, } \\
& c_{s s^{*}}=-\frac{\lambda_{l}}{k^{\prime}} \text {, para quaisquer dois tratamentos originais } \\
& \text { que são l-ésimos associados; } \\
& c_{s^{\prime} s^{\prime}}=\frac{b(b-l)}{k^{\prime}} \text {, para os tratamentos adicionais; }
\end{aligned}
$$

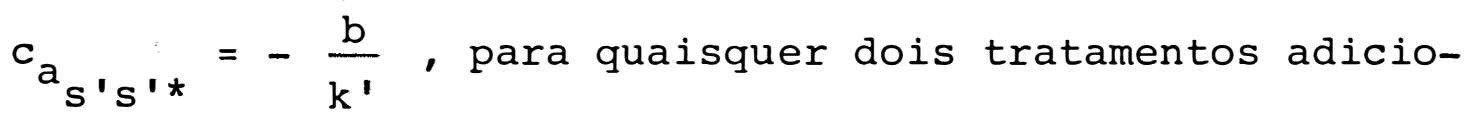

$$
\begin{aligned}
& \text { nais: } \\
& c_{s s^{\prime}}=-\frac{r}{k^{\prime}} \text {, para um tratamento original e outro adi- } \\
& \text { cional. }
\end{aligned}
$$


Como C é uma matriz singular, o sistema [3.5.1.a] pode ser resolvido, conforme PAVATE (1961) e PIMENTEL GOMES (1967) dentre outros, mediante a introdução de uma matriz A, com as mesmas dimensões de $\mathrm{C}$, denominada "matriz de restri ção", tal que a solução do sistema seja única. As restrições introduzidas produzem um novo sistema de equações, que pode ser escrito na forma:

$$
\mathrm{A}_{\tilde{\tau}}^{\hat{\tau}}=\underline{\underline{\phi}}
$$

A matriz A, para o caso em questão, geralmen te tem a seguinte estrutura:

$$
A=\left(-\frac{r}{k^{\prime}}\left(v^{\prime}\right)^{E}(v)-\frac{b}{k^{\prime}}\left(v^{\prime}\right)^{E}(c)\right) \quad[3.5 .1 . c]
$$

onde $\mathrm{E}$ é uma matriz de uns.

Pode-se também tomar uma matriz de restrição simétrica substituindo-se A por A'A.

De [3.5.1.a] e [3.5.1.b] obtém-se:

$$
(C-A) \underset{\sim}{\bar{\tau}}=\stackrel{Q}{Q},
$$

onde $C-A=M$ é uma matriz não singular. Logo, a solução do sistema é dada por:

$$
\tilde{\tau}=M^{-1} \underline{\sim} \quad
$$




\subsection{2 - Método simplificado}

Considerando-se as expressões das diferentes matrizes em [3.3], o sistema de equações em [3.5.a] pode ser colocado na seguinte forma:

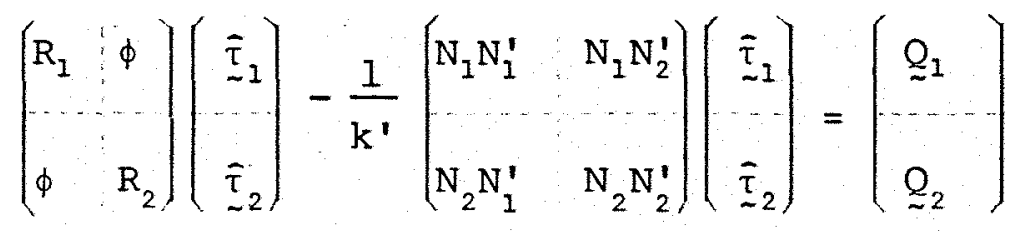

onde ${\underset{\sim}{1}}_{1}$ é $O$ vetor dos totais ajustados para os tratamentos originais, e $\underline{\sim}_{2} \circ$ vetor dos totais ajustados para os tratamentos adicionais.

Baseando-se nas partições das matrizes do sị tema anterior, obtêm-se as expressões dos estimadores dos efeitos de tratamentos.

$$
\begin{aligned}
3.5 .2 .1 & \text { Estimação dos efeitos dos trata } \\
& \text { mentos adicionais }
\end{aligned}
$$

Do sistema [3.5.2.a] tem-se que:

$$
R_{2} \tilde{\tau}_{\sim 2}-\frac{1}{k^{\prime}}\left(N_{2} N_{1}^{\prime} \bar{\tau}_{\sim 1}+N_{2} N_{2}^{\prime} \bar{\tau}_{2}=Q_{\sim} \quad\right. \text { [3.5.2.1.a] }
$$

Como cada tratamento adicional ocorre sempre uma vez em cada bloco segue-se que:

$$
\mathrm{N}_{2}=\mathrm{c}_{\mathrm{b}}^{\mathrm{E}_{\mathrm{b}}} \text {, onde } \mathrm{E} \text { é uma matriz de uns; e ainda, }
$$




$$
\begin{aligned}
& N_{2} N_{1}^{\prime}=c_{b} E_{b}^{\left(N_{1}^{\prime}\right)} v=r c_{v}^{E_{v}} \quad[3.5 .2 .1 . b] \\
& \mathrm{N}_{2} \mathrm{~N}_{2}^{\prime}=c^{E_{b}} \quad b^{E_{c}}=b \quad c_{c}^{E_{c}} \quad[3.5 .2 .1 . c]
\end{aligned}
$$

Logo, o sistema [3.5.2.1.a] fica:

$$
R_{2} \bar{\tau}_{2}-\frac{1}{k^{\prime}}\left(r c^{E} v \bar{\tau}_{1}+b c^{E} c \bar{\tau}_{2}\right)=Q_{2} .
$$

Retomando-se a restrição $r_{c}{ }^{E} \bar{\tau}_{1}+b{ }_{c} \bar{\tau}_{c}{\underset{\sim}{\tau}}_{2}=0$, tem-se então que:

$$
\bar{\tau}_{2}=\frac{1}{b} \underline{Q}_{2},
$$

visto que $R_{2}$ é uma matriz diagonal com todos os elementos iguais a b.

$$
\text { Para um tratamento particular s', segue-se }
$$

que:

$$
E_{a_{s^{\prime}}}=\frac{1}{b} Q_{a_{s^{\prime}}}, \quad[3.5 \cdot 2 \cdot 1 . e]
$$

onde $Q_{a_{s^{\prime}}}=T_{a_{s^{\prime}}}-\frac{G}{k^{\prime}}$ e $s^{\prime}=1,2, \ldots, c$. 


\subsubsection{2 - Estimação dos efeitos dos trata- mentos originais com $m$ classes de associados}

O sistema de equações referente aos tratamen tos originais, obtido de [3.5.2.a], é:

$$
R_{1} \bar{\tau}_{1}-\frac{1}{k^{\prime}} N_{1} N_{1}^{\prime} \tilde{\tau}_{1}-\frac{1}{k^{\prime}} N_{1} N_{2}^{\prime} \bar{\tau}_{2}=Q_{1} .
$$

Para um dado tratamento s pode-se verificar

que:

$$
Q_{s}=r\left(1-\frac{1}{k^{\prime}}\right) E_{s}-\frac{1}{k^{\prime}}\left(\begin{array}{l}
\lambda_{s 1} E_{1}+\ldots+\lambda_{s s-1} E_{s-1}+ \\
+\lambda_{s s+1} E_{s+1}+\ldots+\lambda_{s v} E_{v}
\end{array}\right)-\frac{r}{k^{\prime}} \sum_{s^{\prime}=1}^{c} E_{a_{s^{\prime}}} E^{\prime}
$$

$s=1,2, \ldots, v$; ou ainda,

$$
k^{\prime} Q_{s}=r\left(k^{\prime}-1\right) E_{s}-\sum_{i=1}^{m} \lambda_{i} s_{i}\left(E_{s}\right)-r \sum_{s^{\prime}=1}^{c} E_{a_{s} \prime^{\prime}} \text { [3.5.2:2.a] }
$$

onde $Q_{S}=T_{S}-A_{S} / K^{\prime}$, sendo $A_{S}$ o total dos blocos onde ocor re o tratamento original $s, \lambda_{i}(i=1,2, \ldots, m)$ é um dos parâme tros do primeiro tipo, definido por BOSE e NAIR (1939), e $S_{i}\left(E_{s}\right)$ representa a soma dos i-ésimos associados de $E_{S}$. De [3.5.2.1.e] tem-se que:

$$
\sum_{s^{\prime}=1}^{c} E_{a_{s^{\prime}}}=\frac{1}{b} \sum_{s^{\prime}=1}^{c} O_{a_{s^{\prime}}}
$$


logo, a expressão [3.5.2.2.a] fica:

$$
k^{\prime} Q_{s}+\frac{r}{b} \sum_{s^{\prime}=1}^{c} Q_{a_{s^{\prime}}}=r\left(k^{\prime}-1\right) E_{s}-\sum_{i=1}^{m} \lambda_{i} s_{i}\left(E_{s^{\prime}}\right)[3 \cdot 5 \cdot 2 \cdot 2 . c]
$$

Como pode-se observar, o efeito de $E_{S}$ na for ma em que é colocado, é uma função de seus associados, que também não são conhecidos. Assim, é necessārio que a expressão [3.5.2.2.c] seja modificada.

Sabe-se por CHAKRABARTI (1962), que se um con junto de tratamentos obedece à estrutura de um delineamento $\mathrm{PBIB}$, as seguintes igualdades se verificam:

$$
s_{j} s_{i}\left(E_{s}\right)=\left\{\begin{array}{l}
\sum_{j=1}^{m} p_{j ' i}^{j} s_{j}\left(E_{s}\right), \text { se } j^{\prime} \neq i, \text { e } \\
n_{i} E_{s}+\sum_{j=1}^{m} p_{i i}^{j} s_{j}\left(E_{s}\right), \text { se } j^{\prime}=i,
\end{array}\right.
$$

onde os parâmetros $n_{i}$ e $p_{i j}^{k}(i, j . k=1,2, \ldots, m)$ são definidos conforme BOSE e NAIR (1939).

Usando-se o mesmo procedimento de CHAKRABARTI (1962), para o problema aqui discutido, tem-se então que :

$$
S_{j^{\prime}}\left(k^{\prime} Q_{S}+\frac{r}{b} \sum_{S^{\prime}=1}^{c} Q_{a_{S^{\prime}}}\right)=r\left(k^{\prime}-1\right) s_{j^{\prime}}\left(E_{S^{\prime}}\right)-\sum_{i=1}^{m} \lambda_{i^{\prime}} S_{j^{\prime}} S_{i}\left(E_{S^{\prime}}\right)
$$


Como $\frac{r}{b} \sum_{s^{\prime}=1}^{c} Q_{a_{s}}$, é uma constante, a expressão anterior fica:

$$
k^{\prime} S_{j} \cdot\left(Q_{s}\right)+n_{j} \cdot \frac{r}{b} \sum_{s^{\prime}=1}^{c} Q_{a_{s^{\prime}}}=r\left(k^{\prime}-1\right) S_{j},\left(E_{s}\right)-\sum_{i=1}^{m} \lambda_{i} S_{j} s_{i}\left(E_{s^{\prime}}\right),
$$

onde $S_{j} \cdot\left(Q_{s}\right)$ representa a soma dos $Q$ 's dos j'-ésimos associados do s-ésimo tratamento. Logo, tem-se:

a) $\quad k^{\prime} S_{1}\left(Q_{s}\right)+n_{1} \frac{r}{b} \sum_{s^{\prime}=1}^{c} Q_{a_{s^{\prime}}}=r\left(k^{\prime}-1\right) S_{1}\left(E_{s}\right)-\sum_{i=1}^{m} \lambda_{i} S_{1} S_{i}\left(E_{s^{\prime}}\right)$,

$\operatorname{para} j^{\prime}=1 ;$

onde,

$$
\begin{aligned}
\sum_{i=1}^{m} \lambda_{i} S_{1} S_{i}\left(E_{S}\right) & =\lambda_{1} S_{1} S_{1}\left(E_{s}\right)+\lambda_{2} S_{1} S_{2}\left(E_{S}\right)+\ldots+\lambda_{m} S_{1} S_{m}\left(E_{S}\right) \\
& =\lambda_{1}\left[n_{1} E_{S}+p_{11}^{1} S_{1}\left(E_{S}\right)+p_{11}^{2} S_{2}\left(E_{s}\right)+\ldots+p_{11}^{m} S_{m}\left(E_{S}\right)\right]+ \\
& +\lambda_{2}\left[p_{12}^{1} S_{1}\left(E_{s}\right)+p_{12}^{2} S_{2}\left(E_{S}\right)+\ldots+p_{12}^{m} S_{m}\left(E_{S}\right)\right]+\ldots+ \\
& +\lambda_{m}\left[p_{1 m}^{1} S_{1}\left(E_{S}\right)+p_{1 m_{2}}^{2} S_{2}\left(E_{S}\right)+\ldots+p_{1 m}^{m} S_{m}\left(E_{S}\right)\right]
\end{aligned}
$$

Sabe-se ainda por BOSE e NAIR (1939), CHAKRA BARTI (1962), dentre outros, que: 


$$
\begin{aligned}
\sum_{j^{\prime}=1}^{m} p_{j j^{\prime}}^{i} & =n_{j}-1, \text { se } j=i, \\
& =n_{j}, \text { se } j \neq i,
\end{aligned}
$$

Considerando-se essas igualdades em [3.5.2.2.g], segue-se que:

$$
\begin{aligned}
& \sum_{i=1}^{m} \lambda_{i} s_{1} s_{i}\left(E_{s}\right)=\lambda_{1}\left(\begin{array}{l}
n_{1} E_{s}+\left(n_{1}-1-p_{12}^{1}-\ldots-p_{1 m}^{1}\right) s_{1}\left(E_{s}\right)+ \\
+\left(n_{1}-p_{12}^{2}-\ldots-p_{1 m}^{2}\right) s_{2}\left(E_{s}\right)+\ldots+ \\
+\left(n_{1}-p_{12}^{m}-\ldots-p_{1 m}^{m}\right) s_{m}\left(E_{s}\right)
\end{array}\right)+ \\
& +\lambda_{2}\left[p_{12}^{1} S_{1}\left(E_{s}\right)+p_{12}^{2} S_{2}\left(E_{s}\right)+\ldots+p_{12}^{m} S_{m}\left(E_{s}\right)\right]+\ldots+ \\
& +\lambda_{m}\left[p_{1 m}^{1} s_{1}\left(E_{s}\right)+p_{1 m_{2}}^{2} s_{s}\left(E_{s}\right)+\ldots+p_{1 m}^{m} s_{m}\left(E_{s}\right)\right] \\
& =\lambda_{1}\left(\begin{array}{l}
n_{1} E_{s}+n_{1} S_{1}\left(E_{s}\right)+n_{1} S_{2}\left(E_{s}\right)+\ldots+n_{1} s_{m}\left(E_{s}\right)- \\
-\left(1+p_{12}^{1}+\ldots+p_{1 m}^{1}\right) s_{1}\left(E_{s}\right)- \\
-\left(p_{12}^{2}+\ldots+p_{1 m}^{2}\right) s_{2}\left(E_{s}\right)-\ldots- \\
-\left(p_{12}^{m}+\ldots+p_{1 m}^{m}\right) s_{m}\left(E_{s}\right)
\end{array}\right]+ \\
& +\lambda_{2}\left[p_{12}^{1} s_{1}\left(E_{s}\right)+p_{12}^{2} s_{2}\left(E_{s}\right)+\ldots+p_{12}^{m} s_{m}\left(E_{s}\right)\right]+\ldots+ \\
& +\lambda_{m}\left[p_{1 m_{1}}^{1} s_{s}\left(E_{s}\right)+p_{1 m}^{2} s_{2}\left(E_{s}\right)+\ldots+p_{1 m}^{m} s_{m}\left(E_{s}\right)\right] \\
& \text { Mas, } n_{1} E_{s}+n_{1} s_{1}\left(E_{s}\right)+\ldots+n_{1} s_{m}\left(E_{s}\right)=n_{1} \sum_{s=1}^{v} E_{s} ; \\
& \text { e por [3.4.b] verifica-se que } \sum_{s=1}^{v} E_{s}=-\frac{b}{r} \sum_{s^{\prime}=1}^{c} E_{a_{s^{\prime}}} \text {. }
\end{aligned}
$$


Como $\sum_{s^{\prime}=1}^{c} E_{a_{s^{\prime}}}=\frac{1}{b} \sum_{s^{\prime}=1}^{c} o_{a_{s^{\prime}}}$, por [3.5.2.2.b], então, $\sum_{s=1}^{v} E_{s}=-\frac{1}{r}{\underset{s}{s^{\prime}=1}}_{\sum_{s^{\prime}}} Q_{a_{s^{\prime}}}$. Portanto, segue-se que:

$$
\begin{aligned}
\sum_{i=1}^{m} \lambda_{i} S_{1} S_{i}\left(E_{s}\right)= & -\frac{\lambda_{1} n_{1}}{r} \sum_{s^{\prime}=1}^{c} Q_{s^{\prime}}-\lambda_{1}\left[\begin{array}{l}
\left(n_{1}-p_{11}^{1}\right) s_{1}\left(E_{s}\right)+ \\
+\left(n_{1}-p_{11}^{2}\right) S_{2}\left(E_{s}\right)+\ldots+ \\
+\left(n_{1}-p_{11}^{m}\right) S_{m}\left(E_{s}\right)
\end{array}\right]+ \\
& +\lambda_{2}\left[p_{12}^{1} S_{1}\left(E_{s}\right)+\underline{p}_{12}^{2} S_{2}\left(E_{s}\right)+\ldots+p_{12}^{m} S_{m}\left(E_{s}\right)\right]+\ldots+ \\
& +\lambda_{m}\left[p_{1 m}^{1} S_{1}\left(E_{s}\right)+p_{1 m}^{2} S_{2}\left(E_{s}\right)+\ldots+p_{1 m}^{m} S_{m}\left(E_{s}\right)\right],
\end{aligned}
$$

ou seja,

$$
\begin{aligned}
\sum_{i=1}^{m} \lambda_{i} S_{1} S_{i}\left(E_{s}\right)= & -\frac{\lambda_{1} n_{1}}{r} \sum_{s^{\prime}=1}^{c} o_{S_{s^{\prime}}}-\left(\begin{array}{c}
\lambda_{1} n_{1}-\lambda_{1} p_{11}^{1}-\lambda_{2} p_{12}^{1}- \\
-\ldots-\lambda_{m} p_{1 m}^{1}
\end{array}\right) s_{1}\left(E_{s}\right)- \\
& -\left[\lambda_{1} n_{1}-\lambda_{1} p_{11}^{2}-\lambda_{2} p_{12}^{2}-\ldots-\lambda_{m} p_{1 m}^{2}\right] s_{2}\left(E_{s}\right)-\ldots- \\
& -\left[\lambda_{1} n_{1}-\lambda_{1} p_{11}^{m}-\lambda_{2} p_{12}^{m}-\ldots-\lambda_{m} p_{1 m}^{m}\right] S_{m}\left(E_{s}\right)
\end{aligned}
$$

Voltando em [3.5.2.2.f], e considerando-se a expressão [3.5.2.2.i], segue-se que: 


$$
\begin{aligned}
& k^{\prime} S_{1}\left(Q_{s}\right)+\left(\frac{r^{2}-\lambda_{1} b}{b r}\right) n_{1} \sum_{s^{\prime}=1}^{c} Q_{a_{s^{\prime}}}=\left(r\left(k^{\prime}-1\right)+\lambda_{1} n_{1}-\sum_{\ell=1}^{m} \lambda_{\ell} p_{1 \ell}^{1}\right) S_{1}\left(E_{s^{\prime}}\right)+ \\
& +\left(\lambda_{1} \mathrm{n}_{1}-\sum_{\ell=1}^{m} \lambda_{\ell} \mathrm{p}_{1 \ell}^{2}\right) \mathrm{S}_{2}\left(E_{S}\right)+\ldots+ \\
& +\left(\lambda_{1} n_{1}-\sum_{l=1}^{m} \lambda_{\ell} p_{1 \ell}^{m}\right) S_{m}\left(E_{s}\right)
\end{aligned}
$$

b) $\quad k^{\prime} S_{2}\left(Q_{s}\right)+n_{2} \frac{r}{b} \sum_{s^{\prime}=1}^{c} Q_{a_{s^{\prime}}}=r\left(k^{\prime}-1\right) s_{2}\left(E_{s}\right)-\sum_{i=1}^{m} \lambda_{1} S_{2} s_{i}\left(E_{s^{\prime}}\right)$, para $j !=2 ;$

onde,

$$
\begin{aligned}
\sum_{i=1}^{m} \lambda_{i} S_{2} S_{i}\left(E_{s}\right)= & \lambda_{1} S_{2} S_{1}\left(E_{S}\right)+\lambda_{2} S_{2} S_{2}\left(E_{s}\right)+\ldots+\lambda_{m} S_{2} S_{m}\left(E_{S}\right) \\
= & \lambda_{1}\left[p_{21}^{1} S_{1}\left(E_{s}\right)+p_{21}^{2} S_{2}\left(E_{s}\right)+\ldots+p_{21}^{m} S_{m}\left(E_{S}\right)\right]+ \\
& +\lambda_{2}\left[n_{2} E_{S}+p_{22}^{1} S_{1}\left(E_{s}\right)+p_{22}^{2} S_{2}\left(E_{S}\right)+\ldots+p_{22}^{m} S_{m}\left(E_{s}\right)\right]+\ldots+ \\
& +\lambda_{m}\left[p_{2 m}^{1} S_{1}\left(E_{S}\right)+p_{2 m}^{2} S_{2}\left(E_{s}\right)+\ldots+p_{2 m}^{m} S_{m}\left(E_{s}\right)\right]
\end{aligned}
$$


De $[3.5 .2 .2 . h]$ segue-se que:

$$
\begin{aligned}
& \sum_{i=1}^{m} \lambda_{i} S_{2} S_{1}\left(E_{s}\right)=\lambda_{1}\left[p_{21}^{1} S_{1}\left(E_{S}\right)+p_{21}^{2} S_{2}\left(E_{s}\right)+\ldots+p_{21}^{m} S_{m}\left(E_{s}\right)\right]+ \\
& +\lambda_{2}\left(\begin{array}{l}
n_{2} E_{s}+\left(n_{2}-p_{21}^{1}-p_{23}^{1}-\ldots-p_{2 m}^{1}\right) s_{1}\left(E_{s}\right)+ \\
+\left(n_{2}-1-p_{21}^{2}-p_{23}^{2}-\ldots-p_{2 m}^{2}\right) s_{2}\left(E_{s}\right)+\ldots+ \\
+\left(n_{2}-p_{21}^{m}-p_{23}^{m}-\ldots-p_{2 m}^{m}\right) s_{m}\left(E_{s}\right)
\end{array}\right) \\
& \left.+\ldots+\lambda_{m}\left[p_{2 m}^{1} S_{1}\left(E_{s}\right)+p_{2 m_{2}^{2}}^{2} S_{S}\right)+\ldots+p_{2 m}^{m} S_{m}\left(E_{s}\right)\right] \\
& =\lambda_{1}\left[p_{21}^{1} S_{1}\left(E_{S}\right)+p_{21}^{2} S_{2}\left(E_{S}\right)+\ldots+p_{21}^{m} S_{m}\left(E_{S}\right)\right]+ \\
& +\lambda_{2}\left(\begin{array}{l}
n_{2} E_{S}+n_{2} S_{1}\left(E_{S}\right)+n_{2} S_{2}\left(E_{S}\right)+\ldots+ \\
+n_{2} S_{m}\left(E_{S}\right)-\left(p_{21}^{1}+p_{23}^{2}+\ldots+p_{2 m}^{1}\right) S_{1}\left(E_{S}\right)- \\
-\left(1+p_{2 I}^{2}+p_{23}^{2}+\ldots+p_{2 m}^{2}\right) S_{2}\left(E_{S}\right)-\ldots- \\
-\left(p_{21}^{m}+p_{23}^{m}+\ldots+p_{2 m}^{m}\right) S_{m}\left(E_{S}\right)
\end{array}\right)+\ldots+ \\
& +\lambda_{m}\left[p_{2 m}^{1} S_{1}\left(E_{S}\right)+p_{2 m}^{2} S_{2}\left(E_{S}\right)+\ldots+p_{2 m}^{m} S_{m}\left(E_{s}\right)\right] \\
& =-\frac{\lambda_{2} n_{2}}{r} \sum_{s^{\prime}=1}^{c} Q_{a_{S^{\prime}}}+\lambda_{1}\left(\begin{array}{l}
p_{21}^{1} S_{1}\left(E_{s}\right)+p_{21}^{2} s_{2}\left(E_{s}\right)+ \\
+\ldots+p_{21}^{m} S_{m}\left(E_{s}\right)
\end{array}\right)- \\
& -\lambda_{2}\left(\begin{array}{l}
\left(n_{2}-p_{22}^{1}\right) s_{1}\left(E_{S}\right)+\left(n_{2}-p_{22}^{2}\right) s_{2}\left(E_{s}\right)+ \\
+\ldots+\left(n_{2}-p_{22}^{m}\right) S_{m}\left(E_{s}\right)
\end{array}\right)+\ldots+ \\
& +\lambda_{m}\left[P_{2 m}^{1} S_{1}\left(E_{S}\right)+p_{2 m}^{2} S_{2}\left(E_{S}\right)+\ldots+p_{2 m}^{m} S_{m}\left(E_{S}\right)\right] \text {. }
\end{aligned}
$$




$$
\begin{aligned}
\sum_{i=1}^{m} \lambda_{i} S_{2} S_{i}\left(E_{S}\right)= & -\frac{\lambda_{2} n_{2}}{r} \sum_{S^{\prime=1}}^{c} Q_{s^{\prime}}-\left(\begin{array}{l}
\lambda_{2} n_{2}-\lambda_{1} p_{21}^{1}-\lambda_{2} p_{22}^{1}- \\
-\ldots-\lambda_{m} p_{2 m}^{1}
\end{array}\right) S_{1}\left(E_{s}\right)- \\
& -\left[\lambda_{2} n_{2}-\lambda_{1} p_{21}^{2}-\lambda_{2} p_{22}^{2}-\ldots-\lambda_{m} p_{2 m}^{2}\right] S_{2}\left(E_{s}\right)-\ldots- \\
& -\left[\lambda_{2} n_{2}-\lambda_{1} p_{21}^{m}-\lambda_{2} p_{22}^{m}-\ldots-\lambda_{m} p_{2 m}^{m}\right] S_{m}\left(E_{s}\right)
\end{aligned}
$$

Voltando em [3.5.2.2.k], e considerando-se $[3.5 .2 .2 . \mathrm{m}]$, segue-se que:

$$
\begin{aligned}
& k^{\prime} S_{2}\left(Q_{s}\right)+\left(\frac{r^{2}-\lambda_{2} b}{b r}\right) n_{2} \sum_{S^{\prime}=1}^{c} Q_{a_{s^{\prime}}}=\left(\lambda_{2} n_{2}-\sum_{\ell=1}^{m} \lambda_{\ell} p_{2 \ell}^{1}\right) S_{1}\left(E_{s^{\prime}}\right)+ \\
& +\left(r\left(k^{\prime}-1\right)+\lambda_{2} n_{2}-\sum_{\ell=1}^{m} \lambda_{\ell} p_{2 \ell}^{2}\right) S_{2}\left(E_{s}\right)+ \\
& +\ldots+\left(\lambda_{2} n_{2}-\sum_{\ell=1}^{m} \lambda_{\ell} p_{2 \ell}^{m}\right) S_{m}\left(E_{s}\right) \\
& \text { [3.5.2.2.n] }
\end{aligned}
$$

c) $\quad k^{\prime} s_{m}\left(Q_{s}\right)+n_{m} \frac{r}{b} \sum_{s^{\prime}=1}^{c} Q_{a_{s^{\prime}}}=r(k-I) s_{m}\left(E_{s^{\prime}}\right)-\sum_{i=1}^{m} \lambda_{i} S_{m} s_{i}\left(E_{s^{\prime}}\right)$,

$\operatorname{para} j^{\prime}=m$;

onde, 


$$
\begin{aligned}
& \text { m } \\
& \sum_{i=1} \lambda_{i} S_{m} S_{i}\left(E_{s}\right)=\lambda_{1} S_{m} S_{1}\left(E_{s}\right)+\lambda_{2} S_{m} S_{2}\left(E_{s}\right)+\ldots+\lambda_{m} S_{m} S_{m}\left(E_{s}\right) \\
& =\lambda_{1}\left[p_{m_{1}}^{1} S_{1}\left(E_{s}\right)+p_{m I}^{2} S_{2}\left(E_{s}\right)+\ldots+p_{m 1}^{m} S_{m}\left(E_{s}\right)\right]+ \\
& +\lambda_{2}\left[p_{m_{2}}^{1} S_{1}\left(E_{s}\right)+p_{m 2}^{2} S_{2}\left(E_{s}\right)+\ldots+p_{m 2}^{m} S_{m}\left(E_{s}\right)\right]+\ldots+ \\
& +\lambda_{m}\left[n_{m} E_{s}+p_{m m_{1}}^{1} s_{s}\left(E_{s}\right)+p_{m m}^{2} s_{2}\left(E_{s}\right)+\ldots+p_{m m}^{m} s_{m}\left(E_{s}\right)\right] \\
& =\lambda_{1}\left[p_{m_{1}}^{1} S_{1}\left(E_{s}\right)+p_{m_{1}}^{2} S_{2}\left(E_{s}\right)+\ldots+p_{m_{1}}^{m} S_{m}\left(E_{s}\right)\right]+ \\
& +\lambda_{2}\left[p_{m 2}^{1} s_{1}\left(E_{s}\right)+p_{m 2}^{2} s_{2}\left(E_{s}\right)+\ldots+p_{m 2}^{m} s_{m}\left(E_{s}\right)\right]+\ldots+ \\
& +\lambda_{m}\left(\begin{array}{l}
n_{m} E_{s}+\left(n_{m}-p_{m 1}^{1}-p_{m 2}^{1}-\ldots-p_{m(m-1)}^{1}\right) s_{1}\left(E_{s}\right)+ \\
+\left(n_{m}-p_{m 1}^{2}-p_{m 2}^{2}-\ldots-p_{m(m-1)}^{2}\right) s_{2}\left(E_{s}\right)+\ldots+ \\
+\left(n_{m}-1-p_{m 1}^{m}-p_{m 2}^{m}-\ldots-p_{m(m-1)}^{m}\right) s_{m}\left(E_{s}\right)
\end{array}\right) \\
& =\lambda_{1}\left[p_{m 1}^{1} s_{1}\left(E_{s}\right)+p_{m_{1}}^{2} s_{2}\left(E_{s}\right)+\ldots+p_{m 1}^{m} s_{m}\left(E_{s}\right)\right]+ \\
& +\lambda_{2}\left[p_{m 2}^{1} s_{1}\left(E_{s}\right)+p_{m 2}^{2} s_{2}\left(E_{s}\right)+\ldots+p_{m 2}^{m} s_{m}\left(E_{s}\right)\right]+\ldots+ \\
& +\lambda_{m}\left(\begin{array}{l}
n_{m} E_{s}+n_{m} s_{1}\left(E_{s}\right)+n_{m} s_{2}\left(E_{s}\right)+\ldots+n_{m} s_{m}\left(E_{s}\right)- \\
-\left(p_{m 1}^{1}+p_{m 2}^{1}+\ldots+p_{m(m-1)}^{1}\right) s_{1}\left(E_{s}\right)- \\
\left.-\left(p_{m 1}^{2}+p_{m 2}^{2}+\ldots+p_{m(m-1)}^{2}\right) s_{2}\left(E_{s}\right)-\ldots-p_{m(m-1)}\right) s_{m}\left(E_{s}\right) \\
-\left(1+p_{m 1}^{m}+p_{m 2}^{m}+\ldots+p_{m}^{m}\right.
\end{array}\right)
\end{aligned}
$$




$$
\begin{aligned}
& \sum_{i=1}^{m} \lambda_{i} S_{m} S_{i}\left(E_{s}\right)=-\frac{\lambda_{m} m_{m}}{r} \sum_{s^{\prime}=1}^{c} Q_{a_{s^{\prime}}}+\lambda_{1}\left(\begin{array}{l}
p_{m 1}^{1} s_{1}\left(E_{s}\right)+p_{m 1}^{2} s_{2}\left(E_{s}\right)+ \\
+\ldots+p_{m l}^{m} s_{m}\left(E_{s}\right)
\end{array}\right)+ \\
& +\lambda_{2}\left[p_{m 2}^{1} s_{1}\left(E_{s}\right)+p_{m 2}^{2} s_{2}\left(E_{s}\right)+\ldots+p_{m 2}^{m} s_{m}\left(E_{s}\right)\right]-\ldots- \\
& -\lambda_{m}\left(\begin{array}{l}
\left(n_{m}-p_{m m}^{1}\right) s_{1}\left(E_{s}\right)+\left(n_{m}-p_{m m}^{2}\right) s_{2}\left(E_{s}\right)+ \\
+\cdots+\left(n_{m}-p_{m m}^{m}\right) s_{m}\left(E_{s}\right)
\end{array}\right) \\
& =-\frac{\lambda_{m} n_{m}}{r} \sum_{s^{\prime}=1}^{c} Q_{a_{s}}-\left(\begin{array}{l}
\lambda_{m} n_{m}-\lambda_{1} p_{m 1}^{1}-\lambda_{2} p_{m 2}^{1} \\
-\cdots-\lambda_{m} p_{m}^{1}
\end{array}\right) S_{1}\left(E_{s}\right)- \\
& -\left[\lambda_{m} n_{m}-\lambda_{1} p_{m 1}^{2}-\lambda_{2} p_{m 2}^{2}-\ldots-\lambda_{m} p_{m m}^{2}\right] S_{2}\left(E_{s}\right)-\ldots- \\
& -\left[\lambda_{m} n_{m}-\lambda_{1} p_{m 1}^{m}-\lambda_{2} p_{m 2}^{m}-\ldots-\lambda_{m} p_{m m}^{m}\right] S_{m}\left(E_{s}\right)
\end{aligned}
$$

Voltando em [3.5.2.2.o], e considerando-se [3.5.2.2.p], segue-se que:

$$
\begin{aligned}
& k^{\prime} S_{m}\left(Q_{s}\right)+\left(\frac{r^{2}-\lambda_{m}^{b}}{b r}\right) n_{m} \sum_{s^{\prime}=1}^{c} Q_{a_{s^{\prime}}}=\left[\lambda_{m} n_{m}-\sum_{\ell=1}^{m} \lambda_{\ell} p_{m \ell}^{1}\right] S_{1}\left(E_{s}\right)+ \\
& +\left[\lambda_{m} n_{m}-\sum_{\ell=1}^{m} \lambda_{\ell} p_{m}^{2} l^{2} S_{2}\left(E_{S}\right)+\ldots+\right. \\
& +\left[r\left(k^{\prime}-1\right)+\lambda_{m} n_{m}-\sum_{\ell=1}^{m} \lambda_{\ell} p_{m_{\ell}}^{m}\right] s_{m}\left(E_{s}\right)
\end{aligned}
$$


Considerando-se as expressões a), b) e c) po de-se verificar por $[3.5 .2 .2 . j],[3.5 .2 .2 . n]$ e $[3.5 .2 .2 . q]$ que:

$$
\begin{aligned}
k^{\prime} S_{i}\left(Q_{s}\right)+\left(\frac{r^{2}-\lambda_{i} b}{b r}\right) n_{i} \sum_{S^{\prime}=1}^{c} Q_{a_{s^{\prime}}}= & a_{i 1} s_{1}\left(E_{s}\right)+a_{i 2} s_{2}\left(E_{s}\right)+\ldots+ \\
& +a_{i m} s_{m}\left(E_{S^{\prime}},\right.
\end{aligned}
$$

onde,

$$
\begin{aligned}
& a_{i j}=\lambda_{i} n_{i}-\sum_{\ell=1}^{m} \lambda_{\ell} p_{i \ell}^{j}, \quad i \neq j \quad \text { e } i, j=1,2, \ldots, m \\
& a_{i i}=r\left(k^{\prime}-1\right)+\lambda_{i} n_{i}-\sum_{\ell=1}^{m} \lambda_{\ell} p_{i \ell}^{i}
\end{aligned}
$$

De [3.5.2.2.r] pode-se escrever então que:

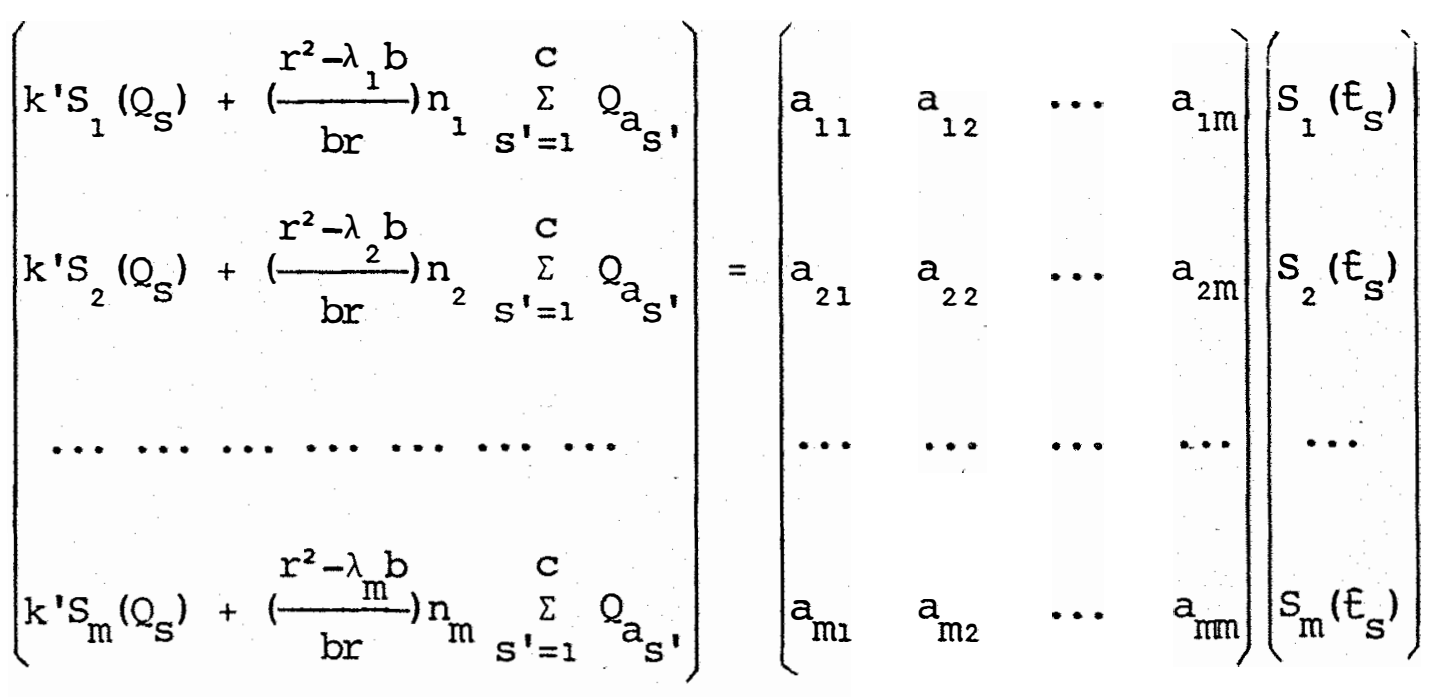


De forma análoga ao procedimento de CHAKRABARTI (1962), se U é a matriz formada pelos elementos $a_{i j}$ $(i, j=1,2, \ldots, m)$, com inversa $U^{-1}$, de elementos $f_{i j}(i, j=1$, $2, \ldots, m)$, tem-se que:

$$
\begin{aligned}
& s_{i}\left(E_{s}\right)=f_{i I}\left(k^{\prime} s_{1}\left(Q_{s}\right)+\left(\frac{r^{2}-\lambda_{1} b}{b r}\right) n_{1} \sum_{s^{\prime}=1}^{c} Q_{a_{s^{\prime}}}\right)+ \\
& +f_{i 2}\left(k^{\prime} s_{2}\left(Q_{s}\right)+\left(\frac{r^{2}-\lambda_{2} b}{b r}\right) n_{2} \sum_{s^{\prime}=1}^{c} Q_{a_{s^{\prime}}}\right)+ \\
& +\ldots+f_{i m}\left(k^{\prime} s_{m}\left(Q_{s}\right)+\left(\frac{r^{2}-\lambda_{m}^{b}}{b r}\right) n_{m} \sum_{s^{\prime}=1}^{c} Q_{a_{s^{\prime}}}\right)
\end{aligned}
$$

De $[3.5 .2 .2 . c]$, sabe-se que:

$$
r\left(k^{\prime}-1\right) E_{s}=k^{\prime} Q_{s}+\frac{r}{b} \underset{s^{\prime}=1}{c} Q_{a_{s}}+\sum_{i=1}^{m} \lambda_{i} s_{i}\left(E_{s^{\prime}}\right)
$$

Substituindo-se $S_{i}\left(E_{S}\right)$ pela expressäo encontrada em [3.5.2.2.u], segue-se então que:

$$
\begin{aligned}
& r\left(k^{\prime}-1\right) E_{s}=k^{\prime} Q_{s}+\frac{r}{b} \sum_{s^{\prime}=1}^{c} Q_{a_{s^{\prime}}}+\sum_{i=1}^{m} \lambda_{i} f_{i 1}\left(k^{\prime} S_{1}\left(Q_{s^{\prime}}\right)+\left(\frac{r^{2}-\lambda_{1} b}{b r}\right) n_{1} \sum_{s^{\prime}=1}^{c} Q_{a_{s^{\prime}}}\right. \\
& +\sum_{i=1}^{m} \lambda_{i} f_{i 2}\left[k^{\prime} s_{2}\left(Q_{s}\right)+\left(\frac{r^{2}-\lambda_{2} b}{b r}\right) n_{2} \sum_{s^{\prime}=1}^{c} Q_{s^{\prime}}\right]+\cdots+ \\
& +\sum_{i=1}^{m} \lambda_{i} f_{i m}\left(k^{\prime} s_{m}\left(Q_{s}\right)+\left(\frac{r^{2}-\lambda_{m}^{b}}{b r}\right) n_{m} \sum_{s^{\prime}=1}^{c} Q_{a_{s}}\right)
\end{aligned}
$$

e, finalmente, 


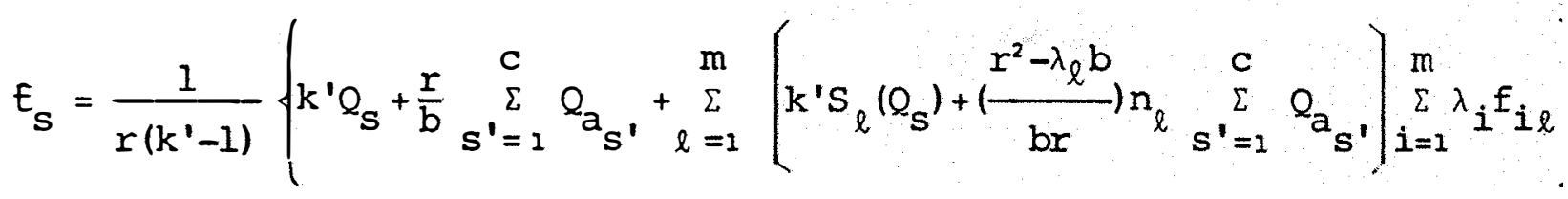

$[3.5 .2 .2 . v]$

3.5.2.3 - Estimação dos efeitos dos tratamentos originais com duas classes de associados

Os delineamentos PBIB com duas classes de as sociados, $m=2$, são de particular importância, visto que são os mais utilizados na prática, conforme relatam BOSE e NAIR (1939). Nesse caso a expressão para $E_{S}$ passa a ser:

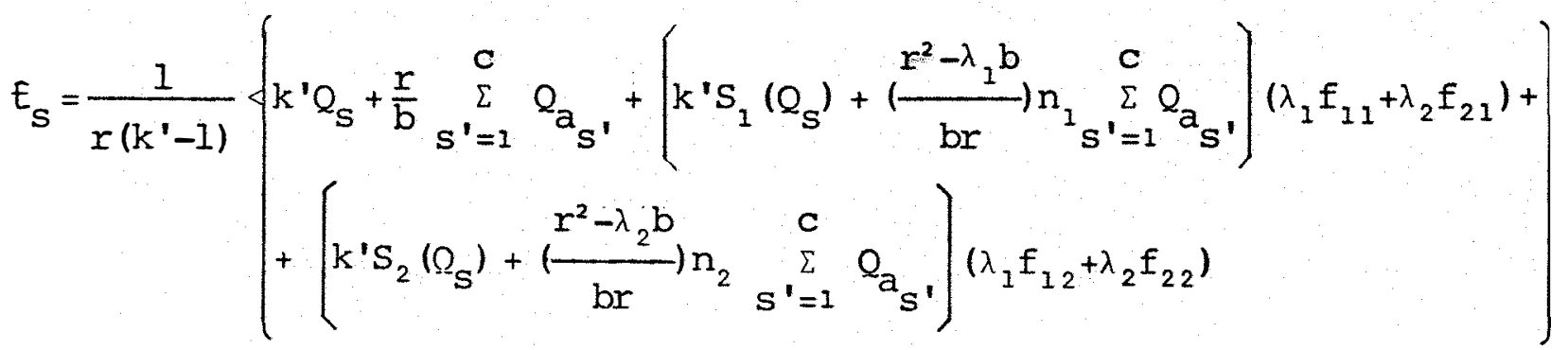
$[3 \cdot 5 \cdot 2.3 . a]$

A matriz $U$, formada pelos elementos $a_{i j}(i, j=$ 1,2), tem a forma: 


$$
U=\left(\begin{array}{ll}
a_{11} & a_{12} \\
a_{21} & a_{22}
\end{array}\right)
$$

onde, por [3.5.2.2.s],

$$
\begin{aligned}
& a_{11}=r\left(k^{\prime}-1\right)+\lambda_{1} n_{1}-\lambda_{1} p_{11}^{1}-\lambda_{2} p_{12}^{1} \\
& =r\left(k^{\prime}-1\right)+\lambda_{1}\left(n_{1}-p_{11}^{1}\right)-\lambda_{2} p_{12}^{1} \\
& =r\left(k^{\prime}-1\right)+\lambda_{1}\left(p_{12}^{1}+1\right)-\lambda_{2} p_{12}^{1} \\
& =r\left(k^{\prime}-1\right)+\left(\lambda_{1}-\lambda_{2}\right) p_{12}^{1}+\lambda_{1} \\
& a_{12}=\lambda_{1} n_{1}-\lambda_{1} p_{11}^{2}-\lambda_{2} p_{12}^{2} \\
& =\lambda_{1} n_{1}-\lambda_{1}\left(n_{1}-p_{12}^{2}\right)-\lambda_{2} p_{12}^{2} \\
& =\left(\lambda_{1}-\lambda_{2}\right) p_{12}^{2} \\
& a_{21}=\lambda_{2} n_{2}-\lambda_{1} p_{21}^{1}-\lambda_{2} p_{22}^{1} \\
& =\lambda_{2} n_{2}-\lambda_{1} p_{21}^{1}-\lambda_{2}\left(n_{2}-p_{21}^{1}\right) \\
& =\left(\lambda_{2}-\lambda_{1}\right) \mathrm{p}_{2 \cdot 1}^{1} \\
& a_{22}=r\left(k^{\prime}-1\right)+\lambda_{2} n_{2}-\lambda_{1} p_{21}^{2}-\lambda_{2} p_{22}^{2} \\
& =r\left(k^{\prime}-1\right)+\lambda_{2}\left(n_{2}-p_{22}^{2}\right)-\lambda_{1} p_{21}^{2} \\
& =r\left(k^{\prime}-1\right)+\lambda_{2}\left(p_{21}^{2}+1\right)-\lambda_{1} p_{21}^{2} \\
& =r\left(k^{\prime}-1\right)+\left(\lambda_{2}-\lambda_{1}\right) p_{21}^{2}+\lambda_{2}
\end{aligned}
$$

A matriz $U^{-1}$, com elementos $f_{i j}(i, j=1,2)$, é: 


$$
U^{-1}=\left(\begin{array}{ll}
f_{11} & f_{12} \\
f_{21} & f_{22}
\end{array}\right)
$$

onde, fazendo $\Delta=a_{11} a_{22}-a_{12} a_{21}$,

$$
f_{11}=\frac{a_{22}}{\Delta}, f_{12}=-\frac{a_{12}}{\Delta}, f_{21}=-\frac{a_{21}}{\Delta} \text { e } f_{22}=\frac{a_{11}}{\Delta}
$$

$$
\text { Assim, a expressão para } E_{S} \text { fica: }
$$

$$
E_{s}=\frac{1}{r\left(k^{\prime}-1\right)}\left\{\begin{array}{l}
k^{\prime} Q_{s}+\frac{r}{b} \sum_{s^{\prime}=1}^{c} Q_{a_{s^{\prime}}}+\frac{1}{\Delta}\left(k^{\prime} s_{1}\left(Q_{s}\right)+\left(\frac{r^{2}-\lambda_{1} b}{b r}\right) n_{1} \sum_{s^{\prime}=1}^{c} Q_{a_{s^{\prime}}}\right. \\
+\frac{1}{\Delta}\left(k^{\prime} S_{2}\left(Q_{s}\right)+\left(\frac{r^{2}-\lambda_{2} b}{b r}\right) n_{2} \sum_{s^{\prime}=1}^{c} Q_{a_{s^{\prime}}} Q_{\left(\lambda_{2} a_{11}-\lambda_{1} a_{12}\right)}\right.
\end{array}\right.
$$

Por outro lado, sabe-se que:

$$
\begin{aligned}
Q_{S}=T_{s}-\frac{A_{S}}{k^{\prime}}, & \text { onde } T_{s} \text { é o total do s-ésimo tratamento } \\
& \text { original, e } A_{S} \text { é o total dos blocos onde } \\
& \text { esse tratamento ocorre; }
\end{aligned}
$$

$Q_{a_{s^{\prime}}}=\mathrm{T}_{a_{s^{\prime}}}-\frac{G}{k^{\prime}}$, onde $T_{a_{s^{\prime}}}$ é 0 total do j-ésimo tratamen
to adicional, e G é o total geral. 
Logo,

$$
\underset{s_{s=1}}{v} Q_{s^{\prime}}+\sum_{s^{\prime}=1}^{c} Q_{a_{s^{\prime}}}=\sum_{s}^{v} T_{s}-\frac{k G}{k^{\prime}}+\sum_{s^{\prime}=1}^{c} T_{a_{s^{\prime}}}-\frac{c G}{k^{\prime}}=G-G=0,
$$

e

$$
\sum_{s^{\prime}=1}^{c} Q_{a_{s^{\prime}}}=-\sum_{s_{s=1}}^{v} Q_{s}
$$

Considerando-se a igualdade anterior, a expressão em [3.5.2.3.g] pode ser colocada na seguinte forma:

$$
E_{S}=\frac{1}{r\left(k^{\prime}-1\right) \Delta}\left\{\begin{array}{l}
k^{\prime}\left[\Delta Q_{S}+\stackrel{\star}{A_{1}} S_{1}\left(Q_{S}\right)+\AA_{2}^{*} S_{2}\left(Q_{S}\right)\right]- \\
-\frac{1}{b r}\left[\Delta r^{2}+\left(r^{2}-\lambda_{1} b\right) n_{1} A_{1}^{*}+\left(r^{2}-\lambda_{2} b\right) n_{2} \stackrel{\star}{A}_{2}^{*} \underset{S=1}{v} Q_{S}\right.
\end{array}\right\},
$$

onde $\stackrel{\star}{A_{1}}=\lambda_{1} a_{22}-\lambda_{2} a_{21}$ e $\stackrel{\star}{A_{2}}=\lambda_{2} a_{11}-\lambda_{1} a_{12}$.

$$
\text { Como } \sum_{S=1}^{V} Q_{S}=Q_{S}+S_{1}\left(Q_{S}\right)+S_{2}\left(Q_{S}\right) \text {, a expressão }
$$

[3.5.2.3.i] pode ainda ser modificada de modo que $S_{1}\left(Q_{s}\right)$ ou $S_{2}\left(Q_{S}\right)$ seja eliminado. Se $n_{1}<n_{2}$, é conveniente eliminar- se $S_{2}\left(Q_{S}\right)$, caso contrário elimina-se $S_{1}\left(Q_{S}\right)$. 
Para o primeiro caso, $\mathrm{n}_{1}<\mathrm{n}_{2}$, deve-se substituir $s_{2}\left(Q_{s}\right)$, em [3.5.2.3.i], por $\sum_{s=1} Q_{s}-Q_{s}-S_{1}\left(Q_{s}\right)$. I so resulta em:

$$
E_{S}=\frac{1}{r\left(k^{\prime}-1\right) \Delta}\left\{\begin{array}{ll}
k^{\prime}\left[\left(\Delta-\stackrel{\star}{A}_{2}\right) Q_{S}+\left(\stackrel{\star}{A}_{1}-\stackrel{A}{A}_{2}\right) S_{1}\left(Q_{S}\right)\right]- \\
-\frac{1}{b r}\left[\Delta r^{2}+\left(r^{2}-\lambda_{1} b\right) n_{1} \stackrel{\star}{A}_{1}+\left(r^{2}-\lambda_{2} b\right) n_{2} \stackrel{A}{A}_{2}-b r k^{\prime} A_{2}^{*} \underset{S=1}{v} Q_{S}\right.
\end{array}\right\}
$$

Para o segundo caso, $\mathrm{n}_{2}<\mathrm{n}_{1}$, deve-se substi tuir $s_{1}\left(Q_{s}\right)$, em [3.5.2.3.i], por $\sum_{s=1}^{v} Q_{s}-Q_{s}-s_{2}\left(Q_{s}\right)$. Isso resulta em:

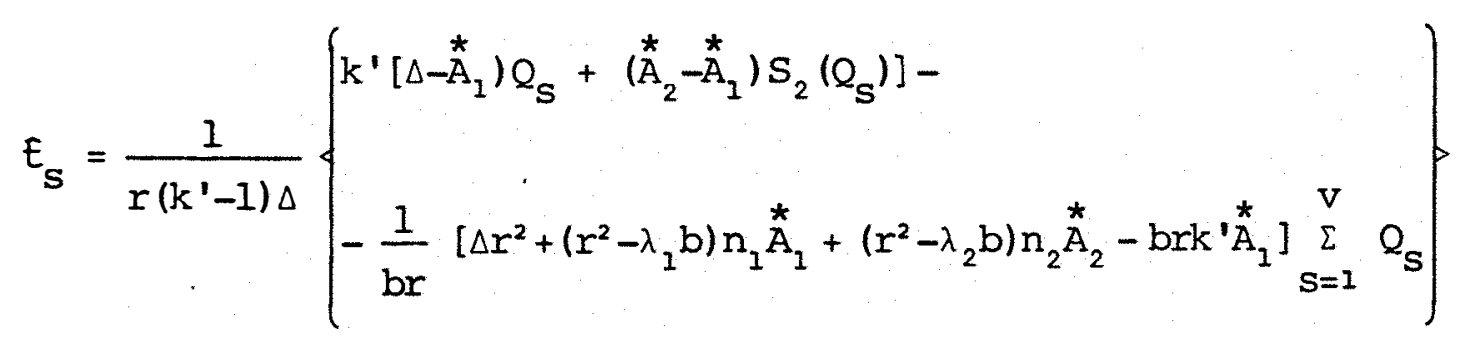




\subsubsection{4 - Caso especial: Estimação dos efei tos dos tratamentos originais em delineamento BIB}

A expressão para os efeitos dos tratamentos estimados pode ser obtida, nesse caso, a partir de um delineamento $\mathrm{PBIB}$, considerando-se $\lambda_{1}=\lambda_{2}=\ldots=\lambda_{\mathrm{m}}=\lambda$. Tomando-se como base 0 PBIB com duas classes de associados, tem-se então que:

$$
\begin{aligned}
& a_{11}=a_{22}=r\left(k^{\prime}-1\right)+\lambda ; \quad a_{12}=a_{21}=0 \text {; } \\
& \Delta=\left[r\left(k^{\prime}-1\right)+\lambda\right]^{2} ; \quad \stackrel{\star}{A}_{1}=\stackrel{\star}{A}_{2}=\left[r\left(k^{\prime}-1\right)+\lambda\right] \lambda ; \\
& \mathrm{n}_{1}+\mathrm{n}_{2}=\mathrm{v}-1 \\
& E_{s}=\frac{k^{\prime}\left[r\left(k^{\prime}-1\right)+\lambda\right]}{r\left(k^{\prime}-1\right)\left[r\left(k^{\prime}-1\right)+\lambda\right]^{2}}\left\{r\left(k^{\prime}-1\right) Q_{s}-\frac{1}{k^{\prime} b r}\left(\begin{array}{l|l}
r^{3}\left(k^{\prime}-1\right)+r^{2} \lambda+ & v \\
\left(r^{2}-\lambda b\right) \lambda(v-1)-b r k^{\prime} \lambda
\end{array}\right) Q_{s=1}^{v} Q_{S}\right\} \\
& =\frac{k^{\prime}}{r\left(k^{\prime}-1\right)+\lambda}\left\{Q_{S}-\frac{1}{r^{2}\left(k^{3}-1\right) k^{\prime} b}\left(\begin{array}{l}
r^{3} k^{\prime}-r^{3}+r^{2} \lambda+ \\
\left(r^{2}-\lambda b\right) r(k-1)-b r k^{\prime} \lambda
\end{array}\right) \sum_{s=1}^{v} Q_{s}\right\},
\end{aligned}
$$

visto que, em delineamento BIB, a igualdade $\lambda(v-1)=r(k-1)$ se verifica. Sabe-se tambëm que $k^{\prime}=k+c$ e $b k=r v ;$ logo, 
$E_{s}=\frac{k^{\prime}}{r(k-1)+r c+\lambda}\left\{Q_{s}-\frac{1}{r^{2}\left(k^{\prime}-1\right) k^{\prime} b}\left(\begin{array}{l}r^{3} k+r^{3} c-r^{3}+r^{2} \lambda+\left(r^{2}-\lambda b\right) r\left(k^{\prime}-1\right)- \\ -\left(r^{2}-\lambda b\right) r c-b r k \lambda-b r c \lambda\end{array}\right) \begin{array}{c}v \\ \sum Q_{s=1} s\end{array}\right\}$

$=\frac{k^{\prime}}{\lambda(v-1)+r c+\lambda}\left\{Q_{S}-\frac{1}{r^{2}\left(k^{\prime}-1\right) k^{\prime} b}\left(\begin{array}{l}r^{3}(k-1)+r^{3} c+r^{2} \lambda+\left(r^{2}-\lambda b\right) r\left(k^{\prime}-1\right)- \\ -r^{3} c+\lambda b r c-r^{2} \lambda v-b r c \lambda\end{array}\right) \begin{array}{l}v \\ \sum\end{array}\right.$

$\left.=-\frac{k^{\prime}}{\lambda v+r c} \int_{Q_{S}}-\frac{1}{r^{2}\left(k^{\prime}-1\right) k^{\prime} b}\left(\begin{array}{l}r^{3}(k-1)-r^{2} \lambda(v-1)+ \\ +\left(r^{2}-\lambda b\right) r\left(k^{\prime}-1\right)\end{array}\right) \begin{array}{cc}v & \\ \sum_{S=1} & Q_{S}\end{array}\right)$

- Como $r^{3}(k-1)=r^{2} \lambda(v-1)$, segue-se portanto que:

$$
E_{S}=-\frac{k^{\prime}}{\lambda v+r c}\left(Q_{S}-\frac{\left(r^{2}-\lambda b\right)}{r k^{\prime} b} \sum_{S=1}^{v} Q_{S}\right) \text {, }
$$

que é a expressão encontrada por FERREIRA (1980). 
3.6 - Propriedades das Soluções do Sistema de Equaçōes Normais com Efeitos de Blocos Eliminados

Sabe-se, por $[3.5 .1 . e]$, que $\underset{\tilde{I}}{=}=\mathrm{M}^{-1} \stackrel{\varrho}{\text {, e por }}$ [3.5.2.e], que $E_{a_{s^{\prime}}}=\frac{l}{b} Q_{a_{s^{\prime}}}$. Além disso, a expressão para $E_{S}$, em [3.5.2.2.v] pode ser colocada na forma:

$$
E_{s}=\frac{1}{r\left(k^{\prime}-1\right)}\left\{\begin{array}{l}
k^{\prime}\left(Q_{s}+\sum_{\ell=1}^{m} s_{\ell}\left(Q_{s}\right) \sum_{i=1}^{m} \lambda_{i} f_{i \ell}\right)+ \\
+\left(\frac{r}{b}+\sum_{l=1}^{m}\left(\frac{r^{2}-\lambda l^{b}}{b r}\right) n_{\ell} \underset{i=1}{m} \lambda_{i} f_{i \ell}\right) \underset{s^{\prime}=1}{c} o_{a_{s^{\prime}}}
\end{array}\right\}
$$

Assim, baseando-se nas expressões de $\epsilon_{S}$ e de $E_{a_{s^{\prime}}}$, os elementos da matriz $\mathrm{M}^{-1}$ podem ser determinados. Ve rifica-se, então, que se $m_{s s} e m_{a^{\prime} s^{\prime}}$ são os elementos da diagonal principal de $\mathrm{M}^{-1}$, correspondentes aos tratamentos originais e adicionais, respectivamente, segue-se que:

$$
m_{s s}=\frac{k^{\prime}}{r\left(k^{\prime}-1\right)}, \quad m_{a_{s^{\prime}} s^{\prime}}=\frac{1}{b}
$$

Por outro lado, os elementos de $\mathrm{M}^{-1}$, fora da diagonal, são: 


$$
\begin{aligned}
& m_{S^{*}}=m_{s^{*}}= \frac{k^{\prime}}{r\left(k^{\prime}-1\right)} \sum_{i=1}^{m} \lambda_{i} f_{i l} \text {, oara quaisquer dois tratamentos } \\
& \text { originais que são l-ésimos associados; } \\
& m_{s^{\prime}}=\frac{1}{r\left(k^{\prime}-1\right)}\left(\frac{r}{b}+\sum_{\ell=1}^{m}\left(\frac{r^{2}-\lambda_{l} b}{b r}\right) n_{\ell} \sum_{i=1}^{m} \lambda_{i} f_{i l}\right) \text { e } m_{s^{\prime} s}=0 \text {, para } \\
& \\
& \text { o s-ésimo tratamento original e o s'-ésimo tratamento } \\
& \text { adicional; }
\end{aligned}
$$

$m_{a_{s^{\prime} \mathbf{s}^{\prime *}}}=m_{a_{s^{\prime}: *_{s^{\prime}}}}=0$, para quaisquer dois tratamentos adicionais.

$$
\text { 3.6.1 - Esperança matemática de } \tilde{\tau}
$$

Considerando-se o modelo [3.2.a] sem o efeito da média, e usando-se o mesmo procedimento de PIMENTEL GOMES (1967), tem-se que:

$$
E(\underset{\sim}{\tau})=E\left(M^{-1} \underset{\sim}{Q}\right),
$$

onde $\underset{\sim}{Q}=W^{\prime} \underline{\sim}$, conforme relatado em $(3.5) ;$ logo,

$$
\begin{aligned}
E(\underset{\sim}{\tilde{\tau}}) & =M^{-1} W X^{\prime} E(\underset{\sim}{y}) \\
& =M^{-1} W X^{\prime} X \underset{\sim}{\theta}
\end{aligned}
$$


Mas,

$$
\begin{aligned}
W X^{\prime} X & =\left[I_{V^{\prime}}-N K^{-1}\right]\left(\begin{array}{cc}
R & N \\
N^{\prime} & K
\end{array}\right) \\
& =\left[R-N K^{-1} N^{\prime}, \phi\right] \\
& =[C, \phi]
\end{aligned}
$$

Voltando em [3.6.1.a], tem-se então que:

$$
\begin{aligned}
E(\underline{\tau}) & =M^{-1}\left[\begin{array}{ll}
C & \phi
\end{array}\right]\left(\begin{array}{l}
\tau \\
\tilde{\beta}
\end{array}\right) \\
& =M^{-1} \underline{\sim}_{\tilde{\tau}} \\
& =M^{-1}(M+A) \underline{\sim} \\
& =\underline{\tau}+M^{-1} A \underline{\tau}
\end{aligned}
$$

Supondo-se que a equação [3.5.1.b] para $\hat{\tau}$ é também satisfeita para $\tau_{\text {, então } A \tau}^{A \tau}=\phi$. Logo,

$$
E(\underline{\tau})=\underline{\tau},
$$

ou seja, $\underset{\tau}{ }$ é um estimador não tendencioso de $\tilde{\sim}$.

OBSERVAÇÃO: Em todas as expressões algébricas onde ocorrer a matriz w, considerar-se-á o modelo [3.2.a] sem o efeito da média, ou seja, a matriz $x$ será $x=\left[x_{2} \vdots x_{3}\right]$. 


\section{6 .2 - Matriz de dispersão para $\underline{\text { I }}$}

A matriz de dispersão do vetor $\tilde{\tau}$ é, por defi nição:

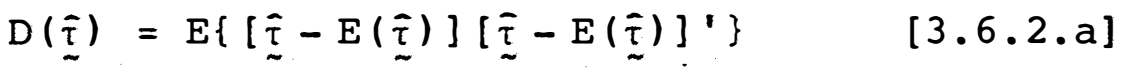

$$
\begin{aligned}
& \text { Como } \underset{\sim}{\bar{\tau}}=\mathrm{M}^{-1} \underline{\underline{Q}} \text { e } \underset{\sim}{Q}=\mathrm{WX}^{\prime} \underset{\sim}{\mathrm{y}} \text {, tem-se então que: } \\
& \bar{\tau}=M^{-1} W X^{\prime} \underline{y} \\
& =M^{-1} W^{\prime}(X \underset{\sim}{\theta}+\underset{\sim}{\varepsilon}) \\
& =M^{-1} W X^{\prime} X_{\underset{\theta}{\theta}}+M^{-1} W^{\prime} \underset{\sim}{\varepsilon} \cdot \quad[3 \cdot 6.2 . b]
\end{aligned}
$$

Portanto, considerando-se [3.6.1.a] e [3.6.2.b],

obtém-se:

$$
\bar{\tau}-E(\tilde{\tau})=M^{-1} W X^{\prime} \varepsilon
$$

Substituindo-se $[3,6.2 . c]$ em $[3.6 .2 . a]$, segue-se que:

$$
D(\tilde{\tau})=E(M^{-1} W X^{\prime} \underset{\sim}{\varepsilon} \underbrace{\prime} X W^{\prime} M \bar{r}^{-1})
$$

Como admite-se em (3.2) que $E\left(\underline{\varepsilon}^{\prime}\right)=\sigma^{2} I$, en tão,

$$
D(\underset{\sim}{\bar{\tau}})=M^{-1} W X^{\prime} X W^{\prime} M^{-1} \sigma^{2},
$$$$
[3 \cdot 6.2 . d]
$$ 
que, por [3.6.1.b], fica:

$$
D(\underline{\tau})=M^{-1} \mathrm{CM}^{-1} \sigma^{2} \ldots \quad[3.6 .2 . e]
$$

Se $\underline{\sim}^{\prime}$ ̃ è uma função linear, então a variância de $\underset{\sim}{p}{ }^{\prime} \tilde{\sim}$ é dada por:

$$
\begin{aligned}
& \operatorname{Var}\left(\underline{\sim}^{\prime} \hat{\tau}\right)=E\left[\left\{\underline{\sim}^{\prime} \bar{\tau}^{\bar{\tau}}-E\left(\underline{\sim}^{\prime} \bar{\tau}\right)\right]\left[\underline{\sim}^{\prime} \bar{\tau}-E\left(\underline{\sim}^{\prime} \underline{\tau}\right)\right]^{\prime}\right\} \quad[3.6 .2 . f] \\
& =\underline{\sim}^{\prime} E\left\{[\underline{\tilde{\tau}}-E(\underline{\tilde{\tau}})][\underline{\tilde{\tau}}-E(\underline{\underline{\tau}})]^{\prime}\right\} \underset{\sim}{p} \\
& =\underset{\sim}{p} D(\underline{\tau}) \underset{\sim}{p} \\
& ={\underset{\sim}{p}}^{\prime} M^{-1} C M^{\top 1} \underset{\sim}{p} \sigma^{2}
\end{aligned}
$$

Se $\underset{\sim}{p}$ ' é uma função linear estimāvel, então $\underline{\sim}^{\prime}=\underline{\sim}^{\prime} C$, ou seja, $\underset{\sim}{p}=C_{\sim}$, onde $\underset{\sim}{a}$ é um vetor. Assim, confor me PIMENTEL GOMES (1967), a expressão [3.6.2.g] fica:

$$
\begin{aligned}
& \operatorname{Var}\left(\underset{\sim}{p}{ }_{\sim}^{\top}\right)=\underset{\sim}{p} M^{-1} \mathrm{CM}^{-1} \mathrm{Ca}_{\sim} \sigma^{2} \quad \cdot \quad[3 \cdot 6 \cdot 2 . \mathrm{h}] \\
& \text { Por outro lado, OGAWA (1974) e IEMMA (1985) }
\end{aligned}
$$

mostram que a matriz $\mathrm{M}^{7^{1}}$ é uma inversa condicional de C. Lo go,

$$
C M^{-1} \mathrm{C}=\mathrm{C}
$$

Sendo C uma matriz simétrica, segue-se que:

$$
\mathrm{CM}^{-1} \mathrm{C}=\mathrm{C}
$$


Substituindo-se $[3.6 .2 . j]$ em $[3.6 .2 . h]$, ob-

tém-se:

$$
\operatorname{Var}(\underset{\sim}{p} \cdot \tilde{q})=\underline{\sim}^{\prime} M^{-1} \operatorname{Ca}_{\sim} \sigma^{2}
$$

Mas, $\mathrm{Ca}=\underset{\sim}{\mathrm{p}} ; \log 0$,

$$
\operatorname{Var}\left(\underset{\sim}{p} \tilde{\tau}^{\prime}\right)=\underline{\sim}^{\prime} M^{-1} \underline{p} \sigma^{2} . \quad[3.6 .2 . \mathrm{k}]
$$

E conveniente mostrar tambēm que:

$$
\begin{aligned}
A M^{-1} W X^{\prime} & =(C-M) M^{-1} W X^{\prime} \\
& =C M^{-1} W X^{\prime}-W X^{\prime}
\end{aligned}
$$

Pós-multiplicando-se a expressão anterior por $\mathrm{XW}$ ' $M{ }^{-1} \mathrm{~A}$ ', tem-se:

$$
\begin{gathered}
A M^{-1} W X^{\prime} X W^{\prime} M^{-1} A^{\prime}=C M^{-1} W X^{\prime} X W^{\prime} M^{-1} A^{\prime}-W X^{\prime} X W^{\prime} M^{-1} A^{\prime}, \\
\text { Por }[3.6 .1 . b] \text { chega-se a } W X^{\prime} X W^{\prime}=C ; 10 g o, \\
A M^{-1} W X^{\prime} X W^{\prime} M^{-1} A^{\prime}=C M^{-1} C M^{-1} A^{\prime}-C M^{-1} A^{\prime},
\end{gathered}
$$

que, por [3.6.2.i], resulta em:

$$
A M^{-1} W X^{\prime} X W^{\prime} M^{-1} A^{\prime}=\phi,
$$

ou seja,

$$
A M^{-1} W X^{\prime}=\phi .
$$


3.7 - Somas de quádrados

$$
\begin{aligned}
& \text { Do modelo } \underset{\sim}{\mathrm{y}}=\underset{\sim}{\mathrm{x}}+\underset{\sim}{\varepsilon} \text {, obtém-se } \\
& \underline{\sim}^{\prime} \underline{\sim}=\underline{y^{\prime}} \underline{\sim}-\underline{\sim}^{\prime} X^{\prime} \underline{y},
\end{aligned}
$$

e tomando-se a estimativa de $\theta$, resulta a soma de quadrados do residuo, ou seja:

$$
S Q R=\underline{x}^{\prime} \underset{\sim}{y}-\underline{\sim}^{\prime} X^{\prime} \underline{\sim},
$$

onde, $\underline{\sim}^{\prime} \mathrm{x} \underline{\sim}^{\prime}$, definida como a soma de quadrados de parâmetros, é:

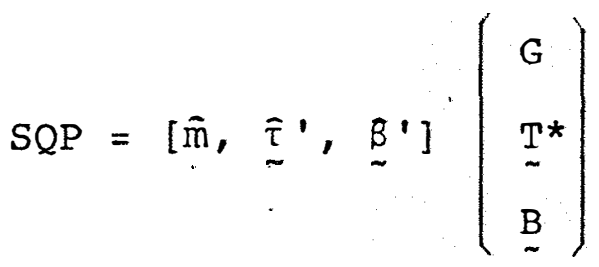

$$
\begin{aligned}
& =\hat{\mathrm{m}} G+\tilde{\tau}^{\prime} \underline{\sim}^{*}+\underset{\sim}{B}{ }_{\sim}^{B} .
\end{aligned}
$$

De $[3.4 . e]$ tem-se que $\underset{\sim}{B}=K^{-1}\left(\underset{\sim}{\left(\underset{B}{z} \hat{m}-N^{\prime}\right.} \underset{\sim}{\tau}\right) ; 10-$ go,

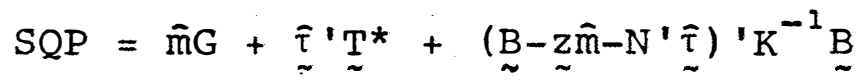

$$
\begin{aligned}
& =\hat{\mathrm{m}} G+\stackrel{\sim}{\hat{\tau}}^{T^{*}}{ }^{*}+\underset{\sim}{B^{\prime}} K^{-1} \underset{\sim}{B}-\hat{m} \underset{\sim}{z} K^{-1} \underset{\sim}{B}-\underset{\sim}{\hat{\tau}}{ }^{\prime} N K^{-1} \underset{\sim}{B} \\
& =\hat{\mathrm{m}} \mathrm{G}+\hat{\tau}^{\prime}\left(\mathrm{T}^{*}-\frac{1}{\mathrm{k}^{\prime}} \underset{\sim}{\mathrm{NB}}\right)+\frac{1}{\mathrm{k}^{\prime}} \stackrel{\sim}{\mathrm{B}}^{\prime} \stackrel{\sim}{\mathrm{B}}-\hat{\mathrm{m} G} \\
& =\hat{\mathrm{m}} G+\hat{\tau}^{\prime} \underline{\sim}+\frac{1}{k^{\prime}}{\underset{\sim}{B}}^{\prime} \underline{\sim}^{\prime}-\hat{m} G
\end{aligned}
$$


Substituindo-se [3.7.b] em [3.7.a], tem-se:

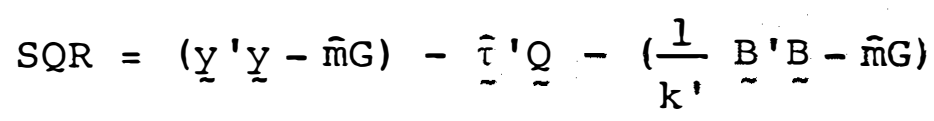

onde,

$\underline{\sim}^{\prime} \underline{y}-\hat{m} G$ é a soma de quadrados total (SQT);

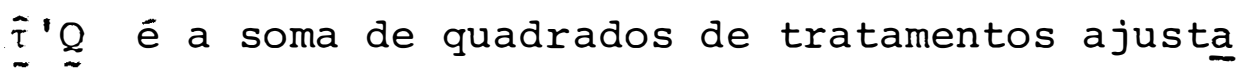
da para blocos (SQTaj.);

$\underline{B}^{\prime} K^{-1} \underset{\sim}{B}-\bar{m} G$ é a soma de quadrados de blocos ( $S \Omega B$ ).

$$
\text { Sabe-se que } \tilde{\tau}^{\prime}=\left[\underline{\underline{\tau}}_{1}^{\prime}, \hat{\tau}_{2}^{\prime}\right] \text { e } \underline{Q}^{\prime}=\left[\underline{\sim}_{1}^{\prime}, \quad \underline{\sim}_{2}^{\prime}\right] \text {. }
$$

Logo,

$$
\tilde{\tau}^{\prime} \underline{\sim}=\tilde{\sim}_{1}^{\prime} \stackrel{\sim}{1}_{1}+\bar{\tau}_{2}^{\prime} \stackrel{\sim}{2}_{2},
$$

ou seja,

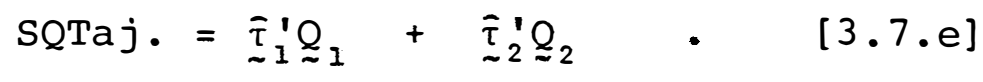

Considerando-se a forma usual de apresentação das sonas de quadrados, tem-se que:

$$
\begin{aligned}
\text { SQT } & =\sum_{u, h} y_{u h}^{2}-C_{0}, \\
\text { SQTaj. } & =\sum_{s=1}^{v} E_{s^{Q} s}+\sum_{s^{\prime}=1}^{c} E_{a_{S^{\prime}}} Q_{a_{S^{\prime}},}, \\
\text { SQB } & =\frac{1}{k^{\prime}} \sum_{h=1}^{b} B_{h}^{2}-C_{0}, \\
\text { SQR } & =\text { SQT }- \text { SQTaj. }- \text { SQB, }
\end{aligned}
$$

onde $C_{0}=\hat{m} G$ é a soma de quadrados da média geral (correção) . 
Tomando-se $[3.5 .2 .1 . e],[3.5 .2 .2 . v]$ e $[3.5 .2 .3 . h]$, a SQTaj. pode ser apresentada na seguinte forma:

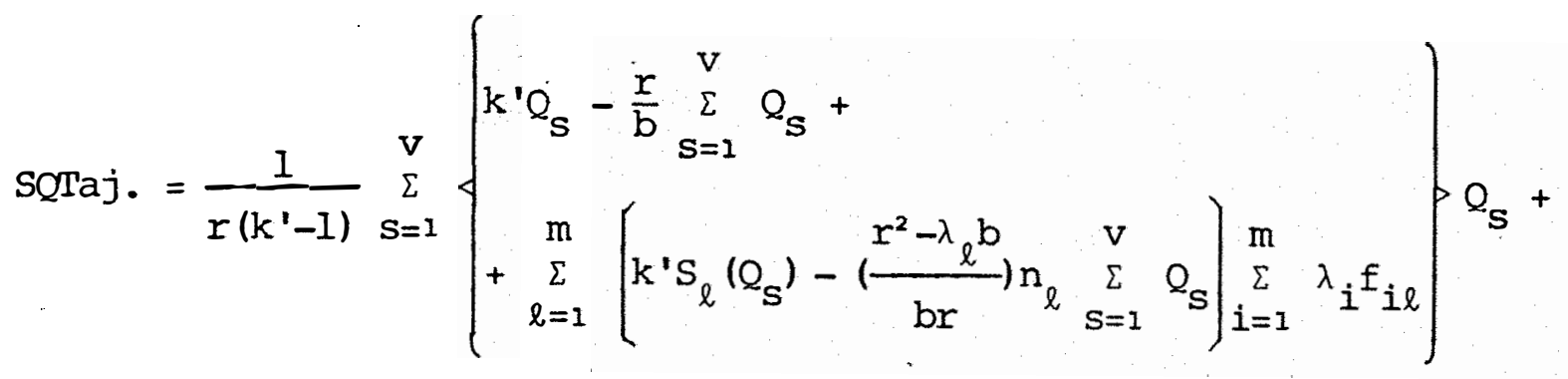

$$
\begin{aligned}
& +\frac{1}{b}\left(\sum_{s^{\prime}=1}^{c} Q_{a_{s^{\prime}}}\right) Q_{a_{s^{\prime}}}
\end{aligned}
$$

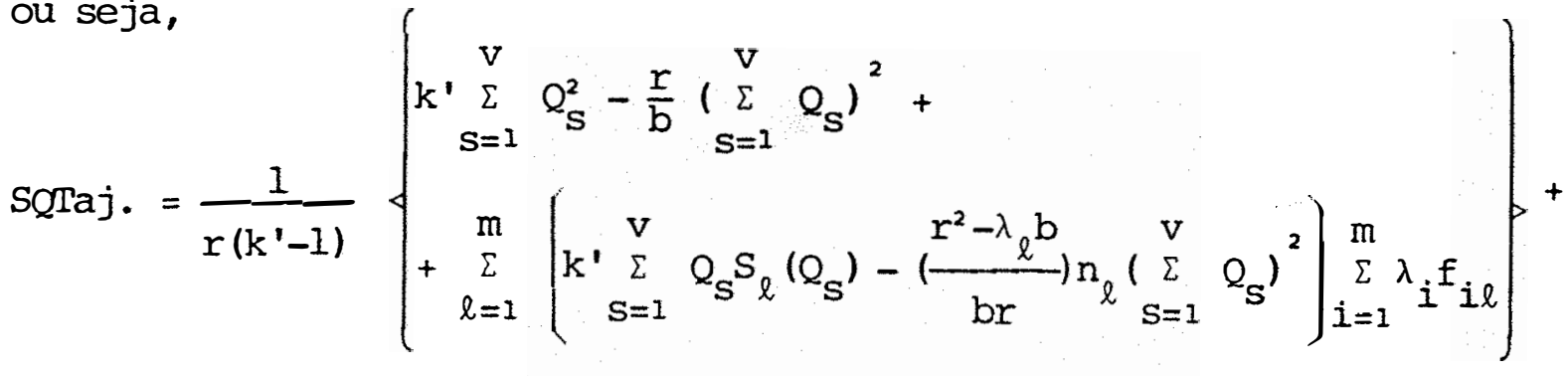

$$
\begin{aligned}
& +\frac{1}{b} \sum_{S^{\prime}=1}^{c} Q_{S_{S^{\prime}}}
\end{aligned}
$$

Por outro lado, sabe-se que a soma de quadra dos dos tratamentos adicionais (SQTad.) ê dada de modo usual, por:

$$
\text { SQTad. }=\frac{1}{b} \sum_{s^{\prime}=1}^{c} T_{a_{s^{\prime}}}^{2}-\frac{1}{b c}\left(\sum_{s^{\prime}=1}^{c} T_{a_{s^{\prime}}}\right)^{2} \text {. }
$$$$
\text { Sabe-se ainda que } Q_{a_{s^{\prime}}}=T_{a_{s^{\prime}}}-\frac{G}{k^{\prime}} ; \text { logo, }
$$ 


$$
\text { sqTad. }=\frac{1}{b} \underset{s^{\prime}=1}{c}\left(Q_{a_{s^{\prime}}}+\frac{G}{k^{\prime}}\right)^{2}-\frac{1}{b c}\left(\sum_{s^{\prime}=1}^{c} Q_{a_{s^{\prime}}}+\frac{c G}{k^{\prime}}\right)^{2} .
$$

que resulta em:

$$
\text { sQTad. }=\frac{1}{b} \underset{S^{\prime}=1}{c} Q_{a_{S^{\prime}}}^{2}-\frac{1}{b c}\left(\underset{s^{\prime}=1}{c} Q_{a_{S^{\prime}}}\right)^{2}
$$

Considerando-se [3.7.i] em [3.7.h], tem-se então:

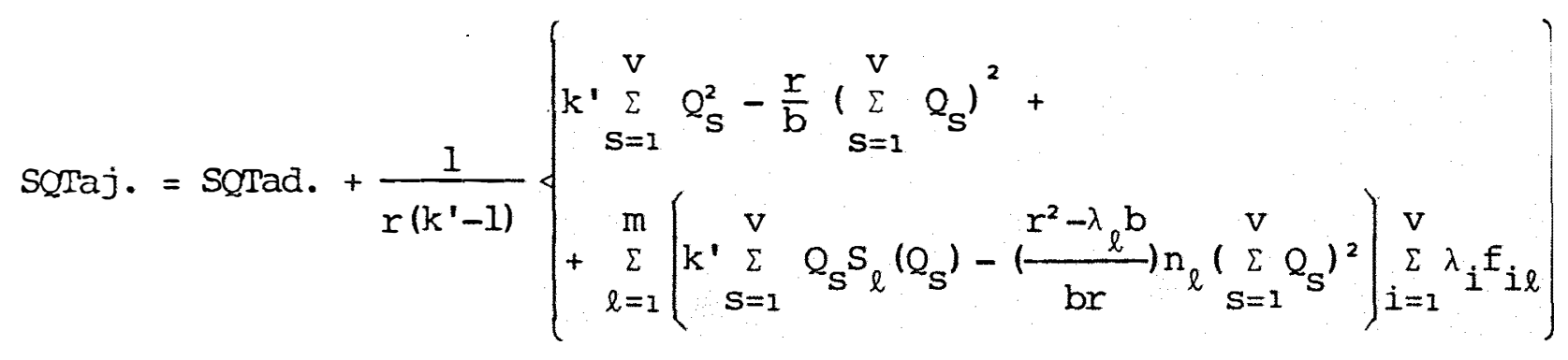

$$
+\frac{1}{b c}\left(\sum_{s^{\prime}=1}^{c} Q_{a_{s^{\prime}}}\right)^{2}
$$

$$
=\text { SQTad. }+\frac{1}{r\left(k^{\prime}-1\right)}\left\{\begin{array}{l}
k^{\prime} \sum_{S=1}^{v} Q_{S}^{2}+\frac{1}{b c}\left[r\left(k^{\prime}-1\right)-r c\right]\left(\sum_{S=1}^{v} Q_{S}\right)^{2}+ \\
\sum_{l=1}^{m}\left(k^{\prime} \sum_{S=1}^{v} Q_{S} S_{\ell}\left(Q_{S}\right)-\left(\frac{r^{2}-\lambda \ell^{b}}{b r}\right) n_{\ell}\left(\sum_{S=1}^{v} Q_{S}\right)^{2}\right) \sum_{i=1}^{m} \lambda_{i} f_{i \ell}
\end{array}\right.
$$

A soma de quadrados entre os dois tipos de tratamentos (originais e adicionais) é dada de modo usual, por: 


$$
\text { SQTipos }=\frac{1}{b k}\left(\sum_{s=1}^{v} T_{s^{\prime}}\right)^{2}+\frac{1}{b c}\left(\underset{s^{\prime}=1}{c} T_{a_{s^{\prime}}}^{c}\right)^{2}-\frac{G^{2}}{b k^{\prime}}
$$

Mas,

$$
\sum_{S=1}^{v} T_{S}=\sum_{S^{\prime}=1}^{v} Q_{S}+\frac{k G}{k^{\prime}} \text { e } \sum_{S^{\prime}=1}^{c} T_{a_{S^{\prime}}}=\sum_{S^{\prime}=1}^{c} Q_{a_{S^{\prime}}}+\frac{\mathrm{cG}}{k^{\prime}} .
$$

Logo,

$$
\text { SQTipos }=\frac{1}{b k}\left(\sum_{S^{\prime}=1}^{v} Q_{S}+\frac{k G}{k^{\prime}}\right)^{2}+\frac{I}{b c}\left(\sum_{s^{\prime}=1}^{c} Q_{a_{S^{\prime}}}+\frac{c G}{k^{\prime}}\right)-\frac{G^{2}}{b k^{\prime}},
$$

que resulta em

$$
\begin{aligned}
& \text { SQTipos }=\frac{1}{b k}\left(\sum_{S=1}^{v} Q_{s}\right)^{2}+\frac{1}{b c}\left(\sum_{S^{\prime}=1}^{c} Q_{a_{S^{\prime}}}\right)^{2}
\end{aligned}
$$

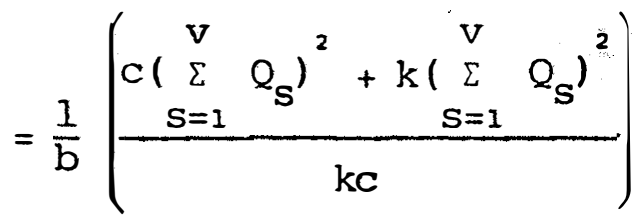

Como $c+k=k^{\prime}$, segue-se que:

$$
\begin{aligned}
\text { SQTipos } & =\frac{k^{\prime}}{b k c}\left(\underset{s=1}{\sum_{S}} Q_{S}\right)^{2} \\
& =\frac{k^{\prime}}{r v c}\left(\underset{S=1}{v} Q_{S}\right)^{2}
\end{aligned}
$$


SQTaj. = SQTad. + SQTipos +

$$
+\frac{1}{r\left(k^{\prime}-1\right)}\left\{\begin{array}{l}
k^{\prime} \sum_{S=1}^{v} Q_{S}^{2}-\left(\frac{r}{b}+\frac{\left(k^{\prime}-1\right)}{v}\right)\left(\sum_{S=1}^{v} Q_{S}\right)^{2}+ \\
\left.+\sum_{l=1}^{m}\left(k^{\prime} \sum_{S=1}^{v} Q_{S} S_{\ell}\left(Q_{S}\right)-\left(\frac{r^{2}-\lambda l_{l}^{b}}{b r}\right) n_{\ell}\left(\sum_{S=1}^{v} Q_{S}\right)^{2}\right) \sum_{i=1}^{m} \lambda_{i} f_{i \ell}\right)
\end{array}\right\}
$$

ou ainda,

SQTaj. = SQTad. + SQTipos +

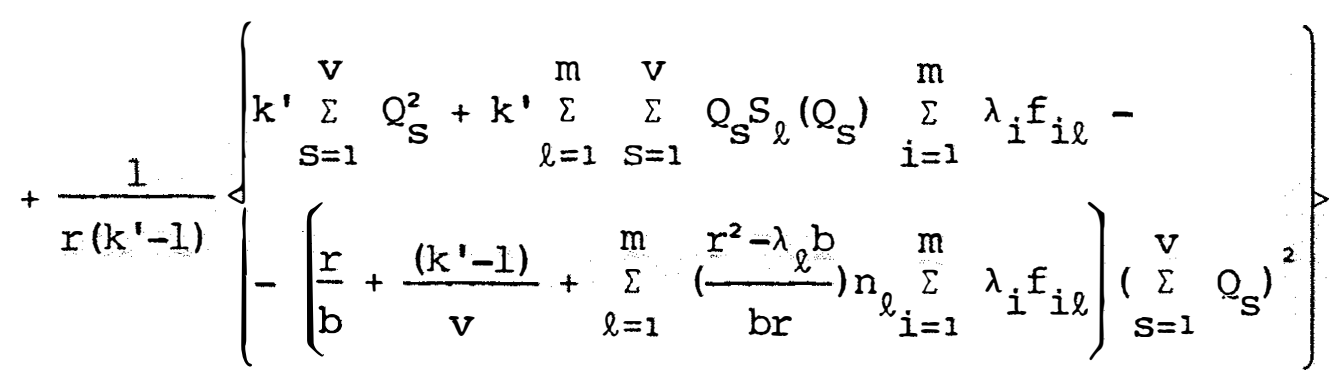

sendo que a última expressão corresponde à soma de quadrados dos tratamentos originais ajustadas, sQTor. (aj.), visto que:

$$
\text { SQTaj. = SQTad. + SQTipos + SQTor. (aj.) }
$$

Como mencionado em [3.5.2.3], os delineamentos PBIB com duas classes de associados, $\mathrm{m}=2$, são de parti- 
cular interesse. Nesse caso, a soma de quadrados dos tratamentos originais ajustada, fica:

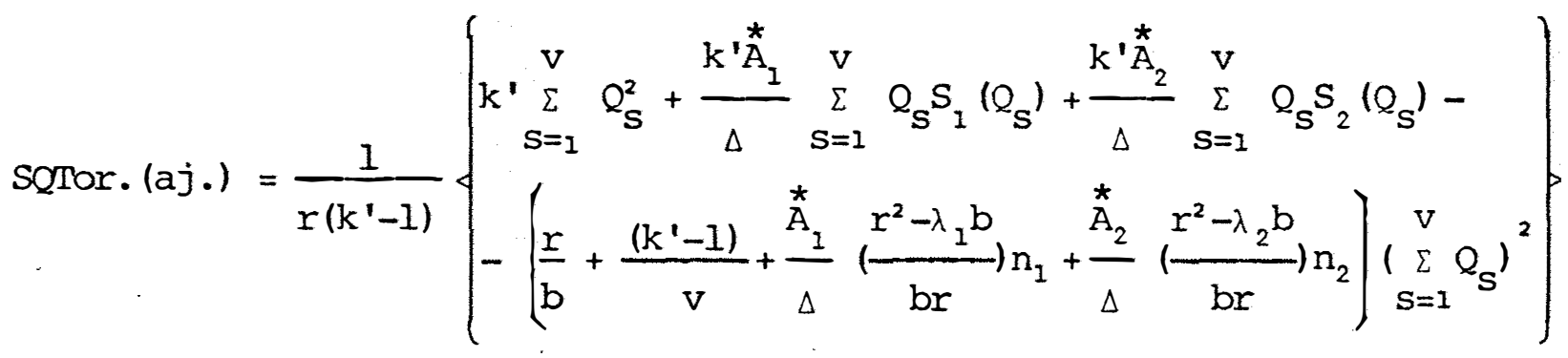

Sabe-se que $\sum_{S=1}^{v} Q_{S}=Q_{S}+S_{1}\left(Q_{S}\right)+S_{2}\left(Q_{s}\right)$. Assim, se $n_{1}<n_{2}$, é conveniente substituir $S_{2}\left(Q_{S}\right)$, em [3.7.0] por $\mathrm{v}$ $\sum_{S=1} Q_{S}-Q_{S}-S_{1}\left(Q_{S}\right)$. Isso resulta em:

$$
\text { s=1 }
$$

SQTor. (aj.) $=\frac{1}{r\left(k^{\prime}-1\right) \Delta}$

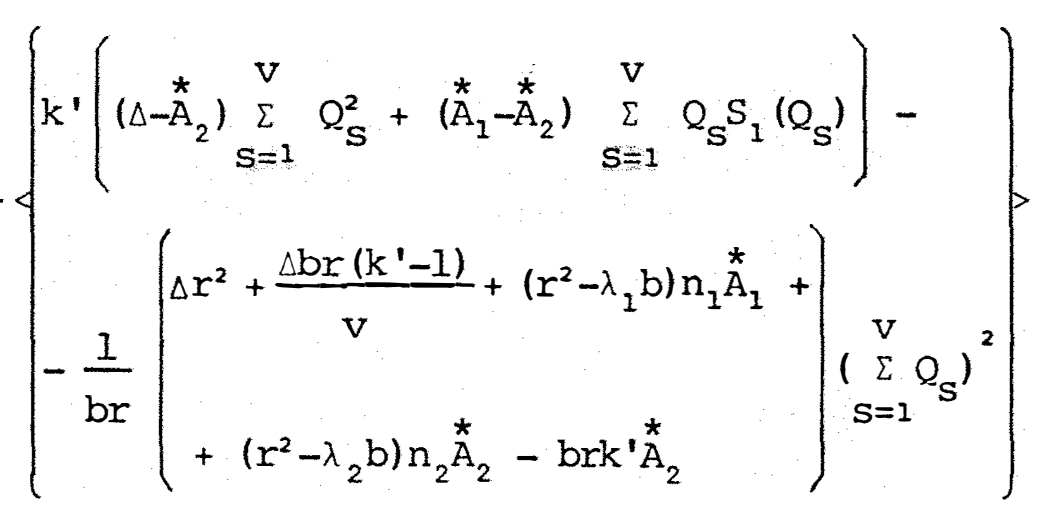$$
\text { , }
$$

Para o caso onde $\mathrm{n}_{2}<\mathrm{n}_{1}$, deve-se substituir $S_{1}\left(Q_{S}\right)$, em [3.7.0], por $\sum_{s=1}^{V} Q_{S}-Q_{S}-S_{2}\left(Q_{S}\right)$. Isso resulta em: 


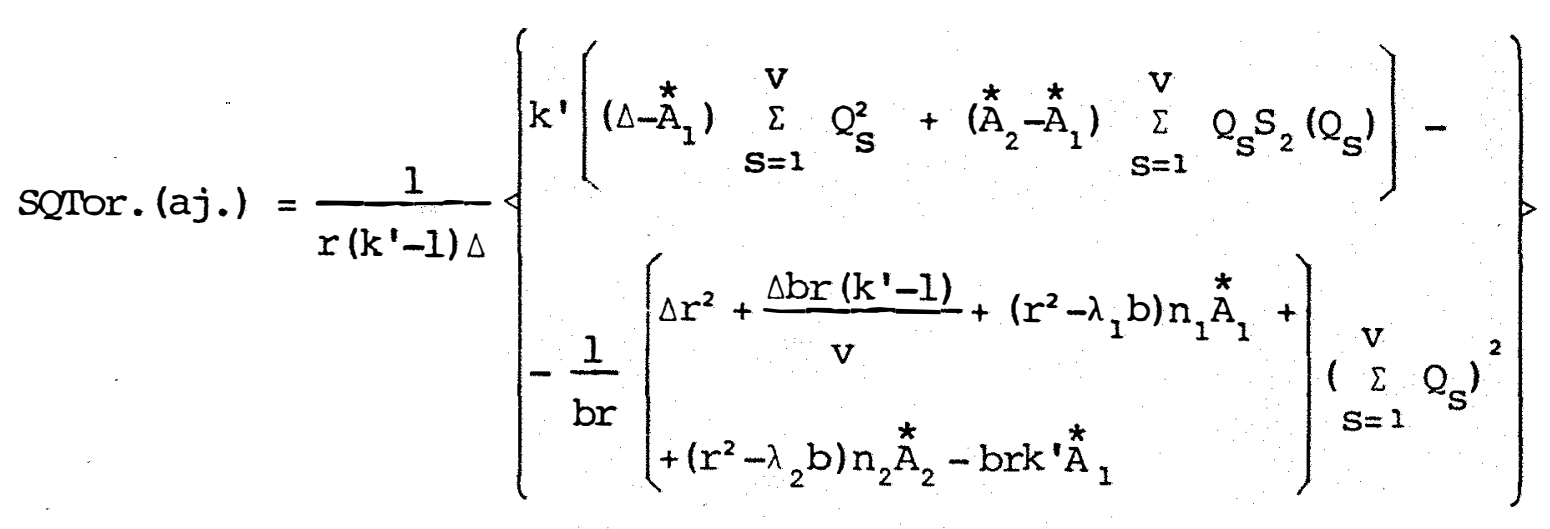

$[3.7 . q]$

Quando os tratamentos originais estão dispos tos em delineamento BIB, a expressão para a SQTor.(aj.) sim plifica-se, visto que, nesse caso, $\lambda_{1}=\lambda_{2}=\ldots=\lambda_{m}$. Tomando-se como base o PBIB, com duas classes de associados, conforme em [3.5.2.5], os parāmetros modificam-se para:

$$
\begin{array}{ll}
a_{11}=a_{22}=r\left(k^{\prime}-1\right)+\lambda, & a_{12}=a_{21}=0, \\
\Delta=\left[r\left(k^{\prime}-1\right)+\lambda\right]^{2}, & \stackrel{*}{A}{ }_{1}=\stackrel{\star A}{A}_{2}=\left[r\left(k^{\prime}-1\right)+\lambda\right] \lambda, \\
n_{1}+n_{2}=v-1, &
\end{array}
$$

e a soma de quadrados dos tratamentos originais ajustada, passa a ser:

$$
\text { SQTOr. }(a j \cdot)_{B I B}=\frac{k^{\prime}}{\lambda v+r C}\left(\sum_{s=1}^{v} Q_{S}^{2}-\frac{I}{v}\left(\sum_{s=1}^{v} Q_{s}\right)^{2}\right) \quad[3 \cdot 7 \cdot r]
$$

que ẽ a expressão obtida por KALIN (1966) e FERREIRA (1980). 
3.8 - Esperanças Matemáticas das Somas de Quadrados

3.8.1 - Esperança matemática da sôm

Sabe-se que a soma de quadrados da média geral (SQm) é dada por:

$$
\begin{aligned}
\text { SQ⿳亠丷⿵冂丶 } & =\hat{\mathrm{m}} \mathrm{G} \\
& =\frac{G^{2}}{\mathrm{n}} .
\end{aligned}
$$

Logo, tem-se:

$$
\begin{aligned}
& E(S Q \hat{m})=\frac{1}{n} E\left[\left(X_{1}^{\prime} \underset{\sim}{y}\right) \cdot\left(X_{1}^{\prime} \underset{\sim}{y}\right)\right] \\
& =\frac{1}{n} E\left[({\underset{\sim}{\theta}}^{\prime} X^{\prime}+\underbrace{\prime}_{\sim}) X_{1} X_{1}^{\prime}(\underset{\sim}{x}+\underset{\sim}{\varepsilon})\right] \\
& =\frac{1}{n} E\left[\underset{\sim}{\theta^{\prime}} X^{\prime} X_{1} X_{1}^{\prime} X \underset{\sim}{\theta}+\underset{\sim}{\theta} X^{\prime} X_{1} X_{1}^{\prime} \underset{\sim}{\varepsilon}+{\underset{\sim}{\varepsilon}}^{\prime} X_{1} X_{1}^{\prime} x_{\sim}^{\theta}+{\underset{\sim}{\varepsilon}}^{\prime} X_{1} X_{1}^{\prime} \underset{\sim}{\varepsilon}\right] \\
& \left.=\frac{1}{n} \underset{\sim}{\theta^{\prime}} X^{\prime} x_{1} x_{1}^{\prime} x_{\sim}^{\theta}+E\left(\underline{\sim}^{\prime} X_{1} X_{1}^{\prime} \underset{\sim}{\varepsilon}\right)\right],
\end{aligned}
$$

onde,

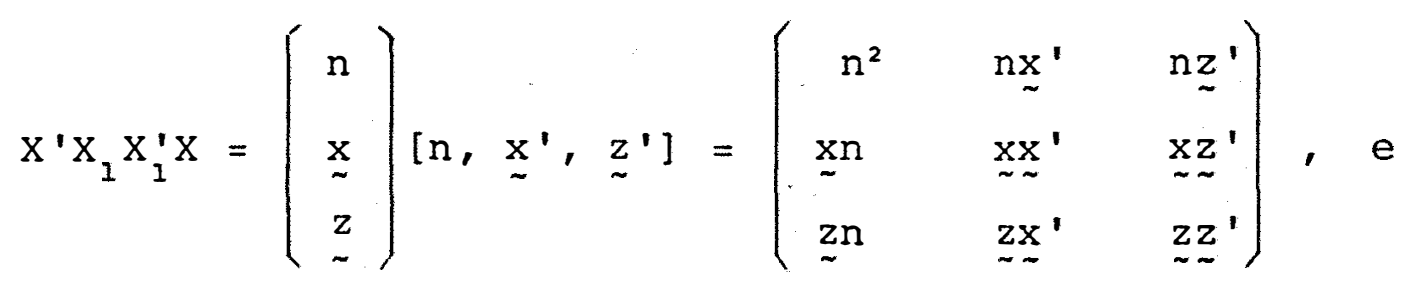




$$
\begin{aligned}
& \underline{\sim}^{\prime} x^{\prime} x_{1} x_{1}^{\prime} x \underline{\sim}=\left(m n^{2}+\tau^{\prime} x n+\underline{\sim} \underline{\sim}^{\prime} z m\right) m+ \\
& +\left(m \underline{\sim}^{\prime}+\tau^{\prime} x x^{\prime}+\underline{\sim}^{\prime} z \underline{\sim}^{\prime}\right) \underline{\sim}+ \\
& +\left(m n z^{\prime}+\tau^{\prime} x z^{\prime}+\underline{\sim}^{\prime} z z_{\sim}^{\prime}\right) \underset{\sim}{\beta} \\
& =m^{2} n^{2}+\tau^{\prime} x n m+\beta^{\prime} z n m+m n x^{\prime} \tau+\tau^{\prime} x x^{\prime} \tau+ \\
& +\underline{\beta}^{\prime} z x^{\prime} \tau+m n z^{\prime} \underline{\sim}+\underline{\tau}^{\prime} \underline{x z}^{\prime} \underline{\sim}+\beta^{\prime} z z^{\prime} \underline{\sim}
\end{aligned}
$$

Retomando-se as restrições usuais, $\underset{\sim}{x^{\prime} \tau}=0$ e $\underline{z}^{\prime} \underline{\sim}=0$, segue-se que:

$$
\underset{\sim}{\theta} x^{\prime} x_{1} x_{1}^{\prime} x \underset{\sim}{\theta}=m^{2} n^{2}
$$

Assim, voltando em [3.8.1.a],

$$
\begin{aligned}
E(S Q \hat{m}) & =n m^{2}+\frac{1}{n} E\left(\underset{\sim}{\varepsilon} x_{1} x_{1}^{\prime} \underset{\sim}{\varepsilon}\right) \\
& =n m^{2}+\frac{1}{n} E\left[\operatorname{TR}\left(X_{1} X_{1}^{\prime} \underset{\sim}{\varepsilon} \varepsilon^{\prime}\right)\right] \\
& =n m^{2}+\frac{1}{n} \operatorname{TR}\left[X_{1} X_{1}^{\prime} E\left(\underset{\sim}{\varepsilon} \varepsilon^{\prime}\right)\right]
\end{aligned}
$$

Mas, $E\left(\underset{\sim}{\varepsilon E^{\prime}}\right)=\sigma^{2} I$ e $\operatorname{TR}\left(X_{1} X_{1}^{\prime}\right)=n$, então,

$$
E(S Q \hat{m})=E\left(C_{0}\right)=n m^{2}+\sigma^{2} \quad \cdot \quad[3.8 .1 . C]
$$




\section{8 .2 - Esperança matemática de $\tilde{\tau}^{\prime} Q$}

Sabe-se que:

$$
\underline{\hat{\tau}}^{\prime} \underline{Q}=\underline{Q}^{\prime} M T^{-1} \underline{Q} .
$$

Mas, de (3.5), $\underset{\sim}{Q}=W^{\prime} \underset{\sim}{y}$, então, considerando-se o modelo [3.2.a] sem o efeito da média, como PIMENTEL GO MES (1967), segue-se que:

$$
\begin{aligned}
& E\left(\underset{\sim}{(\tilde{\tau}}{ }^{Q}\right)=E\left[\underline{Z}^{\prime} X W^{\prime} M^{-1} W X^{\prime} \underline{\underline{y}}\right) \\
& =E\left[({\underset{\sim}{\theta}}^{\prime} X^{\prime}+\underbrace{\prime}_{\sim}) X W^{\prime} M^{-1} W X^{\prime}(X \underset{\sim}{\theta}+\underset{\sim}{\varepsilon})\right] \\
& =\underline{\theta}^{\prime} X^{\prime} X W^{\prime} M{ }^{-1} W X^{\prime} X \underset{\sim}{\theta}+E(\underbrace{\prime} X W^{\prime} M^{T^{-1}} W X^{\prime} \underline{\sim}) \\
& \text { Por }[3.6 .1 . b] \text { e }[3.6 .2 . j] \text {, obtém-se: } \\
& E(\underset{\sim}{\tilde{\tau}} \underset{\sim}{Q})={\underset{\sim}{ }}^{\prime} C_{\sim}^{\tau}+E\left(\underset{\sim}{\varepsilon} X W^{\prime} M^{-1} W^{\prime} X^{\prime} \underset{\sim}{ }\right) \\
& =\underset{\sim}{\tau}{ }^{\prime} \underset{\sim}{\tau}+E\left[T R\left(X W W^{\prime} T^{-1} W X^{\prime} \underset{\sim}{\varepsilon} \tilde{\sim}^{\prime}\right)\right] \\
& =\sim^{\prime} C_{\sim}^{\prime}+\operatorname{TR}\left[X W^{\prime} M T^{-1} W X^{\prime} E\left(\underset{\sim}{\varepsilon} \varepsilon^{\prime}\right)\right] \\
& =\underset{\sim}{\tau^{\prime}} \underset{\sim}{\tau}+\operatorname{TR}\left(M^{\top}{ }^{1} W X X^{\prime} X W^{\prime}\right) \sigma^{2} \\
& =\tilde{\tau}^{\prime}{ }_{\sim} \tau+\operatorname{TR}\left(M^{\top}{ }^{-1} C\right) \sigma^{2}
\end{aligned}
$$


Como $M^{-1} C$ é uma matriz idempotente, tem-se:

$$
\begin{aligned}
\operatorname{TR}\left(M^{T^{-1}} C\right) & =\text { caract. }\left(M^{T^{-1}} C\right) \\
& =\text { caract. }(C) \\
& =V^{\prime}-1
\end{aligned}
$$

Logo,

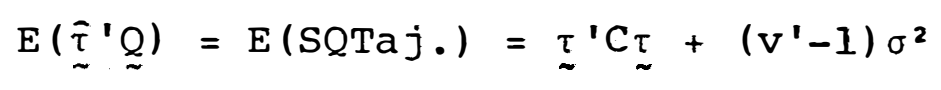

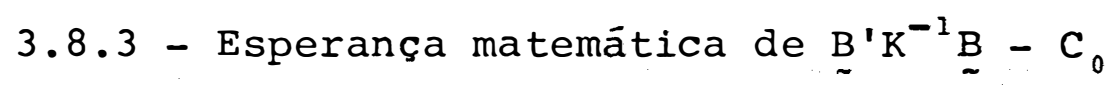

A soma de quadrados de blocos é dada por:

$$
\begin{aligned}
\mathrm{SQB} & =\underline{\sim}^{\prime} \mathrm{K}^{-1} \stackrel{\mathrm{B}}{\sim}-\mathrm{C}_{0} \\
& =\underline{\sim}^{\prime} \mathrm{X}_{3} \mathrm{~K}^{-1} \mathrm{X}_{3}^{\prime} \underset{\sim}{\mathrm{y}}-\mathrm{C}_{0}
\end{aligned}
$$

Tem-se, então, que:

$$
\begin{aligned}
& E(S Q B)=E\left(\underline{\sim}^{\prime} X_{3} K^{-1} x_{3}^{\prime} \underset{\sim}{y}\right)-E\left(C_{0}\right) \\
& =E\left[\left(\underline{\sim}^{\prime} X^{\prime}+\underline{\sim}^{\prime}\right) X_{3} K^{-1} X_{3}^{\prime}(X \underline{\theta}+\underline{\varepsilon})\right]-E\left(C_{0}\right) \\
& =\underset{\sim}{\theta} X^{\prime} X_{3} K^{-1} X_{3}^{\prime} X \underset{\sim}{\theta}+E\left(\underline{\varepsilon}^{\prime} X_{3} K^{-1} X_{3}^{\prime} \underset{\sim}{\varepsilon}\right)-E\left(C_{0}\right), \\
& {[3.8 \cdot 3 . a]}
\end{aligned}
$$


onde,

$$
\begin{aligned}
& x^{\prime} x_{3}=\left(\begin{array}{l}
x_{1}^{\prime} \\
x_{2}^{\prime} \\
x_{3}^{\prime}
\end{array}\right) \quad x_{3}=\left(\begin{array}{c}
z^{\prime} \\
N \\
K
\end{array}\right) \\
& X^{\prime} X_{3} K^{-1} X_{3}^{\prime} X=\left(\begin{array}{ccc}
\underset{\sim}{z} K^{-1} \underset{z}{z} & {\underset{\sim}{\prime}}^{\prime} K^{-1} N^{\prime} & \underset{\sim}{z} \\
N^{-1} \underset{z}{z} & N^{-1} N^{\prime} & N \\
\underset{\sim}{z} & N^{\prime} & K
\end{array}\right) \\
& \underset{\sim}{\theta} X^{\prime} X_{3} K^{-1} X_{3}^{\prime} X_{\sim}^{\theta}=m^{2}{\underset{\sim}{z}}^{\prime} K^{-1} \underset{\sim}{z}+\underset{\sim}{\tau} N^{-1} \underset{\sim}{z m}+\underset{\sim}{\beta}{ }_{\sim}^{\prime z m}+
\end{aligned}
$$

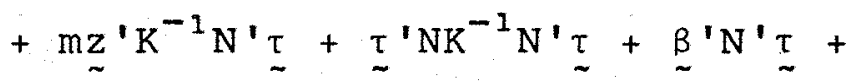

$$
\begin{aligned}
& +\mathrm{m}_{\sim}^{\prime}{ }_{\sim}^{\beta}+{\underset{\sim}{\tau}}^{\prime}{\underset{\sim}{\beta}}^{\beta}+\underset{\sim}{\beta}{ }^{\prime} \mathrm{K} \underset{\sim}{\beta}
\end{aligned}
$$

Impondo-se as restrições, conforme em [3.8.1]

tem-se:

$$
\begin{aligned}
& \underline{\sim}^{\prime} X^{\prime} X_{3} K^{-1} X_{3}^{\prime} X \underset{\sim}{\theta}=n m^{2}+\underset{\sim}{\tau}{ }^{\prime} \underset{\sim}{\tau}-\underset{\sim}{\tau}{ }^{\prime} \underset{\sim}{\tau}+2 \underset{\sim}{\tau}{ }^{\prime} \underset{\sim}{\beta}+\underset{\sim}{\beta}{ }^{\prime} \underset{\sim}{\beta} \\
& {[3.8 \cdot 3 . b]}
\end{aligned}
$$

Voltando em [3.8.3.a], segue-se que:

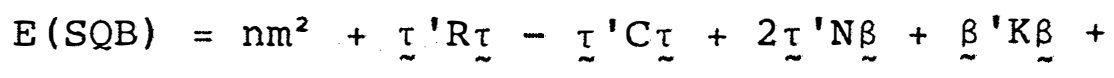

$$
\begin{aligned}
& +\operatorname{TR}\left[X_{3} K^{-1} X_{3}^{\prime} E\left(\underset{\sim}{\varepsilon} \varepsilon^{\prime}\right)\right]-n m^{2}-\sigma^{2} .
\end{aligned}
$$




$$
\text { Mas, } E\left(\underset{\sim}{\varepsilon \varepsilon^{\prime}}\right)=\sigma^{2} I \text {, e } T R\left(X_{3} K^{-1} X_{3}^{\prime}\right)=T R\left(I_{b}\right)=b \text {; }
$$

logo,

$$
E(S Q B)=I^{\prime} R_{\sim}-\underline{\sim}^{\prime} C_{\sim}+2 \underset{\sim}{\tau}{ }^{N}{ }_{\sim}^{\beta}+\underline{\sim}^{\prime} K_{\sim}^{\beta}+(b-1) \sigma^{2}
$$

3.8 .4 - Esperança matemática de $\underset{\sim}{y} \underline{\sim}^{y}-C_{0}$

A soma de quadrados devida ao total é:

$$
\begin{aligned}
& \mathrm{SQT}=\underset{\sim}{\mathrm{Y}^{\prime}} \underset{\sim}{\mathrm{Y}}-\mathrm{C}_{0} \\
& =\left(\underline{\sim}^{\prime} X^{\prime}+\underline{\sim}^{\prime}\right)(X \underset{\sim}{\theta}+\underset{\sim}{\varepsilon})-C_{0} .
\end{aligned}
$$

Tem-se, então, que:

$$
\begin{aligned}
\mathrm{E}(\mathrm{SQT}) & =\underline{\sim}^{\prime} \mathrm{X}^{\prime} \mathrm{X} \underset{\sim}{\theta}+\mathrm{E}\left(\underline{\sim}^{\prime} \underline{\sim}^{\varepsilon}\right)-\mathrm{E}\left(\mathrm{C}_{0}\right) \\
& =\underline{\sim}^{\prime} \mathrm{X}^{\prime} \mathrm{X} \underset{\sim}{\theta}+\mathrm{TR}\left[\mathrm{E}\left(\underset{\sim}{\varepsilon} \underline{\sim}^{\prime}\right)\right]-\mathrm{nm}^{2}-\sigma^{2} \\
& ={\underset{\sim}{\theta}}^{\prime} \mathrm{X}^{\prime} \mathrm{X} \underset{\sim}{\theta}+\mathrm{n}^{2}-\mathrm{nm}^{2}-\sigma^{2} \quad \text { [3.8.4.a] }
\end{aligned}
$$

onde,

$$
\stackrel{\sim}{\theta}^{\prime}=\left[\mathrm{m},{\underset{\sim}{\tau}}^{\prime}, \underset{\sim}{\beta^{\prime}}\right], \quad X^{\prime} X=\left(\begin{array}{ccc}
\mathrm{n} & \underset{\sim}{x^{\prime}} & \underset{\sim}{z^{\prime}} \\
\underset{\sim}{x} & R & N \\
\underset{\sim}{z} & N^{\prime} & k
\end{array}\right) .
$$


Assim,

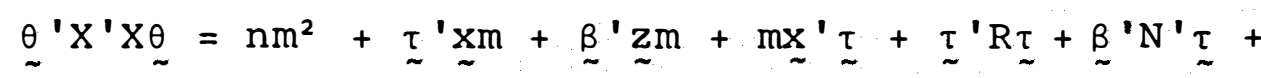

$$
\begin{aligned}
& +\underline{\sim}^{\prime} \dot{\sim}^{\beta}+\underline{\sim}^{\prime} \mathrm{N} \underset{\sim}{\beta}+\underline{\beta^{\prime}} \mathrm{K} \underset{\sim}{\beta} .
\end{aligned}
$$

Impondo-se as restrições usuais, tem-se:

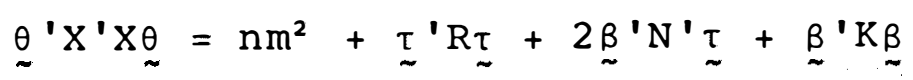

Substituindo-se [3.8.4.b] em [3.8.4.a], segue-se que:

$$
\mathrm{E}(\mathrm{SQT})=\underset{\sim}{\tau}{ }_{\sim}^{\mathrm{R} \tau}+\underset{\sim}{\beta^{\prime}} \mathrm{N}^{\prime} \tau+\underset{\sim}{\beta^{\prime} \mathrm{K}} \underset{\sim}{\beta}+(\mathrm{n}-1) \sigma^{2}[3.8 .4 . \mathrm{C}]
$$

3.8.5 - Esperança matemática da SQResíduo

A soma de quadrados do resíduo é:

$$
\mathrm{SQR}=\mathrm{SQT}-\mathrm{SQTaj} \cdot \mathrm{SQB} \text {. }
$$

Então, por [3.7.2.2.a], [3.7.2.3.c] e [3.7.2.4.b], obtēm-se:

$$
E(S Q R)=\left(n-v^{\prime}-b+1\right) \sigma^{2}
$$




\subsection{6 - Esperança matemática da sQTad.}

A soma de quadrados dos tratamentos adicionais é dada por:

$$
\text { SQTad. }=\frac{1}{b} \underset{s^{\prime}=1}{c} T_{a_{S^{\prime}}}^{c}-\frac{1}{b c}\left(\sum_{s^{\prime}=1}^{c} T_{a_{s^{\prime}}}\right)^{2}[3.8 .6 . a]
$$

Considerando-se a partição $x_{2}^{\prime}=\left[\begin{array}{l}x_{21}^{\prime} \\ \vdots\end{array} x_{22}^{\prime}\right]$, onde $\mathrm{x}_{21}$ é a matriz dos coeficientes associados aos tratamentos originais, de dimensões $\left(b k^{\prime}\right) \times(v), e x_{2}$ a matrizdos coeficientes associados aos tratamentos adicionais, de dimensões $\left(b k^{\prime}\right) \times(c)$, a expressão em [3.8.6.a] pode ser escrita, matricialmente, na seguinte forma:

$$
\text { SQTad. }=\frac{1}{b}\left(x_{22}^{\prime} \underset{\sim}{y}\right)^{\prime}\left(x_{22}^{\prime} \underset{\sim}{y}\right)-\frac{1}{b C}\left(J_{\sim}^{\prime} x_{22}^{\prime} \underset{\sim}{y}\right)^{\prime}\left(\underset{\sim}{J^{\prime}} x_{2}^{\prime} \underset{2}{\underline{y}}\right),
$$

onde J é um vetor de uns de dimensões (c) x(1).

Tem-se então que:

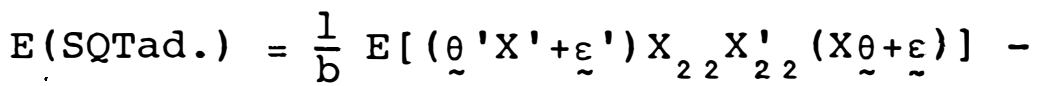

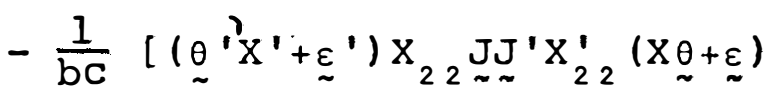

$$
\begin{aligned}
& =\frac{1}{b}\left[\underset{\sim}{\theta} X^{\prime} X_{2.2} X_{22}^{\prime} X_{\sim}^{\theta}+E(\underbrace{\prime}_{\sim} X_{22} X_{22}^{\prime} \underset{\varepsilon}{\varepsilon})\right]-
\end{aligned}
$$

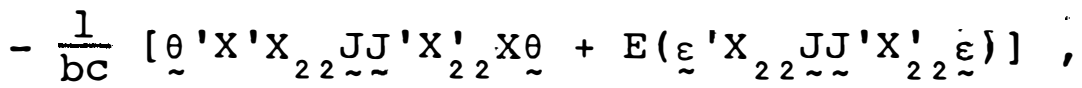




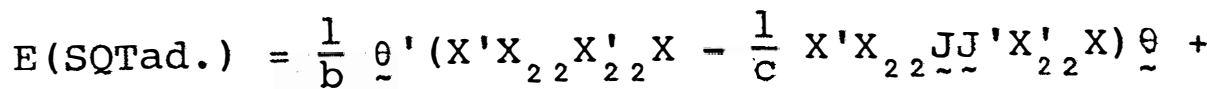

$$
+\frac{1}{b} E\left[\underset{\sim}{\varepsilon^{\prime}}\left(X_{22} X_{22}^{\prime}-\frac{1}{c} X_{22} \underset{\sim}{J} J^{\prime} X_{22}^{\prime}\right) \varepsilon\right],
$$

onde,

$$
x^{\prime} x_{22}=\left(\begin{array}{c}
x_{1}^{\prime} x_{22} \\
x_{22}^{\prime} x_{22} \\
x_{22}^{\prime} x_{22} \\
x_{3}^{\prime} x_{22}
\end{array}\right)=\left(\begin{array}{c}
x_{2}^{\prime} \\
\phi \\
R_{2} \\
N_{2}^{\prime}
\end{array}\right) \text { e } x^{\prime} x_{22} J=\left(\begin{array}{c}
x_{2}^{\prime} J \\
\sim \\
R_{2} J \\
N_{2}^{\prime} J \\
\sim \\
\sim
\end{array}\right)
$$

Pode-se verificar que:

i) $X^{\prime} X_{22} X_{22}^{\prime} X=\left(\begin{array}{cccc}\mathrm{Cb}^{2} & \phi & \underset{\sim}{x_{2}^{\prime}} R_{2} & \underset{\sim}{x} N_{2}^{\prime} \\ \phi & \phi & \phi & \phi \\ R_{2} \underset{\sim}{x} & \phi & R_{2}^{2} & R_{2} N_{2} \\ N_{2}^{\prime} \underset{\sim 2}{X} & \phi & N_{2}^{\prime} R_{2} & N_{2}^{\prime} N_{2}\end{array}\right)$

$[3.8 \cdot 6 . c]$

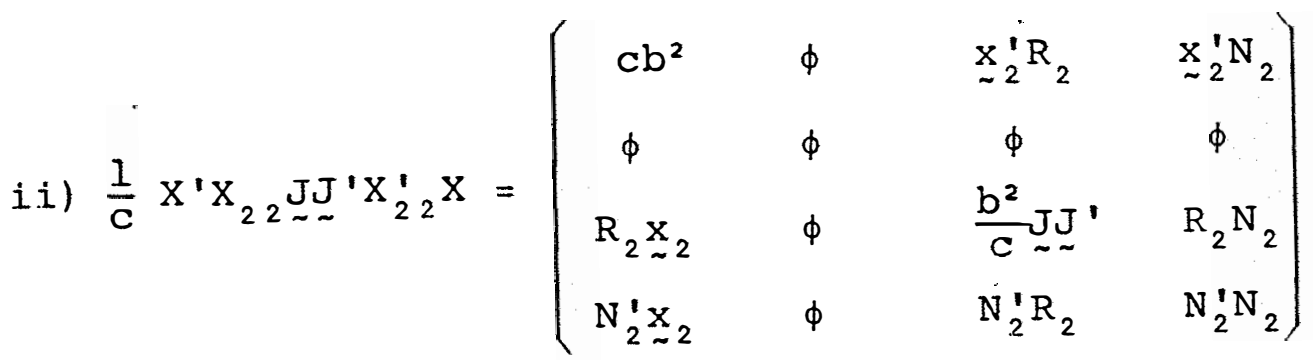

$[3 \cdot 8 \cdot 6 . d]$ 
iii) $\frac{1}{b} E\left(\underset{\sim}{\varepsilon} X_{22} X_{22}^{\prime} \underset{\sim}{\varepsilon}\right)=\frac{1}{b} E\left[T R\left(X_{22} X_{22 \underset{\sim}{\varepsilon} \underset{\sim}{\varepsilon}}^{\prime}\right)\right]$

$$
\begin{aligned}
& =\frac{I}{b} \operatorname{TR}\left[X_{22} X_{22}^{\prime} E\left(\sim_{\sim}^{\varepsilon}{ }^{\prime}\right)\right] \\
& =c \sigma^{2}
\end{aligned}
$$

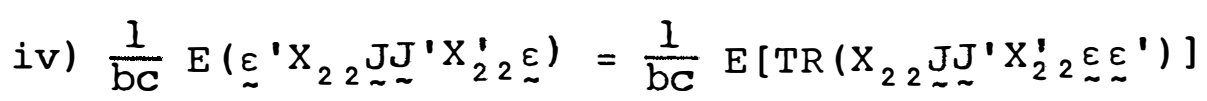

$$
\begin{aligned}
& =\frac{1}{\mathrm{bC}} \operatorname{TR}\left[\mathrm{X}_{2} \underset{2}{\mathrm{JJ}} \sim_{\sim}^{\prime} \mathrm{X}_{22}^{\prime} \mathrm{E}\left(\underset{\sim}{\varepsilon \varepsilon^{\prime}}\right)\right] \\
& =\sigma^{2}
\end{aligned}
$$

Considerando-se as expressões de (i), (ii), (iii) e (iv) em [3.6.6.b], tem-se então que:

$$
E(\text { SQTad. })=\underset{\sim 2}{\tau_{2}^{\prime}}\left(R_{2}-\frac{b}{c} \underset{\sim}{J J} J^{\prime}\right) \tau_{-2}+(c-1) \sigma^{2} \quad[3.8 .6 . g]
$$

\subsection{7 - Esperança matemática da SQTipos}

Sabe-se que a soma de quadrados entre os dois tipos de tratamentos é dada por:

$$
\text { SQTipos }=\frac{1}{b k}\left(\sum_{S=1}^{v} T_{S^{\prime}}\right)^{2}+\frac{1}{b c}\left(\underset{s^{\prime}=1}{c} T_{a_{S^{\prime}}}\right)^{2}-\frac{G^{2}}{b k^{\prime}},
$$

ou, matricialmente, 


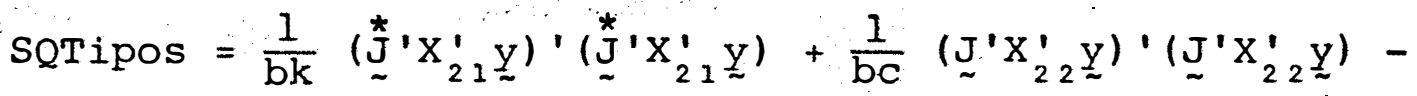

$$
\begin{aligned}
& \text { - E(SQm) , }
\end{aligned}
$$

onde $\stackrel{*}{J}$ é um vetor de uns de dimensões $(v) \times(1)$.

Tem-se, então, que:

$$
\begin{aligned}
& E(\text { SQTipos })=\frac{1}{b k} E\left[\left(\underline{\sim}^{\prime} X^{\prime}+\varepsilon^{\prime}\right) X_{21} \underset{\sim}{J} \sim_{\sim}^{*} X_{21}^{\prime}\left(X_{\sim}^{\theta}+\underset{\sim}{\varepsilon}\right)\right]+
\end{aligned}
$$

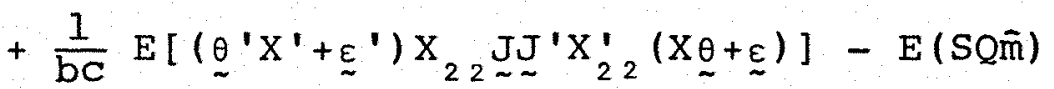

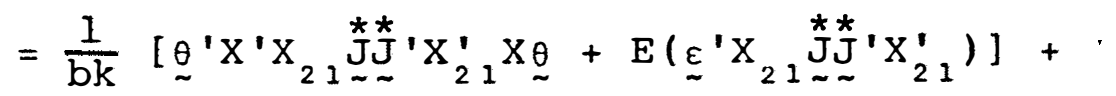

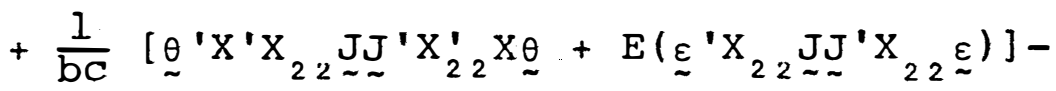

$$
\begin{aligned}
& \text { - E (SQmin) }
\end{aligned}
$$

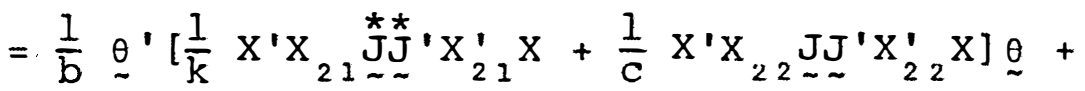

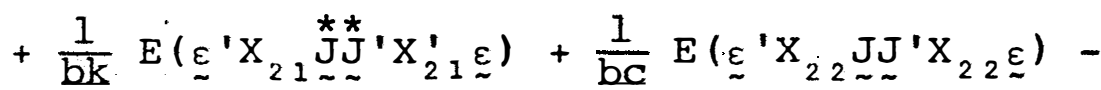

$$
\begin{aligned}
& \text { - E (SQm) } \\
& {[3,8,7 . a]}
\end{aligned}
$$

onde,

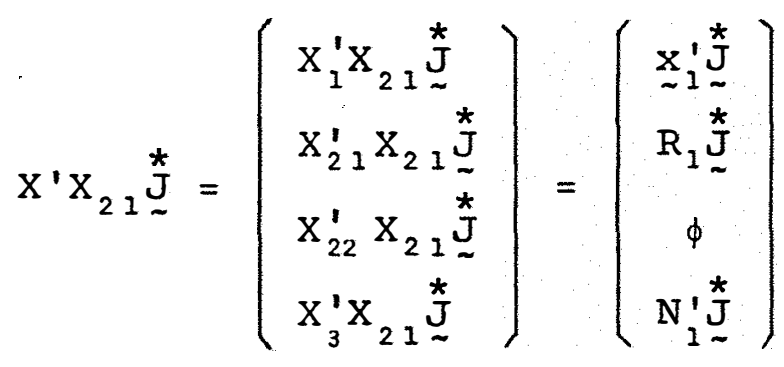


e, portanto,

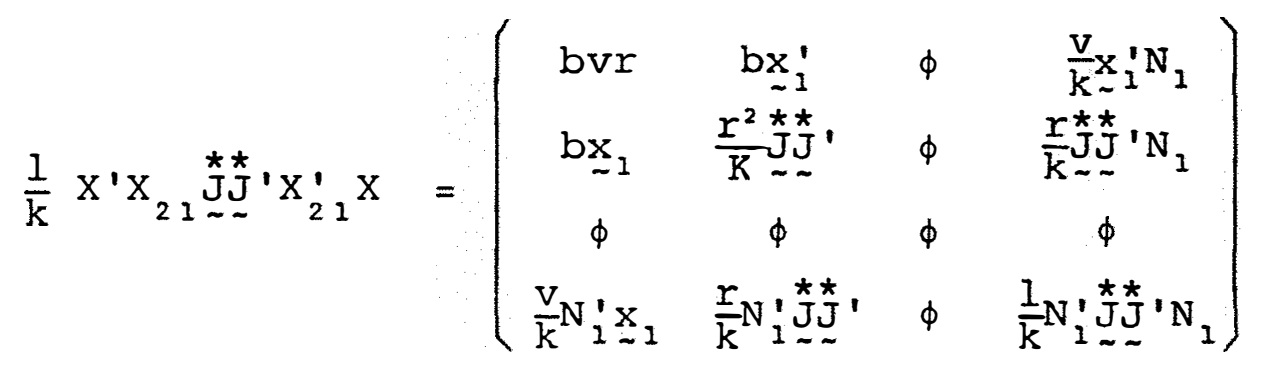

$[3.8 .7 . b]$

Considerando-se as matrizes de [3.8.6.d] e [3.8.7.b], verifica-se que a primeira expressão de E(SQTipos) é:

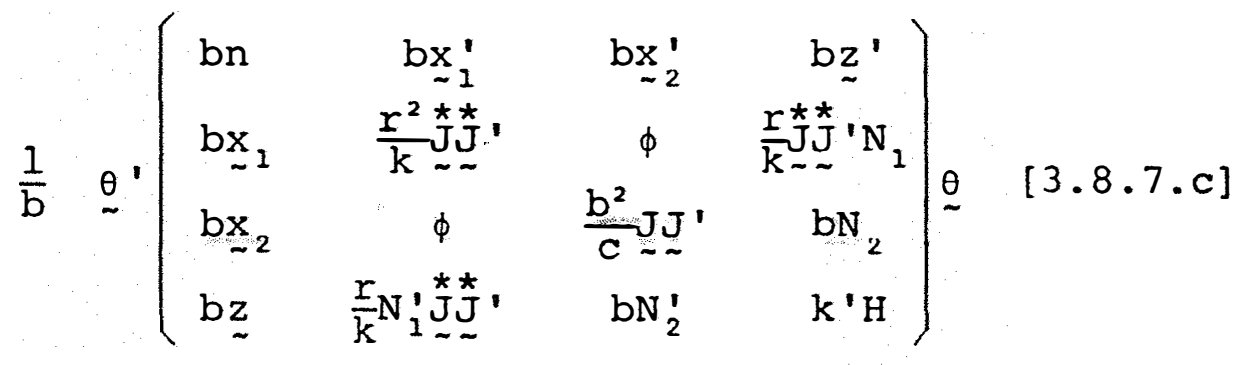

onde $\mathrm{H}$ é uma matriz unitária, de dimensões (b)x(b).

Voltando em [3.8.7.a], tem-se então:

$$
\begin{aligned}
& \mathrm{E}(\mathrm{SQTipos})=\mathrm{nm}^{2}+\underline{\tau}_{1}^{\prime} \underline{\sim}_{1} \mathrm{~m}+\tau_{2}^{\prime} \mathrm{x}_{2} \mathrm{~m}+\underline{\beta}^{\prime} \mathrm{zm}+
\end{aligned}
$$

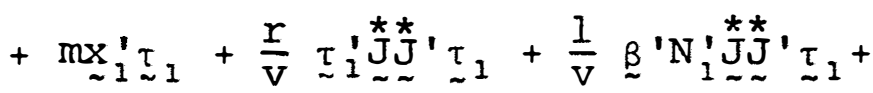

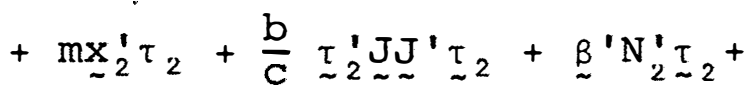

$$
\begin{aligned}
& +m z_{\sim}^{\prime} \underset{\sim}{\beta}+\frac{1}{V} \tau_{I}^{\prime} \mathcal{\sim}_{\sim}^{*} J^{*} N_{I} \underset{\sim}{\beta}+\tau_{2}^{\prime} N_{2} \underset{\sim}{\beta}+
\end{aligned}
$$




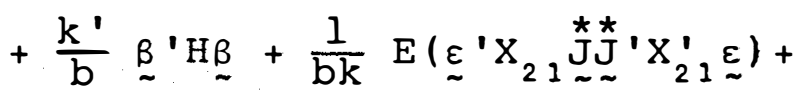

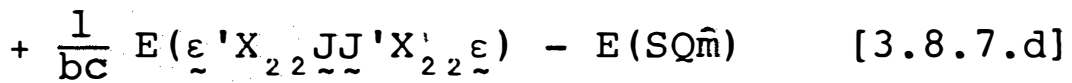

Impondo-se as restrições usuais, e considerando-se $[3.8 .1 . c]$ e $[3.8 .6 . f]$, a expressão [3.8.7.d] fica:

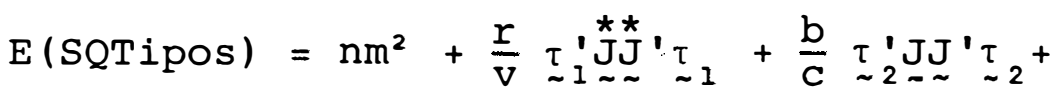

$$
\begin{aligned}
& +\frac{1}{b k} E\left(\varepsilon^{\prime} X_{21} \underset{\sim}{\stackrel{*}{J} J} X_{21}^{\prime} X_{\sim}^{\prime}\right)+\sigma^{2}-n m^{2}-\sigma^{2}
\end{aligned}
$$

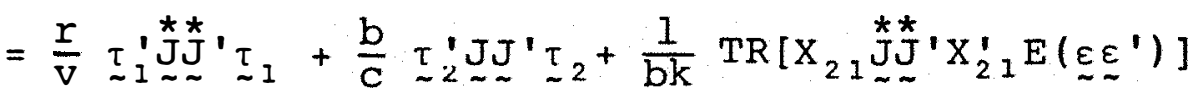

$$
\begin{aligned}
& \text { Mas, } E\left(\underset{\sim}{\varepsilon \varepsilon^{\prime}}\right)=\sigma^{2} I, e \operatorname{TR}\left(X_{21}{\underset{\sim}{J \sim}}_{\sim}^{*}{ }^{*} X_{21}^{\prime}\right)=r v=b k \text {; }
\end{aligned}
$$

logo,

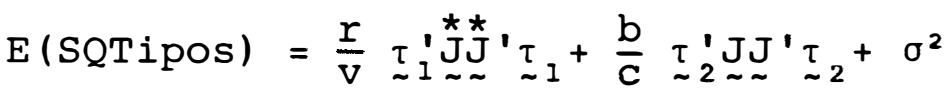

$$
\begin{aligned}
& {[3.8 \cdot 7 . e]}
\end{aligned}
$$

3.8.8 - Esperança matemática da SQTor.(aj.)

Por $[3.7 . n]$ sabe-se que:

SQTaj. = SQTad. + SQTipos + SQTor.(aj.) .

Logo, tem-se: 


$$
\begin{aligned}
& \text { E[SQTor. (aj.)] = E(SQTaj.) - E(SQTad.) }-E \text { (SQTipos) } \\
& =\tau_{\sim}^{\prime} C_{\sim}^{\tau}+\left(V^{\prime}-1\right) \sigma^{2}-\underset{\sim}{\tau}\left(R_{2}-\frac{b}{C} \underset{\sim}{J} \mathcal{I}^{\prime}\right) \tau_{2}-
\end{aligned}
$$

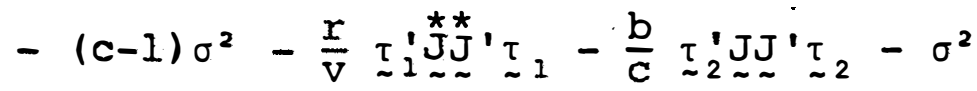

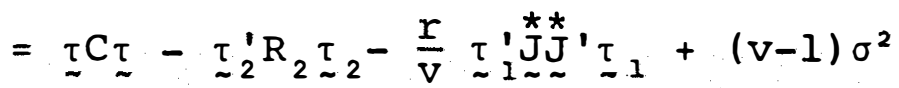$$
\text { [3.8.8.a] }
$$

Pode-se verificar que:

$$
\begin{aligned}
\sim_{\sim}^{\prime} C_{\sim}= & \tau_{1}^{\prime}\left(R_{1}-\frac{1}{k^{\prime}} N_{1} N_{1}^{\prime}\right) \underset{\sim}{\tau}-\frac{1}{k^{\prime}} \tau_{1}^{\prime} N_{1} N_{2}^{\prime} \tau_{2}-\frac{1}{k^{\prime}} \tau_{2}^{\prime} N_{2} N_{1}^{\prime} \tau_{1}+ \\
& +\tau_{2}^{\prime}\left(R_{2}-\frac{1}{k^{\prime}} N_{2} N_{2}^{\prime}\right)_{\sim 2}^{\tau}
\end{aligned}
$$

Assim, a E[SQTor.(aj.)] fica:

$$
\begin{aligned}
E[\text { SQTor. }(a j .)]= & \tau_{-1}^{\prime}\left(R_{1}-\frac{r}{v}{\underset{\sim}{J} J}_{\sim}^{*}\right) \tau_{1}-\frac{1}{k^{\prime}} \tau^{\prime} N^{\prime} \tau+ \\
& +(v-1) \sigma^{2}+
\end{aligned}
$$


3.9 - Distribuição das Formas Quadráticas

Para determinar-se a distribuição das formas quadráticas apresentadas em (3.7), os seguintes teoremas são utilizados:

TEOREMA 1. (GRAYBILL, 1961):

Se $\underline{\sim} \sim N\left(\underline{\sim}, \sigma^{2} I\right)$, então $\underline{y}^{\prime} A \underline{\sim} \sim x^{2}(n, \delta)$ ou se ja, $\underline{\sim}^{\prime} A \underline{\sim}$ tem distribuição de qui-quadrado não central, com n graus de liberdade, e parâmetro de não centralidade

$$
\delta=\underline{\mu}{ }^{\prime}{ }^{\mu} / 2 \sigma^{2},
$$

se e somente se A for uma matriz idempotente de caracteristica $n$.

TEOREMA 2 (SEARLE, 1971 ):

Quando $\underset{\sim}{\mathrm{y}} \sim \mathrm{N}(\underset{\sim}{\mu}, \mathrm{V})$, as formas quadráticas $\underset{\sim}{\mathrm{y}}{ }^{\prime} \mathrm{Ay}$ e $\underset{\sim}{y}$ 'By são distribuídas independentemente, se e somente se $\mathrm{AVB}=\phi$, ou, de forma equivalente, $\mathrm{BVA}=\phi$.

TEOREMA 3 (GRAYBILL, 1961 ):

Se uma variável aleatória w é distribuída c으 mo $x_{\left(n_{1}, \delta\right)}^{2}$ ou seja, como qui-quadrado não central com $n_{1}$ graus de liberdade e parâmetro $\delta$, e se outra variável aleatória $z$ é distribuída como $x^{2}\left(\mathrm{n}_{2}\right)$, ou seja, como qui-quadra- 
do central com $\mathrm{n}_{2}$ graus de liberdade, e se $\mathrm{w}$ e $z$ são independentes, então a quantidade

$$
\mathrm{u}=\frac{\mathrm{wn}}{\mathrm{zn_{1 }}}
$$

tem distribuição $\mathrm{F}$ não central $\operatorname{com} \mathrm{n}_{1}$ e $\mathrm{n}_{2}$ graus de liberda de, e parâmetro de não centralidade $\delta$.

$$
3.9 .1 \text { - Distribuição da SQTaj./o }
$$

Sabe-se que a sQTaj. é:

$$
\underline{\tau}^{\prime} \underset{\sim}{Q}=\underline{Q}^{\prime} M^{-1} \underline{Q}=\underline{Y}^{\prime} A_{1} \underline{\underline{Y}}
$$

onde $A_{1}=X W^{\prime} M^{-1} W^{\prime}$.

$$
\text { Pode-se verificar que: }
$$

$$
A_{1}^{2}=X W^{\prime} M T^{-1} W X^{\prime} X W^{\prime} M{ }^{-1} W X ' \text {. }
$$

Mas, por $[3.6 .1 . b]$, chega-se a $W^{\prime} X W=c ; 10$ go,

$$
\begin{aligned}
A_{1}^{2} & =X W^{\prime} M{ }^{-1} C M^{-1} W X \\
& =X W^{\prime} M{ }^{-1}\left(A^{\prime}+M^{\prime}\right) M{ }^{-1} W X \\
& =X W^{\prime} M{ }^{-1} A^{\prime}+X W^{\prime} M^{-1} W X^{\prime},
\end{aligned}
$$

que, por $[3.6 .2 . \ell]$, fica:

$$
A_{1}^{2}=X W^{\prime} M{ }^{-1} W X^{\prime}=A_{1},
$$


ou seja, $A_{1}$ é uma matriz idempotente; e ainda,

$$
\begin{aligned}
\operatorname{Caract} \cdot\left(A_{1}\right) & =\operatorname{TR}\left(X W^{\prime} M^{-1} W X^{\prime}\right) \\
& =\operatorname{TR}\left(M^{-1} W X^{\prime} X W^{\prime}\right) \\
& =\operatorname{TR}\left(M^{-1} C\right) \\
& =\operatorname{TR}(C)=V^{\prime}-1
\end{aligned}
$$

Tem-se ainda:

$$
\underline{\mu}^{\prime} A_{I} \underset{\sim}{\mu}=\underline{\theta}^{\prime} X^{\prime} X W W^{\prime} M T^{l} W X^{\prime} X \underline{\theta},
$$

que, por $[3.6 .1 . b]$ e [3.6.2.j], fica:

$$
\underline{\sim}^{\prime} \mathrm{A}_{1} \underset{\sim}{\mu}=\underline{\tau}^{\prime} \mathrm{C} \underline{\tau}
$$

Assim, pelo Teorema 1, tem-se:

$$
\bar{\tau}^{\prime} Q \sim x^{2}\left(n_{1}, \delta_{1}\right),
$$

onde:

$$
\begin{aligned}
& \mathrm{n}_{1}=v^{\prime}-1 ; \\
& \delta_{1}=\frac{1}{2 \sigma^{2}} \tau^{\prime} \mathrm{C} \tau .
\end{aligned}
$$


A SQTad. pode ser colocada na forma:

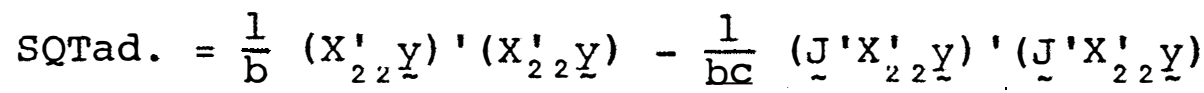

$$
\begin{aligned}
& =\underline{\sim}^{\prime} A_{2} \underline{y},
\end{aligned}
$$

onde,

$$
\begin{aligned}
& A_{2}=x_{22} R_{2}^{-1} x_{22}^{\prime}-x_{22} \frac{J J^{\prime}}{b c} x_{22}^{\prime} \cdot \\
& \text { Verifica-se que: } \\
& A_{2}^{2}=X_{22} R_{2}^{-1} X_{22}^{\prime} x_{22} R_{2}^{-1} X_{22}^{\prime}-x_{22} R_{2}^{-1} x_{22}^{\prime} x_{22} \frac{J J^{\prime}}{b c} x_{22}^{\prime}- \\
& -x_{22} \frac{\underset{J}{J} J^{\prime}}{b c} x_{22}^{\prime} x_{22} R_{2}^{-1} x_{22}^{\prime}+x_{22} \frac{J^{\prime} J^{\prime}}{b c} x_{22}^{\prime} x_{22} \frac{J^{J} J^{\prime}}{b c} x_{22}^{\prime} \\
& =\mathrm{X}_{22} \mathrm{R}_{2}^{-1} \mathrm{R}_{2} \mathrm{R}_{2}^{-1} \mathrm{X}_{22}^{\prime}-\mathrm{x}_{22} \mathrm{R}_{2}^{-1} \mathrm{R}_{2} \frac{\mathrm{JJU}^{\prime}}{\mathrm{bc}} \mathrm{x}_{22}^{\prime}- \\
& -x_{22} \frac{J^{J} J^{\prime}}{b c} R_{2} R_{2}^{-1} x_{22}^{\prime}+x_{22} \frac{J^{J} J^{\prime}}{b c} R_{2} \frac{J^{\prime} J^{\prime}}{b c} x_{22}^{\prime} \\
& =x_{22} R_{2}^{-1} x_{22}^{\prime}-x_{22} \frac{\underset{J J}{J}{ }^{\prime}}{b c} x_{22}^{\prime}-\frac{x_{222}^{J J}{ }^{\prime} x_{22}^{\prime}}{b c}+ \\
& +x_{22} \frac{J^{J} J^{\prime}}{b c} R_{2} \frac{J^{J} J^{\prime}}{b c} x_{22}^{\prime}
\end{aligned}
$$$$
\text { Como } \underset{\sim}{J} R_{2} \underset{\sim}{J}=b c \text {, segue-se: }
$$$$
\mathrm{A}_{2}^{2}=\mathrm{X}_{22} \mathrm{R}_{2}^{-1} \mathrm{X}_{22}^{\prime}-\mathrm{X}_{22} \frac{\mathrm{J} \mathrm{J}^{\prime}}{\mathrm{bc}} \mathrm{X}_{22}^{\prime}=\dot{A}_{2},
$$ 
ou seja, $A_{2}$ é uma matriz idempotente; e ainda:

$$
\begin{aligned}
\text { Caract. }\left(A_{2}\right) & =\operatorname{TR}\left(x_{22}^{\prime} x_{22} R_{2}^{-1}-x_{22}^{\prime} x_{22} \frac{J^{\prime}}{b c}\right) \\
& =\operatorname{TR}\left(R_{2} R_{2}^{-1}-R_{2} \frac{J^{\prime}}{b c}\right) \\
& =\operatorname{TR}\left[I_{C}-I\right]=C-I .
\end{aligned}
$$

Tem-se também:

$$
\underline{\sim}^{\prime} A_{2} \underset{\sim}{\mu}=\underline{\theta}^{\prime} X^{\prime}\left(x_{22} R_{2}^{-1} x_{22}^{\prime}-x_{22} \frac{J J^{\prime}}{b c} x_{22}^{\prime}\right) x_{\sim}^{\theta},
$$

que, por $[3.8 .6 . c]$ e [3.8.6.d], fica:

$$
\underline{\mu}^{\prime} A_{2} \underset{\sim}{\mu}=\tau_{2}^{\prime}\left(R_{2}-\frac{b}{C} \underset{\sim}{J}{ }^{\prime}\right) \tau_{2}
$$

Assim, pelo Teorema 1, tem-se:

$$
\operatorname{SQTad} . / \sigma^{2} \backsim x^{2}\left(\mathrm{n}_{2}, \delta_{2}\right)
$$

onde:

$$
\begin{aligned}
& \mathrm{n}_{2}=c-1 ; \\
& \delta_{2}=\frac{1}{2 \sigma^{2}} \underline{\sim}_{2}^{\prime}\left(R_{2}-\frac{b}{c} \underset{\sim}{J} \sim^{\prime}\right) \underset{\tau_{2}}{\tau} .
\end{aligned}
$$


3.9.3 - Distribuição da SQTipos/ $/ \sigma^{2}$

A SQTipos é dada por:

$$
\begin{aligned}
& \text { SQTipos }=\frac{1}{\mathrm{bk}}\left(\stackrel{\sim}{J}^{*} X_{21}^{\prime} \underset{\sim}{y}\right) \cdot\left(\stackrel{\sim}{J}^{*} X_{21}^{\prime} \underset{\sim}{y}\right)+\frac{1}{\mathrm{bC}}\left(\underline{J}^{\prime} X_{22}^{\prime} \underset{\sim}{y}\right) \cdot\left(\mathcal{\sim}^{\prime} X_{22}^{\prime} \underset{\sim}{y}\right)- \\
& -\frac{1}{n}\left(x_{i}^{\prime} \underset{\sim}{y}\right) \cdot\left(x_{i}^{\prime} \underset{\sim}{y}\right) \\
& =\underline{\mathrm{y}}^{\prime} \mathrm{A}_{3} \underline{\mathrm{y}},
\end{aligned}
$$

onde,

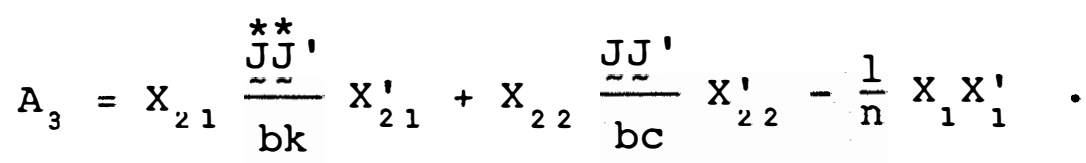

$$
\begin{aligned}
& \text { Verifica-se que: }
\end{aligned}
$$

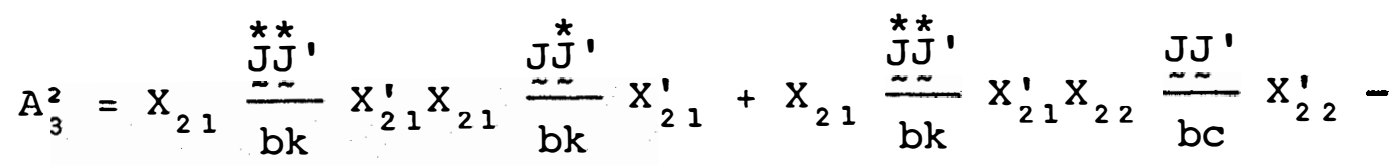

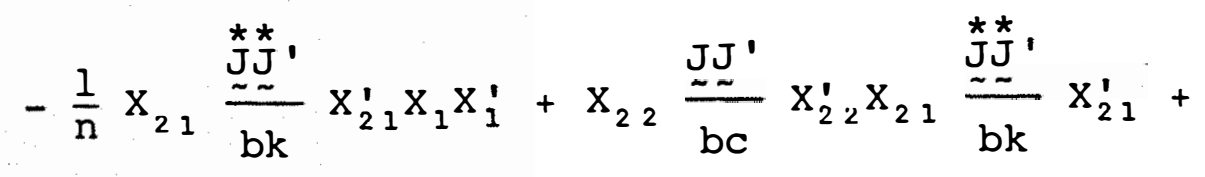

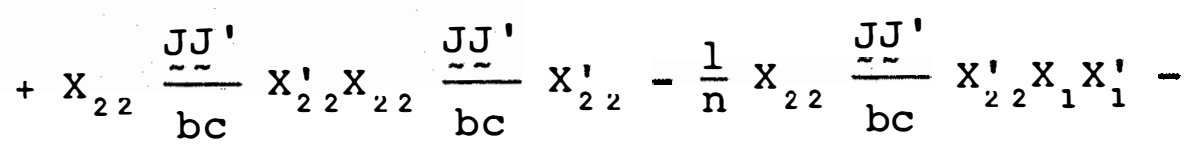

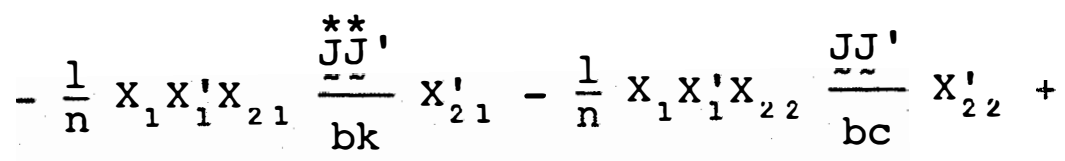

$$
\begin{aligned}
& +\frac{1}{n^{2}} x_{1} x_{1}^{\prime} x_{1} x_{1}^{\prime}
\end{aligned}
$$


Mas,

$$
\begin{aligned}
& \mathrm{bk}=r \mathrm{v}, \\
& \stackrel{\star}{J}{ }_{\sim}^{\prime} x_{21}^{\prime} x_{21} \stackrel{\star}{J}=\stackrel{*}{J} \cdot R_{1} \stackrel{\star}{J}=r v, \\
& x_{21}^{\prime} x_{22}=\phi \text {, } \\
& \underline{J}^{\prime} X_{22}^{\prime} X_{22} \underset{\sim}{J}=J_{\sim}^{\prime} R_{2} \underset{\sim}{J}=b c \text {, } \\
& x_{1}^{\prime} x_{1}=n \text {; }
\end{aligned}
$$

logo, tem-se:

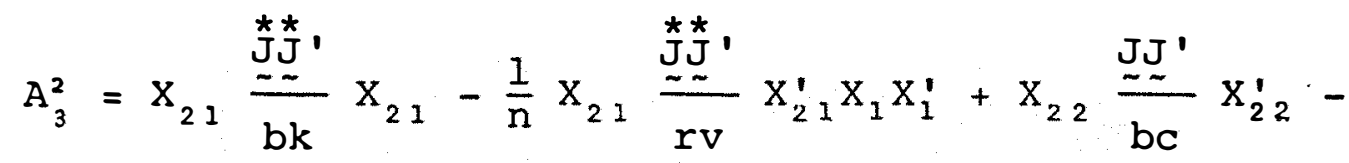

$$
\begin{aligned}
& -\frac{1}{n} x_{22} \frac{J J^{\prime}}{b c} x_{22}^{\prime} x_{1} x_{1}^{\prime}-\frac{1}{n} x_{1} x_{1}^{\prime} x_{21} \frac{{\stackrel{\star N}{J} J^{\prime}}^{\sim}}{r v} x_{21}^{\prime}- \\
& -\frac{1}{n} x_{1} x_{1}^{\prime} x_{22} \frac{J J^{\prime}}{b c} x_{22}^{\prime}+\frac{1}{n} x_{1} x_{1}^{\prime} \\
& \text { Pode-se, verificar ainda que } x_{2}^{\prime} x_{1}=r \stackrel{\star}{J},
\end{aligned}
$$

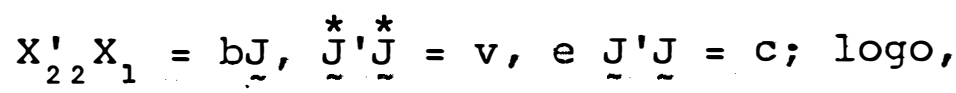

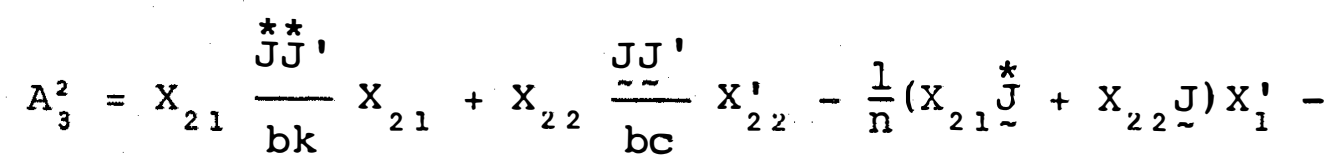

$$
\begin{aligned}
& -\frac{1}{n} x_{1}\left(\stackrel{*}{J^{\prime}} x_{21}^{\prime}+\underset{\sim}{J} x_{22}\right)+\frac{1}{n} x_{1} x_{1}^{\prime}
\end{aligned}
$$


Mas, $x_{21} \stackrel{\star}{J}+x_{22} \underset{J}{J}=x_{1}$; portanto,

$$
A_{3}^{2}=x_{21} \frac{{\stackrel{\star J}{J} J^{\prime}}^{\prime}}{b k} x_{21}^{\prime}+x_{22} \frac{J^{J} J^{\prime}}{b c} x_{22}^{\prime}-\frac{1}{n} x_{1} x_{1}^{\prime}=A_{3},
$$

ou seja, $A_{3}$ é uma matriz idempotente; e ainda:

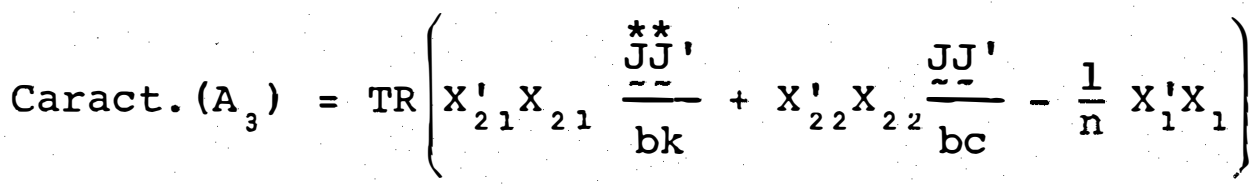

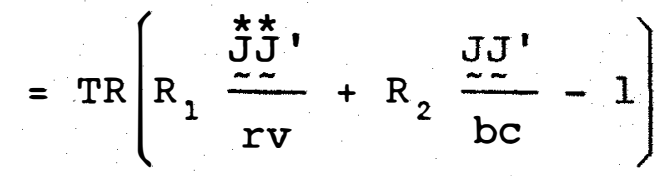

$$
\begin{aligned}
& =\operatorname{TR}\left(\frac{\substack{* * \\
\mathcal{J} J}}{v}+\frac{J J^{\prime}}{c}-1\right)=1 .
\end{aligned}
$$

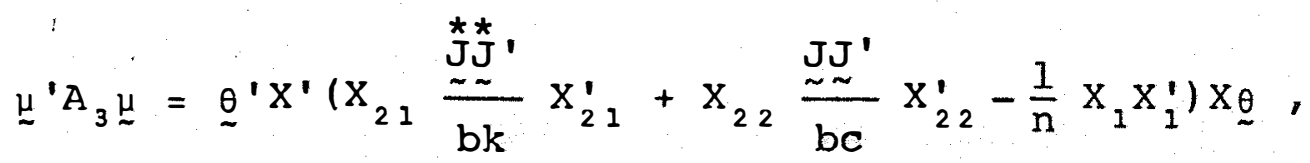

que, por $[3.8 .7 . c]$ e $[3.8 .1 . b]$, fica:

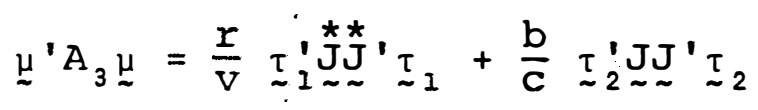

Assim, pelo Teorema 1, tem-se:

$$
\text { SQTipos } / \sigma^{2} \sim x_{\left(n_{3}, \delta_{3}\right),}^{2} \quad[3.9 .3 . d]
$$

onde:

$$
\begin{aligned}
& \mathrm{n}_{3}=1 \\
& \delta_{3}=\frac{1}{2 \sigma^{2}}\left(\frac{r}{v} \tau_{1}^{\prime} J_{\sim \sim}^{*} J^{*} \tau_{1}+\frac{b}{c}{\underset{\sim}{\prime}}_{2}^{\prime} J J^{\prime} \tau_{2}\right) .
\end{aligned}
$$


3.9.4 - Distribuição da SQTor. (aj.)/ $/ \sigma^{2}$

A SQTor.(aj.) é:

SQTor.(aj.) = SOTaj. - SQTad. - SQTipos,

que por $[3.7 . e],[3.7 . i]$ e [3.7.l], fica:

$$
\begin{aligned}
& \text { SQTor. (aj.) = I' } \tau_{\sim}-\frac{1}{b} \underset{s^{\prime}=1}{c} Q_{a_{s^{\prime}}}^{2}+\frac{1}{b c}\left(\sum_{s^{\prime}=1}^{c} Q_{a_{s^{\prime}}}\right)^{2}- \\
& -\frac{k^{\prime}}{\operatorname{rvc}}\left(\sum_{s=1}^{v} Q_{s}\right)^{2}
\end{aligned}
$$

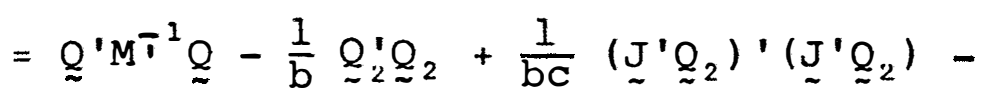

$$
\begin{aligned}
& -\frac{k^{\prime}}{\operatorname{rvc}}\left(\stackrel{\sim}{J}^{\prime} \underline{\sim}_{1}\right) \cdot\left(\stackrel{*}{J}^{\prime} \stackrel{\sim}{2}_{1}\right) \\
& =\underline{Z}^{\prime} M^{\top}{ }^{-1} \underset{\sim}{Q}-\frac{1}{b}{\underset{\sim}{2}}_{2}^{\prime}{\underset{\sim}{2}}_{2}+\frac{1}{\mathrm{bC}}{\underset{\sim}{2}}_{2}^{\prime} \mathrm{JJ}_{\sim}^{\prime} \underline{\sim}_{2}-
\end{aligned}
$$

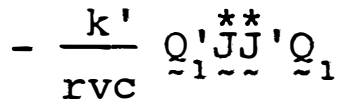

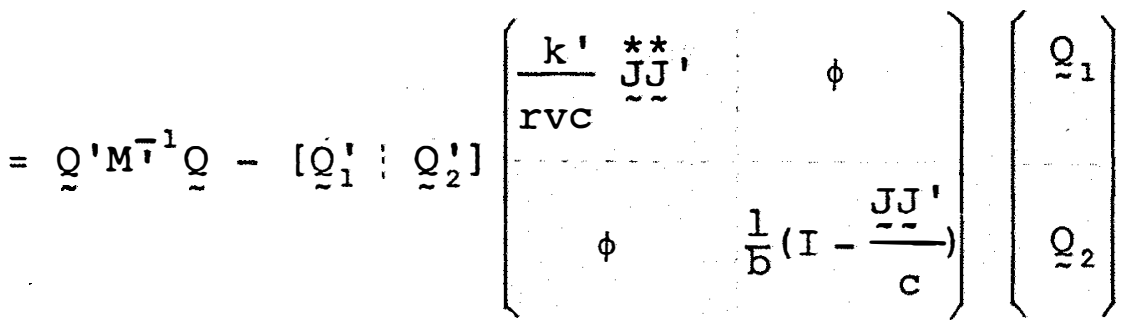

$$
\begin{aligned}
& =\mathscr{\sim}^{\prime}\left(M^{-1}-D\right) \underset{\sim}{Q},
\end{aligned}
$$


onde:

$$
D=\left(\begin{array}{ccc}
\frac{k^{\prime}}{r v c}{\stackrel{\sim}{J} J^{*}}^{*} & \\
\phi & \frac{1}{b}\left(I-\frac{J J^{\prime}}{c}\right)
\end{array}\right)
$$

Como $\underset{\sim}{Q}=W^{\prime} \underline{\sim}$, tem-se que:

$$
\begin{aligned}
\text { SQTOr. (aj.) } & =\underline{y^{\prime}} X W^{\prime}\left(M^{-1}-D\right) W X^{\prime} \underline{y} \\
& =\underline{y}^{\prime} A_{4} \underset{\sim}{y}
\end{aligned}
$$

onde, $A_{4}=X W^{\prime} M^{-1} W X^{\prime}-X W^{\prime} D W X '$.

Verifica-se que:

$$
\begin{aligned}
A_{4}^{2}= & X W^{\prime} M^{-1} W X^{\prime} X W^{\prime} M^{-1} W X^{\prime}+X W^{\prime} D W X^{\prime} X W^{\prime} D W X^{\prime}- \\
& -X W^{\prime} M^{-1} W X^{\prime} X W^{\prime} D W X^{\prime}-X W^{\prime} D W X^{\prime} X W^{\prime} M^{-1} W X^{\prime} \\
& \text { Mas, por }[3.6 .1 . b], W X^{\prime} X W^{\prime}=C ; \text { logo, } \\
A_{4}^{2}= & X W^{\prime} M^{-1} C M^{-1} W X^{\prime}+X W^{\prime} D C D W X^{\prime}-X W^{\prime} M^{-1} C D W X '- \\
& -X W^{\prime} D C M{ }^{-1} W X^{\prime} .
\end{aligned}
$$

Sabe-se que $C=M^{\prime}+A^{\prime} ;$ pode-se verificar tam bèm que $D C D=D$. Assim,

$$
\begin{aligned}
A_{4}^{2}= & X W^{\prime} M{ }^{-1} W X^{\prime}+X W^{\prime} M^{-1} A^{\prime} M^{-1} W X^{\prime}+X W^{\prime} D W X^{\prime}-X W^{\prime} D W X^{\prime}- \\
& -X W^{\prime} M T^{-1} A^{\prime} D W X^{\prime}-X W^{\prime} D W X^{\prime}-X W^{\prime} D A M^{\prime} I^{-1} W X^{\prime} .
\end{aligned}
$$


Mas, por $[3.6 .2 . \ell], X W^{\prime} M^{-1} A^{\prime}=\phi ;$ e $A^{\prime} M^{-1} W X^{\prime}=$ $=\phi$, tomando-se uma matriz A simétrica. Segue-se então que:

$$
A_{4}^{2}=X W^{\prime} M^{-1} W X^{\prime}-X W^{\prime} D W X^{\prime}=A_{4},[3.9 .4 . d]
$$

ou seja, $\mathrm{A}_{4}$ è uma matriz idempotente; e ainda,

$$
\begin{aligned}
\text { Caract. }\left(A_{4}\right) & =\operatorname{TR}\left(X W^{\prime} M{ }^{-1} W X^{\prime}-X W^{\prime} D W X^{\prime}\right) \\
& =\operatorname{TR}\left(M{ }^{-1} W X^{\prime} X W^{\prime}\right)-T R\left(D W X^{\prime} X W^{\prime}\right) \\
& =\operatorname{TR}\left(M{ }^{-1} C\right)-T R(D C) \\
& =\text { Caract. }(C)-T R(D C) .
\end{aligned}
$$

Pode-se verificar que:

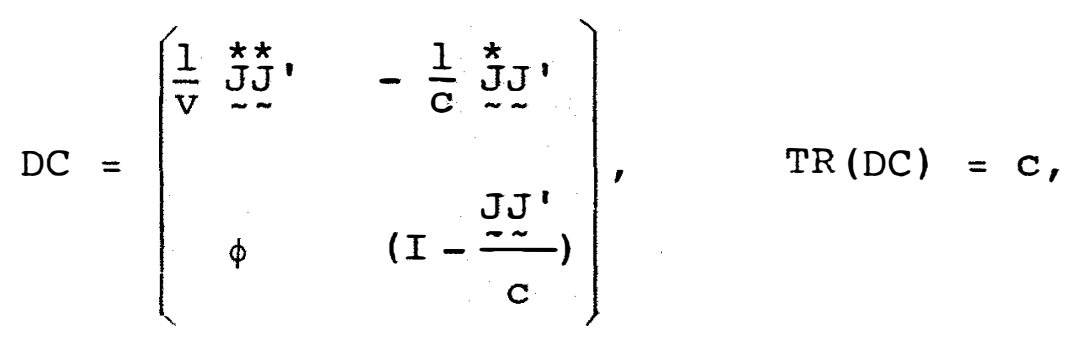

Logo, como Caract. (C) $=v^{\prime}-1=v+C-1$, tem-se:

$$
\begin{aligned}
& \text { Caract. }\left(A_{4}\right)=v+c-1-c=v-1 \\
& \underline{\sim}^{\prime} A_{4} \underset{\sim}{\mu}=\underline{\sim}^{\prime} X^{\prime}\left(X W^{\prime} M^{-1} W X^{\prime}-X W^{\prime} D W X^{\prime}\right) X \underset{\sim}{\theta} \\
& =\tau^{\prime} \mathrm{CM}^{-1} \mathrm{C} \underset{\sim}{\tau}-\tilde{\sim}^{\prime} \mathrm{CDC} \underset{\sim}{\tau} \\
& =\underline{\tau}^{\prime}{ } \underline{\tau}-\underline{\sim}^{\prime} C D C \underline{\sim}
\end{aligned}
$$


Assim, pelo Teorema 1, tem-se:

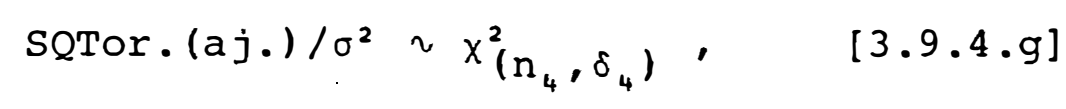

onde:

$$
\begin{aligned}
& n_{4}=v-1 \\
& \delta_{4}=\frac{1}{2 \sigma^{2}}\left(\tau^{\prime} C_{\tau}-\tau^{\prime} C D C \tau\right)
\end{aligned}
$$

3.9 .5 - Distribuição da SQBlocos/ $/ \sigma^{2}$

Sabe-se que:

$$
\begin{aligned}
S Q B & =\underset{\sim}{B} K^{-1} \underline{B}-C_{0} \\
& =\underline{y}^{\prime} X_{3} K^{-1} X_{3}^{\prime} \underline{y}-\underline{\sim} \underline{y}^{\prime} \frac{X_{1} X_{1}^{\prime}}{n} \underset{\sim}{y} \\
& =\underline{y}^{\prime} A_{5} \underline{\underline{y}},
\end{aligned}
$$

onde, $A_{5}=x_{3} K^{-1} x_{3}^{\prime}-\frac{1}{n} x_{1} x_{1}^{\prime}$.

Verifica-se que:

$$
\begin{aligned}
A_{5}^{2}= & x_{3} K^{-1} x_{3}^{\prime} x_{3} K^{-1} x_{3}^{\prime}+\frac{1}{n^{2}} x_{1} x_{1}^{\prime} x_{1} x_{1}^{\prime}-\frac{1}{n} x_{3} K^{-1} x_{3}^{\prime} x_{1} x_{1}^{\prime}- \\
& -\frac{1}{n} x_{1} x_{1}^{\prime} x_{3} K^{-1} x_{3}^{\prime} \\
= & x_{3} K^{-1} K^{-1} x_{3}^{\prime}+\frac{1}{n} x_{1} x_{1}^{\prime}-\frac{1}{n} x_{3} K^{-1} z_{2}^{\prime}-\frac{1}{n} x_{1} z^{\prime} K^{-1} x_{3}^{\prime}
\end{aligned}
$$




$$
\begin{aligned}
& \text { Mas, } x_{3} K^{-1} \underset{z}{z}=x_{1} \text {; então, } \\
& A_{5}^{2}=x_{3} K^{-1} x_{3}^{\prime}+\frac{1}{n} x_{1} x_{1}^{\prime}-\frac{1}{n} x_{1} x_{1}^{\prime}-\frac{1}{n} x_{1} x_{1}^{\prime} \\
& =x_{3} K^{-1} X_{3}^{\prime}-\frac{1}{n} x_{1} x_{1}^{\prime}=A_{5},
\end{aligned}
$$

ou seja, $A_{5}$ ē uma matriz idempotente; e ainda:

$$
\begin{aligned}
\text { Caract. }\left(A_{5}\right) & =\operatorname{TR}\left(x_{3} K^{-1} x_{3}^{\prime}-\frac{1}{n} x_{1} x_{1}^{\prime}\right) \\
& =T R\left(x_{3}^{\prime} x_{3} K^{-1}-\frac{1}{n} x_{1}^{\prime} x_{1}\right) \\
& =T R\left(I_{b}-1\right) \\
& =b-1
\end{aligned}
$$

Tem-se ainda:

$$
\underline{\mu}^{\prime} A_{5} \underset{\sim}{\mu}=\underline{\theta}^{\prime} X^{\prime}\left(x_{3} K^{-1} X_{3}^{\prime}-\frac{1}{n} x_{1} x_{1}^{-1}\right) x \theta
$$

que, por $[3.8 .3 . b]$ e [3.8.1.b], fica:

$$
\begin{aligned}
& \underline{\mu}^{\prime} A_{5} \underline{\sim}=\tau^{\prime} R_{\sim}^{\tau}-\tau^{\prime} C_{\underline{\tau}}+2 \underset{\sim}{\beta}{ }^{\prime}{ }^{\prime} \underset{\sim}{\tau}+\underset{\sim}{\beta}{ }^{\prime} \underline{\underline{\beta}} \\
& \text { Assim, pelo Teorema 1, tem-se: }
\end{aligned}
$$

$$
\mathrm{SQB} / \sigma^{2} \sim x^{2}\left(\mathrm{n}_{5}, \delta_{5}\right),
$$

onde,

$$
\begin{aligned}
& \mathrm{n}_{5}=\mathrm{b}-1 \text {; } \\
& \delta_{5}=\frac{1}{2 \sigma^{2}}\left(\tau^{\prime}{ }^{\prime} \underset{\sim}{\tau}-\underline{\tau}^{\prime} C_{\mathcal{I}}+2 \underline{\beta^{\prime}} N^{\prime} \underline{\tau}+\underline{\beta}^{\prime} K \underline{\sim}\right) .
\end{aligned}
$$


3.9.6 - Distribuição da SQResíduo/ $/ \sigma^{2}$

A SQResíduo é dada por:

$$
\begin{aligned}
S Q R & =\underline{\sim} \underline{y}^{\prime} \underline{\sim}-\tau^{\prime} Q-\underline{B}^{\prime} K^{-1} \underset{\sim}{B}, \\
& =\underline{\sim}^{\prime} \underline{\sim}-\underline{y}^{\prime} X W^{\prime} M^{-1} W X^{\prime} \underset{\sim}{y}-\underline{y}^{\prime} X_{3} K^{-1} X_{3}^{\prime} \underline{\sim} \\
& =\underline{y}^{\prime}\left(I-X W^{\prime} M^{-1} W X^{\prime}-X_{3} K^{-1} X_{3}^{\prime}\right) \underset{\sim}{y}
\end{aligned}
$$

Fazendo $A_{6}=I-X W^{\prime} M^{-1} \mathrm{WX}^{\prime}-\mathrm{X}_{3} \mathrm{~K}^{-1} \mathrm{X}_{3}^{\prime}$, tem-se:

$$
\begin{aligned}
A_{6}^{2}= & I+X W^{\prime} M^{-1} W^{\prime}+X_{3} K^{-1} X_{3}^{\prime}-X W^{\prime} M^{-1} W X^{\prime}-X_{3} K^{-1} X_{3}^{\prime}- \\
& -X W^{\prime} M{ }^{-1} W^{\prime}+X W^{\prime} M^{-1} W X^{\prime} X_{3} K^{-1} X_{3}^{\prime}-X_{3} K^{-1} X_{3}^{\prime}+ \\
& +X_{3} K^{-1} X_{3}^{\prime} X W^{\prime} M^{-1} W X^{\prime} \\
= & I-X W^{\prime} M{ }^{-1} W X^{\prime}-X_{3} K^{-1} X_{3}^{\prime}+X W^{\prime} M{ }^{-1} W X^{\prime} X_{3} K^{-1} X_{3}^{\prime}+ \\
& +X_{3} K^{-1} X_{3}^{\prime} X W^{\prime} M{ }^{-1} W X^{\prime}
\end{aligned}
$$

$$
\text { Mas, } X_{3}^{\prime} X^{\prime}=\left[N^{\prime} K\right] \text {. Então, }
$$$$
X_{3}^{\prime} X W^{\prime}=\left[\begin{array}{ll}
N^{\prime} & K
\end{array}\right]\left[\begin{array}{c}
I_{V^{\prime}} \\
-K^{-1} N^{\prime}
\end{array}\right)=N^{\prime}-N^{\prime}=\underset{\sim}{\phi}
$$ 
Portanto, tem-se que:

$$
A_{6}^{2}=I-X W^{\prime} M^{-1} W X^{\prime}-X_{3} K^{-1} X_{3}^{\prime}=A_{6},
$$

ou seja, $A_{6}$ é uma matriz idempotente; e ainda:

$$
\begin{aligned}
\operatorname{Caract.}\left(A_{6}\right) & =T R\left(I_{n}\right)-T R\left(X W^{\prime} M T^{-1} W X^{\prime}\right)-T R\left(X_{3} K^{-1} X_{3}^{\prime}\right) \\
& =n-T R\left(M^{T^{I}} W X^{\prime} X W^{\prime}\right)-T R\left(X_{3}^{\prime} X_{3} K^{-1}\right) \\
& =n-T R\left(M^{-1} C\right)-T R\left(I_{b}\right) \\
& =n-\left(V^{\prime}-I\right)-b \\
& =n-v^{\prime}-b+1
\end{aligned}
$$

Tem-se tambēm:

$$
\begin{aligned}
& \underline{\sim}^{\prime} A_{6} \underset{\sim}{\mu}=\underline{\theta}^{\prime} X^{\prime}\left(I-X W^{\prime} M{ }^{-1} W X^{\prime}-X_{3} K^{-1} X_{3}^{\prime}\right) X \underset{\sim}{\theta} \\
& =\underline{\sim}^{\prime} X^{\prime} X \underset{\sim}{\theta}-\underline{\sim}^{\prime} X^{\prime} X W^{\prime} M{ }^{-1} W X^{\prime} X \underset{\sim}{\theta}-\underset{\sim}{\theta^{\prime}} X^{\prime} X_{3} K^{-1} X_{3} X \underset{\sim}{\theta} \\
& =\tilde{\theta}^{\prime} X^{\prime} X \tilde{\theta}-\underline{\tau}^{\prime} C^{\prime} M^{-1} C \tau-\underline{\theta}^{\prime} X^{\prime} X_{3} K^{-1} X_{3} X \underline{\theta} \\
& \text { I } \\
& \text { Mas, } C^{\prime} M{ }^{-1} C=C, e \\
& \underline{\sim}^{\prime} X^{\prime} X \underline{\sim} \underset{\sim}{\theta}\left[\begin{array}{ll}
\tau^{\prime} & \underline{\sim}^{\prime}
\end{array}\right]\left(\begin{array}{ll}
R & N \\
N^{\prime} & K
\end{array}\right)\left(\begin{array}{l}
\tau \\
\underset{\sim}{\beta}
\end{array}\right) \\
& =\underline{\tau}^{\prime} R \tau+2 \underset{\sim}{\tau}{ }^{\prime} \underset{\sim}{\beta}+\underline{\sim}^{\prime} K \underset{\sim}{\beta} .
\end{aligned}
$$


Logo, a expressão de [3.9.6.d] fica:

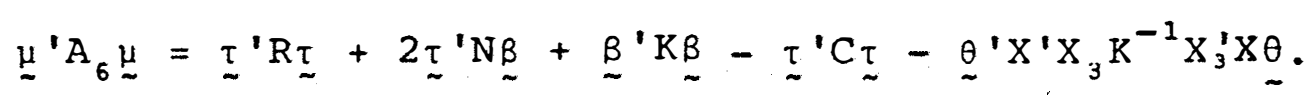

Observa-se por [3.8.3b] que, se o efeito da média não é considerado no modelo, tem-se:

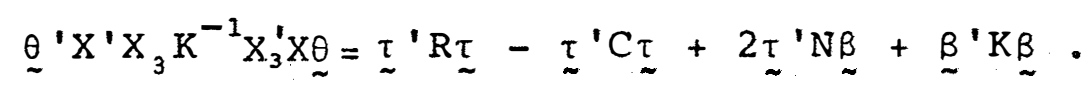

Portanto, segue-se que:

$$
\stackrel{\sim}{\mu} A_{6} \underline{\mu}=0
$$

Assim, pelo Teorema 1, tem-se:

$$
\mathrm{SQR} / \sigma^{2} \sim \mathrm{x}_{\left(\mathrm{n}_{6}, 0\right),}
$$

ou seja, $\mathrm{SQR} / \sigma^{2}$ tem distribuição de qui-quadrado central, com $n_{6}$ graus de liberdade, onde,

$$
\mathrm{n}_{6}=\mathrm{n}-\mathrm{v}^{\prime}-\mathrm{b}+\mathrm{l}
$$

3.9.7 - Distribuição do quociente $\frac{\mathrm{SQTaj} \cdot / \mathrm{n}_{1}}{\mathrm{SQR} / \mathrm{n}_{6}}$

De $[3.9 .1 . a]$ e $[3.9 .6 . a]$, tem-se:

$$
\text { SQTaj. }=\underline{\sim}^{\prime} \mathrm{A}_{1} \underset{\sim}{\mathrm{y}}, \quad \mathrm{SQR}=\underline{\sim}^{\prime} \mathrm{A}_{6} \underset{\sim}{\mathrm{Y}},
$$

respectivamente. 
Verifica-se que, sendo $V=\sigma^{2} I$, então,

$$
\begin{aligned}
& A_{1} V A_{6}= A_{1} A_{6} \sigma^{2} \\
&=\left(X W^{\prime} M^{-1} W X^{\prime}\right)\left(I-X W^{\prime} M^{-1} W X^{\prime}-X_{3} K^{-1} X^{\prime}\right) \sigma^{2} \\
&=\left(X W^{\prime} M^{-1} \cdot W X^{\prime}-X W^{\prime} M^{-1} W X^{\prime}-X W^{\prime} M^{-1} W X^{\prime} X_{3} K^{-1} X^{\prime}\right) \sigma^{2} \\
& \text { Mas, por }[3.9 .6 . b], X_{3}^{\prime} X W^{\prime}=\phi . \text { Logo, } \\
& A_{1} V A_{6}=\phi . \\
& {[3.9 .7 . a] }
\end{aligned}
$$

Assim, pelo Teorema 2, SQTaj. e SQR são inde pendentes; e usando-se o Teorema 3, tem-se, por [3.9.1.e] e $[3.9 .6 . e]$, que:

$$
\frac{\operatorname{sQTaj} \cdot / n_{1}}{\operatorname{sQR} / n_{6}} \sim F_{\left(n_{1}, n_{6}, \delta_{1}\right)}
$$

onde:

$$
\begin{aligned}
& n_{1}=v^{\prime}-1 ; \\
& n_{6}=n-v^{\prime}-b+1 ; \\
& \delta_{1}=\frac{1}{2 \sigma^{2}} I^{\prime} C_{\sim} .
\end{aligned}
$$


3.9 .8 - Distribuição do quociente $\frac{\mathrm{S} Q \mathrm{Tad} . / \mathrm{n}_{2}}{\mathrm{SQR} / \mathrm{n}_{6}}$ De $[3.9 .2 . a]$ e $[3.9 .6 . a]$, tem-se:

SQTad. $=\underline{\sim}^{\prime} \mathrm{A}_{2} \underset{\sim}{\mathrm{y}}$ e $\quad \mathrm{SQR}=\underline{\underline{y}}^{\prime} \mathrm{A}_{6} \underset{\sim}{\mathrm{y}}$,

respectivamente.

E conveniente, no entanto, apresentar-se a SQTad. considerando [3.7.i], ou seja,

$$
\begin{aligned}
& \text { sQTad. }=\frac{1}{b} \underset{s^{\prime}=1}{c} Q_{a_{s^{\prime}}}-\frac{1}{b c}\left(\sum_{s^{\prime}=1}^{c} Q_{a_{s}}\right)^{2} \\
& =\frac{I}{b}{\underset{\sim}{2}}_{2}^{\prime}{\underset{\sim}{2}}_{2}-\frac{I}{\mathrm{bC}}\left(\underset{\sim}{J^{\prime}} \underline{\sim}_{2}\right) \cdot\left({\underset{\sim}{J}}_{\sim_{2}}^{Q_{2}}\right) \\
& =\underset{\sim}{y} X W^{\prime} G W X X^{\prime} \underline{y}
\end{aligned}
$$

onde,

$$
\begin{aligned}
& G=\left(\begin{array}{cc}
\phi & \phi \\
\phi & \frac{I}{b}\left(I-\frac{\underset{\sim}{J J^{\prime}}}{\mathrm{bc}}\right)
\end{array}\right] \\
& \text { Tomando-se } A_{2}=X W^{\prime} G W X^{\prime} \text {, tem-se que: } \\
& A_{2} V A_{6}=\left[X W^{\prime} G W X^{\prime}\left(I-X W^{\prime} M^{-1} W^{\prime}-X_{3} K^{-1} X_{3}^{\prime}\right)\right] \sigma^{2} \\
& =\left[X W^{\prime} G W X^{\prime}-X W^{\prime} G W X^{\prime} X W^{\prime} M{ }^{-1} W X^{\prime}-X W^{\prime} G W X^{\prime} X_{3} K^{-1} X_{3}^{\prime}\right] \sigma^{2}
\end{aligned}
$$




$$
\text { Mas, } \mathrm{wX}^{\prime} \mathrm{XW} \mathrm{W}^{\prime}=\mathrm{C}, \mathrm{e} \text { por }[3.9 .6 . \mathrm{b}], \mathrm{wX}^{\prime} \mathrm{x}_{3}=\phi \text {. }
$$

Logo,

$$
\begin{aligned}
A_{2} V A_{6}= & \left(X W^{\prime} G W X^{\prime}-X W^{\prime} G C M^{-1} W X^{\prime}\right) \sigma^{2} \\
= & {\left[X W^{\prime} G W X^{\prime}-X W^{\prime} G\left(A^{\prime}+M^{\prime}\right) M^{-1} W X^{\prime}\right] \sigma^{2} } \\
= & \left(X W^{\prime} G W X^{\prime}-X W^{\prime} G A^{\prime} M^{-1} W X^{\prime}-X W^{\prime} G W X^{\prime}\right) \sigma^{2} \\
& \text { Como } A^{\prime} M^{T^{1}} W X^{\prime}=\phi, \text { conforme em [3.9.4], se- }
\end{aligned}
$$

gue-se que:

$$
\mathrm{A}_{2} \mathrm{VA}_{6}=\phi
$$

Assim, pelo Teorema 2, a SQTad. e SQR são in dependentes, e usando-se o Teorema 3, tem-se, por [3.9.2.d] e $[3.9 .6 . e]$, que:

$$
\frac{\mathrm{SQTad} . / \mathrm{n}_{2}}{\mathrm{SQR} / \mathrm{n}_{6}} \sim \mathrm{F}\left(\mathrm{n}_{2}, \mathrm{n}_{6}, \delta_{2}\right)
$$

onde,

$$
\begin{aligned}
& \mathrm{n}_{2}=\mathrm{c}-1 ; \\
& \mathrm{n}_{6}=\mathrm{n}-\mathrm{v}^{\prime}-\mathrm{b}+1 ; \\
& \delta_{2}=\frac{1}{2 \sigma^{2}}{\underset{\sim}{2}}_{2}^{\prime}\left(\mathrm{R}_{2}-\frac{\mathrm{b}}{\mathrm{c}} \underset{\sim}{J J^{\prime}}\right) \underset{\sim}{\tau} .
\end{aligned}
$$




$$
3.9 .9 \text { - Distribuição do quociente } \frac{\mathrm{SQTipos} / \mathrm{n}_{3}}{\mathrm{SQR} / \mathrm{n}_{6}}
$$

A SQTipos, por $[3.7 . \ell], e ́$ :

$$
\begin{aligned}
& \text { SQTipos }=\frac{k^{\prime}}{\text { rvc }}\left(\sum_{s=1}^{v} Q_{s}\right)^{2} \\
& =\frac{k^{\prime}}{\operatorname{rvc}}\left(\underline{\sim}^{\prime} \stackrel{\sim}{Q}_{1}^{*}\right)^{\prime}\left({\underset{\sim}{J}}^{\prime}{\stackrel{\star}{Q_{1}}}_{1}\right) \\
& =\underline{\sim}^{\prime} X W^{\prime} F W X^{\prime} \underline{\sim} \quad
\end{aligned}
$$

onde,

$$
F=\left(\begin{array}{cc}
\frac{k^{\prime}}{r v J^{\prime}} & \phi \\
\phi & \phi
\end{array}\right)
$$

Por $[3.9 .6 . a]$, a $S \Omega R$ é:

$$
S Q R=\underline{y}^{\prime} A_{6} \underline{y}
$$

Tomando-rse $\mathrm{A}_{3}=\mathrm{XW}$ 'FWX', verifica-se que:

$$
\begin{aligned}
A_{3} V A_{6}= & \left(X W^{\prime} F W X^{\prime}\right)\left(I-X W^{\prime} M^{-1} W X^{\prime}-X_{3} K^{-1} X_{3}^{\prime}\right) \sigma^{2} \\
= & \left(X W^{\prime} F W X^{\prime}-X W^{\prime} F W X^{\prime} X W^{\prime} M T^{-1} W X^{\prime}-X W^{\prime} F W X^{\prime} X_{3} K^{-1} X^{\prime}\right) \sigma^{2} . \\
& M a{ }_{1}, W X^{\prime} X W^{\prime}=C, \text { e por }[3.9 .6 . b], W X^{\prime} X_{3}=\phi .
\end{aligned}
$$

Logo, 


$$
\begin{aligned}
A_{3} V A_{6}= & \left(X W^{\prime} F W X^{\prime}-X W^{\prime} F C M^{-1} W X^{\prime}\right) \sigma^{2} \\
= & {\left[X W^{\prime} F W X^{\prime}-X W^{\prime} F\left(A^{\prime}+M^{\prime}\right) M^{-1} W X^{\prime}\right] \sigma^{2} } \\
= & {\left[X W^{\prime} F W X^{\prime}-X W^{\prime} F A^{\prime} M^{-1} W X^{\prime}-X W^{\prime} F W X^{\prime}\right] \sigma^{2} }
\end{aligned}
$$

Sendo $A^{\prime} M^{-1} W X^{\prime}=\phi$, segue-se que:

$$
A_{3} V A_{6}=
$$

Assim, pelo Teorema 2, SQTipos e SQR são independentes, e usando-se o Teorema 3, tem-se, por [3.9.3.d] e $[3.9 .6 . e]$, que:

$$
\frac{\text { SQTipos } / \mathrm{n}_{3}}{\mathrm{SQR} / \mathrm{n}_{6}} \sim \mathrm{F}_{\left(\mathrm{n}_{3}, \mathrm{n}_{6}, \delta_{3}\right)}
$$

onde:

$$
\begin{aligned}
& \mathrm{n}_{3}=1 ; \\
& \mathrm{n}_{6}=\mathrm{n}-\mathrm{v}^{\prime}-\mathrm{b}+1 ; \\
& \delta_{3}=\frac{1}{2 \sigma^{2}}\left(\frac{r}{\mathrm{v}} \tau_{-1}^{\prime} \mathrm{J}_{\sim}^{*} \mathcal{\sim}^{*} \tau_{1}+\frac{\mathrm{b}}{\mathrm{c}} \tau_{\sim}^{\prime} \sim_{\sim}^{\prime} \mathcal{\sim}^{\prime} \tau_{2}\right) .
\end{aligned}
$$


3.9 .10 - Distribuição do quociente $\frac{\mathrm{SQTOr}_{\text {. }}(\mathrm{aj} .) / \mathrm{n}_{4}}{\mathrm{SQR} / \mathrm{n}_{6}}$

De $[3.9 .4 . c]$ e [3.9.6.a] tem-se:

$$
\text { SQTor. }\left(\text { aj.) }=\underline{\sim}^{\prime} A_{4} \underline{y} \text { e } \quad S Q R=\underline{y}^{\prime} A_{6} \underline{y}\right. \text {, }
$$

respectivamente.

Verifica-se que, sendo $V=\sigma^{2} I$, então,

$$
\begin{aligned}
A_{4} V_{6}= & \left(X W^{\prime} M^{-1} W X^{\prime}-X W^{\prime} D W X^{\prime}\right)\left(I-X W^{\prime} M^{-1} W X^{\prime}-X_{3} K^{-1} X_{3}\right) \sigma^{2} \\
& \text { Como } W^{\prime} X^{\prime} X W=C \text { e } W X^{\prime} X_{3}=\phi \text {, segue-se que: } \\
A_{4} V A_{6}= & \left(X W^{\prime} M^{-1} W X^{\prime}-X W^{\prime} M^{-1} C M^{-1} W X^{\prime}-X W^{\prime} D W X^{\prime}+X W^{\prime} D C M^{-1} W X^{\prime}\right) c \\
& M a S, C=M^{\prime}+A^{\prime}, X W^{\prime} M^{-1} A^{\prime}=\phi e A^{\prime} M^{-1} W X^{\prime}=\phi !
\end{aligned}
$$

conforme em (3.9.4). Logo,

$$
A_{4} V A_{6}=\left(X W^{\prime} M^{-1} W^{\prime}-X W^{\prime} M^{-1} W X^{\prime}-X W^{\prime} D W X^{\prime}+X W^{\prime} D W X^{\prime}\right) \sigma^{2},
$$

ou seja,

$$
A_{4} \mathrm{VA}_{6}=\phi \quad .
$$

$\checkmark$

Portanto, pelo Teorema 2, SQTor.(aj.) e SQR são independentes, e pelo Teorema 3 , tem-se, por [3.9.4.g] e $[3.9 .6 . e]$, que:

$$
\frac{\text { SQTOr. }(a j .) / n_{4}}{\operatorname{sQR} / n_{6}} \sim F_{\left(n_{4}, n_{6}, \delta_{6}\right)},[3.9 .10 . b]
$$


onde:

$$
\begin{aligned}
& \mathrm{n}_{4}=\mathrm{v}-1 ; \\
& \mathrm{n}_{6}=\mathrm{n}-\mathrm{v}^{\prime}-\mathrm{b}+1 ; \\
& \delta_{6}=\frac{1}{2 \sigma^{2}}\left(\tau^{\prime} \mathrm{C}_{\sim}-\tau^{\prime} \mathrm{CDC} \underset{\sim}{\tau}\right) .
\end{aligned}
$$

3.10 - Estimadores das Médias de Tratamentos Ajustadas pa ra Blocos

As estimativas das médias dos tratamentos, ajustadas para blocos, são obtidas de:

i) Tratamentos originais: $\hat{\mathrm{m}}_{s}=\hat{\mathrm{m}}+\hat{E}_{s},(s=1,2, \ldots, v)$, $[3.10 . a]$

ii) Tratamentos adicionais: $\hat{m}_{a_{s^{\prime}}}=\hat{m}+E_{a_{s^{\prime}}},\left(s^{\prime}=1,2, \ldots, c\right)$. $[3.10 . b]$

3.11 - Quadro da Análise de Variância

$$
\text { O quadro da análise de variância, com as es- }
$$
peranças dos quadrados médios, pode ser apresentado na seguinte forma: 
125.

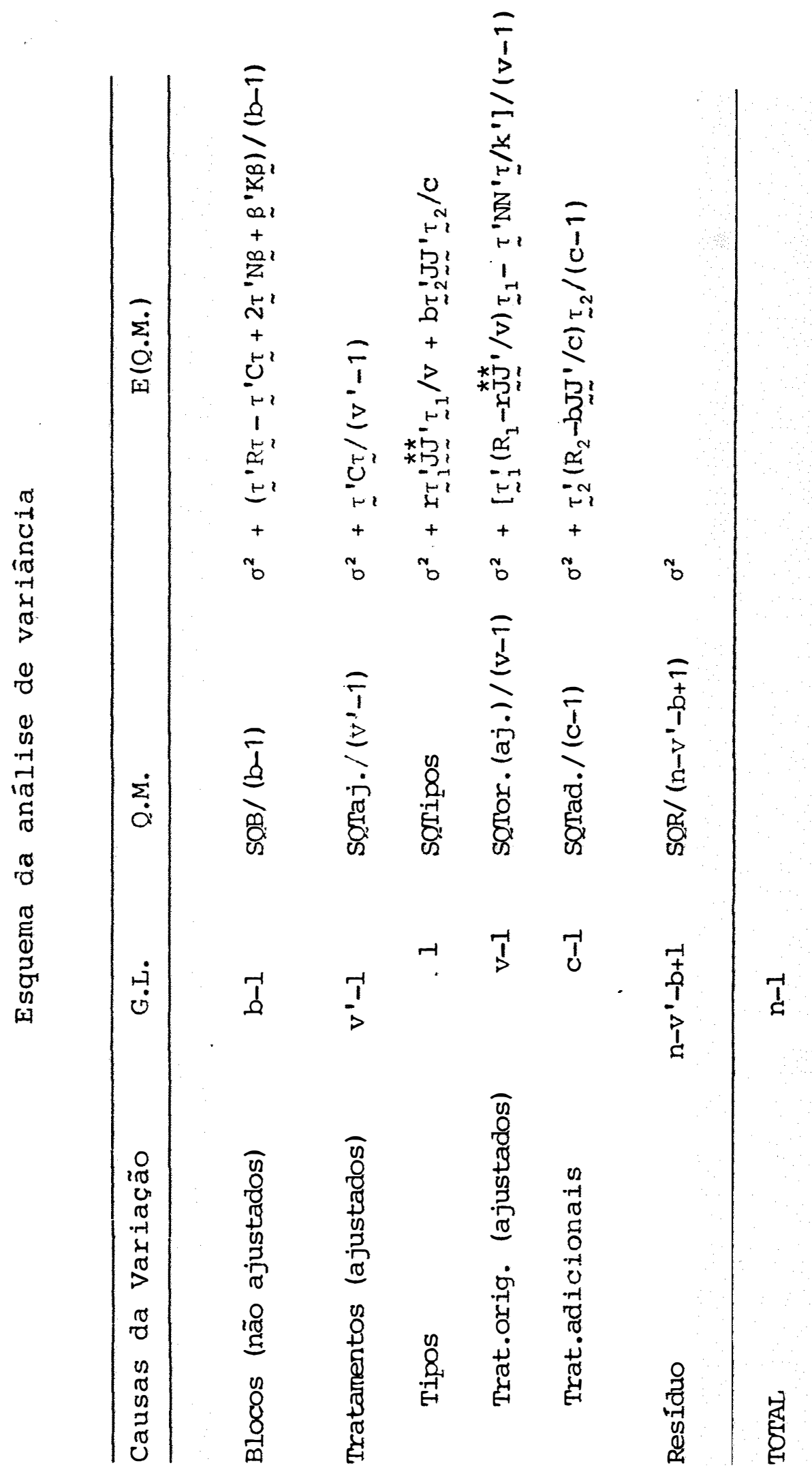


- As somas de quadrados no quadro anterior são:

$S Q B=\frac{1}{k^{\prime}} \sum_{h=1}^{b} B_{h}^{2}-C$

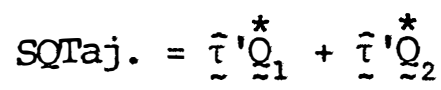

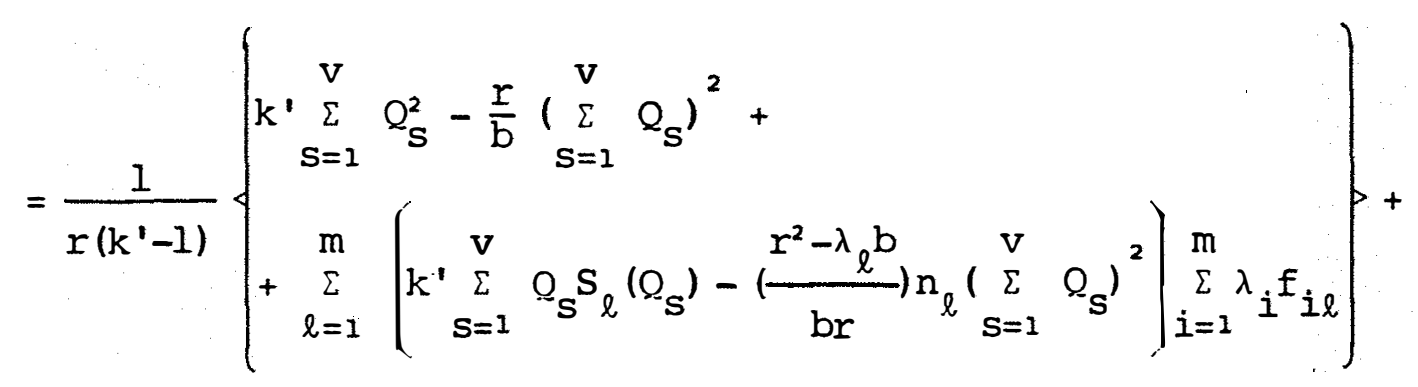

$$
+\frac{1}{b} \sum_{S^{\prime}=1}^{c} Q_{a_{S^{\prime}}}
$$

SQTipos $=\frac{k^{\prime}}{\operatorname{rvc}}\left(\sum_{S=1}^{v} Q_{S}\right)^{2}$

sQTad. $=\frac{1}{b} \underset{s^{\prime}=1}{c} Q_{a_{s^{\prime}}}^{2}-\frac{1}{b c}\left(\sum_{s^{\prime}=1}^{c} Q_{a_{S^{\prime}}}\right)^{2}$

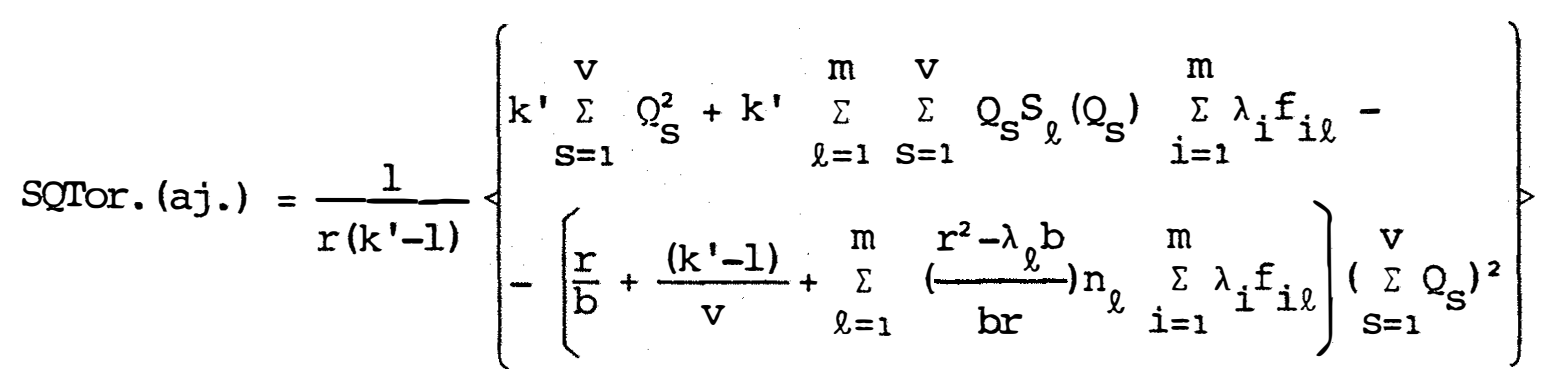

$\mathrm{SQT}=\sum_{\mathrm{u}, \mathrm{h}} \mathrm{Y}_{\mathrm{uh}}^{2}-\mathrm{C}_{0}$

$S Q R=S Q T-S Q B-S Q T a j$. 
A SQTor.(aj.) pode ser obtida mais facilmente através da expressão:

$$
\text { SQTor. }(\text { aj.) = SQTaj. - SQTipos - SQTad. }
$$

3.12 - Variância da Estimativa de um Contraste entre Duas Médias de Tratamentos

Sabe-se, por $[3.6 .2 . \mathrm{k}]$, que a variância de $\underset{\sim}{p}{ }^{\prime} \bar{q}$, sendo $\underset{\sim}{p}$ ' uma função linear estimável, é dada por:

$$
\operatorname{Var}\left(\underline{\sim}^{\prime} \tilde{\tau}\right)=\underline{\sim}^{\prime} M^{-1} \underline{\sim} \sigma^{2},
$$

onde os elementos da matriz M são $m_{s s^{\prime}} m_{a_{s^{\prime}} s^{\prime}} m_{s s^{*}} m_{s s^{\prime}}$ e $m_{S^{\prime} s^{\prime *}}$, definidos em (3.6).

Para o caso em questão, há três tipos de con trastes entre médias de dois tratamentos:

10 TIPO: Contraste envolvendo dois tratamentos originais que são $\ell$-ésimos associados.

Nesse caso tem-se que:

$$
\operatorname{Var}\left(\hat{\mathrm{m}}_{\mathrm{s}}-\overline{\mathrm{m}}_{\mathrm{s}^{*}}\right)=\operatorname{Var}\left(E_{s}-E_{\mathrm{s}^{*}}\right)
$$

Com base em [3.6.2.m], obtém-se: 
128 .

$$
\begin{aligned}
\operatorname{Var}\left(E_{S}-E_{S^{*}}\right) & =\left(m_{S S}+m_{S^{*} S^{*}}-m_{S S^{*}}-m_{S^{*} S}\right) \sigma^{2} \\
& =\left(\frac{k^{\prime}}{r\left(k^{\prime}-1\right)}+\frac{k^{\prime}}{r\left(k^{\prime}-1\right)}-\frac{k^{\prime}}{r\left(k^{\prime}-1\right)} \sum_{i=1}^{m} \lambda_{i} f_{i l}-\frac{k^{\prime}}{r\left(k^{\prime}-1\right)} \sum_{i=1}^{m} \lambda_{i} f_{i l}\right) \sigma^{2} \\
& =\frac{2 k^{\prime}}{r\left(k^{\prime}-1\right)}\left(1-\sum_{i=1}^{m} \lambda_{i} f_{i l}\right) \sigma^{2}
\end{aligned}
$$

Logo,

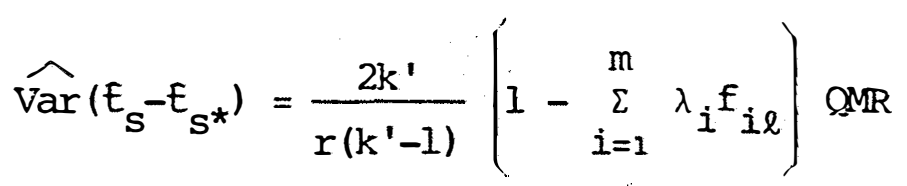

2. TIPO: Contraste envolvendo dois tratamentos adicionais

$$
\operatorname{Var}\left(\bar{m}_{a_{s^{\prime}}}-\bar{m}_{a_{s^{\prime}}}\right)=\operatorname{Var}\left(E_{a_{s^{\prime}}}-E_{a_{s^{\prime}}}\right)
$$

Com base em [3.6.2.m], obtēm-se:

$$
\begin{aligned}
\operatorname{Var}\left(E_{a_{S^{\prime}}}-E_{a_{S^{\prime}} *}\right) & =\left(m_{s^{\prime} s^{\prime}}+m_{a_{S^{\prime}} * s^{\prime} *}-m_{a_{s^{\prime} s^{\prime}} *}-m_{a_{S^{\prime}} * s^{\prime}}\right) \sigma^{2} \\
& =\left(\frac{1}{b}+\frac{1}{b}-0-0\right) \sigma^{2} \\
& =\frac{2 \sigma^{2}}{b} .
\end{aligned}
$$


Logo,

$$
\widehat{\operatorname{Var}}\left(E_{a_{s^{\prime}}}-E_{a_{S^{\prime}}}\right)=\frac{20 M R}{b}
$$

39 TIPO: Contraste envolvendo um tratamento original e outro adicional.

$$
\operatorname{Var}\left(\hat{m}_{s}-\hat{m}_{a_{s^{\prime}}}\right)=\operatorname{Var}\left(E_{s}-\hat{t}_{a_{s^{\prime}}}\right) \text {. }
$$

Com base em $[3.6 .2 . \mathrm{m}]$, tem-se:

$$
\begin{aligned}
\operatorname{Var}\left(E_{s}-E_{a_{s^{\prime}}}\right) & =\left(m_{s s}+m_{a_{s^{\prime} s^{\prime}}}-m_{s^{\prime}}-m_{s^{\prime} s^{\prime}}\right) \sigma^{2} \\
& =\left\langle\frac{k^{\prime}}{r\left(k^{\prime}-1\right)}+\frac{1}{b}-\frac{1}{r\left(k^{\prime}-1\right)}\left(\frac{r}{b}+\sum_{l=1}^{m}\left(\frac{r^{2}-\lambda_{\ell} b}{b r}\right) n_{\ell} \sum_{i=1}^{m} \lambda_{i} f_{i \ell}\right)-0\right\} \sigma^{2}
\end{aligned}
$$

Logo,

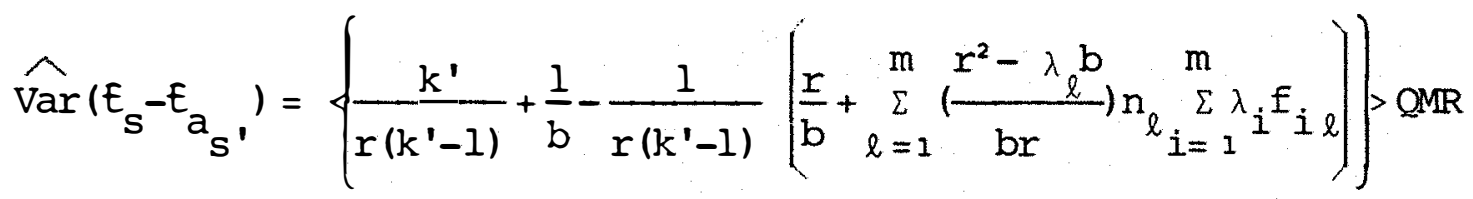

Considerando-se 0 caso especial onde $\lambda_{1}=\lambda_{2}=$ $=\ldots=\lambda$, ou seja, tratando-se de delineamentos BIB, as variâncias anteriores modificam-se para: 


$$
\begin{aligned}
& \operatorname{Var}\left(\hat{m}_{s^{-}}-\bar{m}_{s^{*}}\right)=\frac{2 k^{\prime} \sigma^{2}}{\lambda v+r c}, \\
& \operatorname{Var}\left(\hat{m}_{a_{s^{\prime}}}-\bar{m}_{a_{s^{\prime}}}\right)=\frac{2 \sigma^{2}}{b}, e \\
& \operatorname{Var}\left(\hat{m}_{s^{-}}-\hat{m}_{a_{s^{\prime}}}\right)=\left(\frac{k^{\prime}}{\lambda v+r c}+\frac{1}{b}-\frac{k^{\prime}}{n(\lambda v+r c)} \frac{\left(r^{2}-\lambda b\right)}{r}\right) \sigma^{2}
\end{aligned}
$$

as quais concordam com os resultados obtidos por KÄLIN (1966) e FERREIRA (1980).

3.13-Testes de Significâncias

3.13.1 - Teste de significância para tratamentos ajuㅗ tados para blocos

Pode-se verificar, por $[3.9 .7 . b]$, que:

$$
\frac{\operatorname{sQTaj} \cdot / n_{1}}{\operatorname{SQR} / n_{6}} \sim F_{\left(n_{1}, n_{6}, \delta_{1}\right)^{\prime}}
$$

onde: $\mathrm{n}_{1}=\mathrm{v}^{\prime}-1$;

$$
\begin{aligned}
& \mathrm{n}_{6}=\mathrm{n}-\mathrm{v}^{\prime}-\mathrm{b}+1 ; \\
& \delta_{1}=\frac{1}{2 \sigma^{2}} \beth^{\prime} \mathrm{C} \tau
\end{aligned}
$$

Portanto, a hipótese de nulidade de que todos os efeitos de tratamentos sejam nulos, isto é,

$$
\mathrm{H}_{0}^{(1)}: \underline{\tau}=\Phi \text {, }
$$


pode ser testada utilizando-se o teste F'central através da estatistica:

$$
F_{1}=\frac{\operatorname{seTaj} \cdot / n_{1}}{\operatorname{sQR} / n_{6}} \text {, }
$$

com $n_{1}$ e $n_{6}$ graus de liberdade, visto que sob a hipótese $H_{0}^{(1)}$ tem-se $\delta_{1}=0$.

3.13.2 - Teste de significância para tratamentos adicio nais

De $[3.9 .8 . c]$ tem-se:

SQTad. $/ \mathrm{n}_{2}$

$\overline{S Q R / n_{6}} \sim_{F}\left(n_{2}, n_{6}, \delta_{2}\right)^{\prime}$

onde: $\mathrm{n}_{2}=\mathrm{c}-1$;

$\mathrm{n}_{6}=\mathrm{n}-\mathrm{v}^{\prime}-\mathrm{b}+1 ;$

$\delta_{2}=\frac{1}{2 \sigma^{2}} \tau_{2}^{\prime}\left(R_{2}-\frac{b}{C} J J_{\sim}^{\prime}\right){\underset{\sim}{2}}_{2}$.

,

Assim, a hipótese de nulidade de que todos efeitos dos tratamentos adicionais sejam nulos, isto é:

$$
\mathrm{H}_{0}^{(2)}: I_{I_{2}}=\Phi,
$$


pode ser testada utilizando-se o teste $F$ central, através da estatística:

$$
\mathrm{F}_{2}=\frac{\mathrm{SQTad} \cdot / \mathrm{n}_{2}}{\mathrm{SQR} / \mathrm{n}_{6}},
$$

com $\mathrm{n}_{2}$ e $\mathrm{n}_{6}$ graus de liberdade, visto que sob a hipótese $\mathrm{H}_{0}^{(2)}, \delta_{2}=0$.

3.13.3 - Teste de significância para tipos de tratamen tos

Observa-se por $[3.9 .9 . c]$ que

SQTipos/n 3

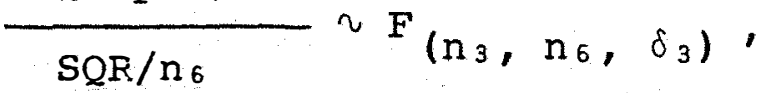

onde: $\mathrm{n}_{3}=1$;

$$
\begin{aligned}
& n_{6}=n-v^{\prime}-b+1 ;
\end{aligned}
$$

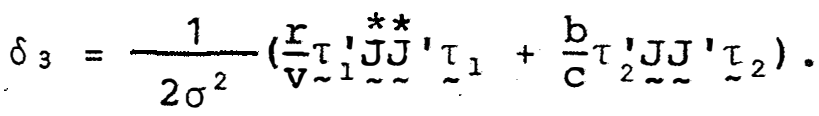

A hipótese de nulidade de que a diferença entre o efeito méaio dos tratamentos originais e o efeito médio dos tratamentos adicionais seja nula, isto é,

$$
\mathrm{H}_{0}^{\left({ }^{3}\right)}: \frac{1}{\mathrm{~V}} \stackrel{\sim}{\prime}^{*} \tau_{\mathcal{I}}=\frac{1}{\mathrm{C}} \mathrm{J}_{\sim}^{\prime} \tau_{2^{\prime}}
$$


pode ser testada utilizanòo-se o teste $F$ central, através da estatistica:

$$
F_{3}=\frac{\text { SQTipos } / \mathrm{n}_{3}}{\mathrm{SQR} / \mathrm{n}_{6}},
$$

com $n_{3}$ e $n_{6}$ graus de liberdade. Pode-se verificar que sob a hipótese $\mathrm{H}_{0}^{(\dot{3})}$ e sob a restrição paramétrica imposta em 3.2 , tem-se que $\delta_{3}=0$.

3.13.4- Teste de significância para tratamentos origi nais ajustados para blocos

De $[3.9 .10 . b]$ tem-se:

$$
\frac{\text { SQTor. }(a j .) / n_{4}}{\operatorname{SQR} / n_{6}} \sim F_{\left(n_{3}, n_{6}, \delta_{6}\right)^{\prime}}
$$

onde: $\mathrm{n}_{4}=\mathrm{v}-1$;

$$
\begin{aligned}
& \mathrm{n}_{6}=\mathrm{n}-\mathrm{v}^{\prime}-\mathrm{b}+1 ; \text {, }
\end{aligned}
$$

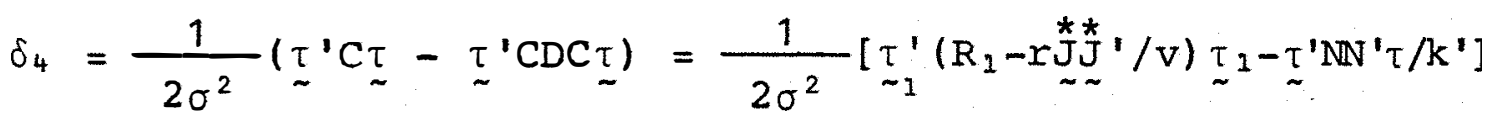

Assim, a hipótese de nulidade de que todós os efeitos dos tratamentos originias sejam nulos, isto é,

$$
\mathrm{H}_{0}^{(4)}: \underset{\tau_{I}}{\tau^{(4}}=
$$


pode ser testada através do tesde F central, tomando-se a es tatistica:

$$
F_{4}=\frac{\text { SQTor. }(a j .) / n_{4}}{S Q R / n_{6}}
$$

com $n_{4}$ e $n_{6}$ graus de liberdade, visto que sob a hipótese $\mathrm{H}_{0}^{\left({ }^{4}\right)}$ e sob a restrição paramétrica, imposta no modelo matemático , tem-se $\delta_{6}=0$. 
4. ILUSTRAÇÃO DO MÉTODO PROPOSTO

Como exemplo de aplicação do método proposto, será utilizado um experimento em reticulado quadrado triplo de 16 tratamentos, mais 2 tratamentos adicionais (testemunhas) em cada bloco, cujos dados encontram-se na Tabela 1. Os dados dos tratamentos originais foram obtidos de PIMENTEL GOMES (1973), e referem-se às produções de híbridos de milho, e os referentes aos tratamentos adicionais são ficti cios. 
Tabela 1 - Produções, em kg/parcela, de híbridos de milho e testemunhas fictícias. (Os nümeros entre parêntesis indicam os tratamentos originais e as letras os tratamentos adicionais).
(1) 2,0
(2) 2,9
(3) 2,2
(4) 3,9
(A) 3,0
$\left(A_{2}\right) 3,2$
17,2
(5) 2,3
(6) 2,5
(7) 1,4
(8) 1,7
(A) 2,8
$\left(A_{2}\right) \quad 2,6$
13,3
(9) 1,6
(10) 3,0
(11) 1,5
(12) 2,1
$\left(A_{1}\right) \quad 2,0$
$\left(A_{2}\right) \quad 2,2$
12,4
(13) 2,3
(14) 3,4
(I5) 2,0
(16) 2,8
$\left(A_{1}\right) \quad 2,5$
$\left(A_{2}\right) \quad 3,2$
16,2
59,1
(1) 2,2
(5) 2,3
(9) 2,7
(13) 1,4
( $\left.A_{1}\right) \quad 2,8$
$\left(A_{2}\right) \quad 2,9$
14,3
(2) 3,1
(6) 2,8
(10) 2,6
(14) 2,8
$\left(A_{1}\right) 3,2$
$\left(A_{2}\right) \quad 3,4$
17,9
(3) 3,1
(7) 2,9
(II) 2,5
(15) 2,4
$\left(A_{1}\right) 3,0$
$\left(A_{2}\right) \quad 3,2$
17,1
(4) 4,0
(8) 2,8
(12) 2,7
(16) 1,5
$\left(A_{1}\right) \quad 4,0$
$\left(A_{2}\right) \quad 3,8$
18,8
68,1

\begin{tabular}{rrrrrrrrrrrrr} 
(1) 3,0 & $(6)$ & 2,9 & (11) 2,6 & $(16)$ & 3,1 & $\left(A_{1}\right)$ & 3,0 & $\left(A_{2}\right)$ & 3,2 & 17,8 \\
(2) 1,8 & $(5)$ & 1,9 & $(12)$ & 2,9 & $(15)$ & 2,5 & $\left(A_{1}\right)$ & 3,2 & $\left(A_{2}\right)$ & 2,8 & 15,1 \\
(3) 1,7 & $(8)$ & 2,0 & $(9)$ & 1,4 & $(14)$ & 2,3 & $\left(A_{1}\right)$ & 2,5 & $\left(A_{2}\right)$ & 2,0 & 11,9 \\
(4) & 4,4 & $(7)$ & 3,7 & $(10)$ & 3,3 & $(13)$ & 2,2 & $\left(A_{1}\right)$ & 3,8 & $\left(A_{2}\right)$ & 3,5 & 20,9 \\
\hline
\end{tabular}


O reticulado quadrado, delineamento utilizado no exemplo, é do tipo quadrado latino com $i=3$ restrições. Logo, de acordo com o que foi relatado em $[2.2 .2 .3]$, há $v=s^{2}=$ =16 tratamentos que satisfazem o seguinte esquema de associação:

$\begin{array}{rrrr}1 a & 2 b & 3 c & 4 d \\ 5 b & 6 a & 7 d & 8 c \\ 9 c & 10 d & 11 a & 12 b \\ 13 d & 14 c & 15 b & 16 a\end{array}$

A regra de associação é a seguinte: Dois trą tamentos são primeiros associados se e somente se eles ocor rem na mesma linha ou coluna do conjunto, ou nas posições ocupadas pela mesma letra. Em outros casos eles são segundos associados.

Sabe-se ainda, por [2.2.2.3], que em um deli neamento PBIB do tipo quadrado latino, as seguintes relações se verificam:

$$
\begin{array}{ll}
\mathrm{n}_{1}=i(s-1), & \mathrm{n}_{2}=(s-1)(s-i+1), \\
p_{11}^{1}=i^{2}-3 i+s, & p_{12}^{1}=p_{21}^{1}=(i-1)(s-i+1), \quad p_{22}^{1}=(s-i)(s-i+1) \\
p_{11}^{2}=i(i-1), & p_{12}^{2}=p_{21}^{2}=i(s-i), \quad p_{22}^{2}=(s-i)^{2}+i-2 .
\end{array}
$$

Pode-se então verificar que os parâmetros do primeiro tipo, referentes ao delineamento PBIB utilizado, são: 


$$
\begin{gathered}
v=16, b=12, \quad r=1=3, \quad k=s=4, \lambda_{1}=1, \lambda_{2}=0, \\
n_{1}=9, n_{2}=6,
\end{gathered}
$$

e os parâmetros do segundo tipo são:

$$
\begin{aligned}
& p_{11}^{1}=4, \quad p_{12}^{1}=p_{21}^{1}=4, \quad p_{22}^{I}=2 \\
& p_{11}^{2}=6, \quad p_{12}^{2}=p_{21}^{2}=3, p_{22}^{2}=2
\end{aligned}
$$

Com a inclusão dos $\mathrm{c}=2$ tratamentos adicionais resulta que:

$$
v^{\prime}=v+c=18 \quad \text { e } \quad k^{\prime}=k+c=6
$$

A análise de variância pode ser desenvolvida com o auxílio da seguinte tabela: 


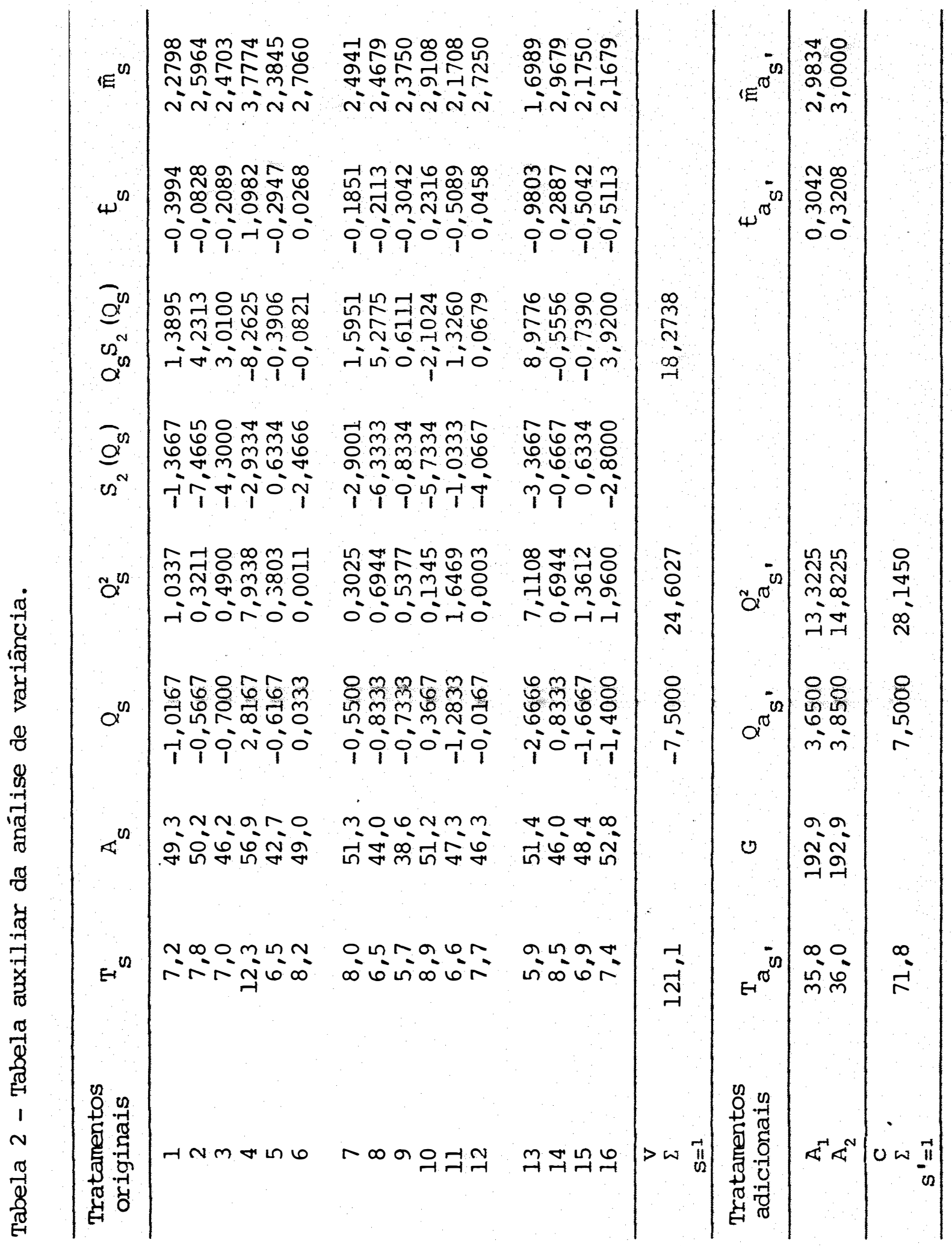


Os efeitos dos tratamentos adicionais foram estimados utilizando-se a expressão [3.5.2.1.e], ou seja,

$$
E_{a_{s^{\prime}}}=\frac{1}{b} Q_{a_{s^{\prime}}},
$$

e os efeitos dos tratamentos originais através de [3.5.2.3.k], ou seja:

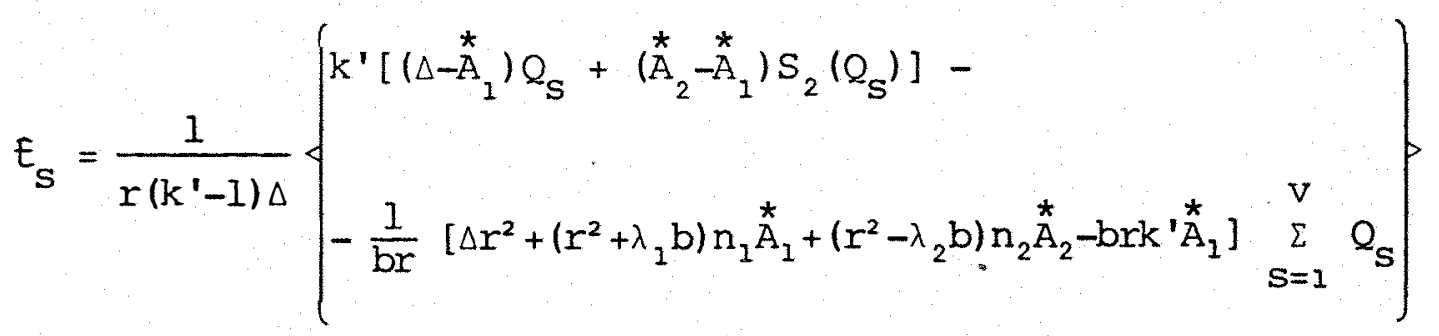

Na expressão anterior sabe-se, de [3.5.2.3],

que:

$$
\text { i) } \Delta=a_{11} a_{22}-a_{12} a_{21} \text {, }
$$

onde,

$$
\begin{aligned}
& a_{11}=r\left(k^{\prime}-1\right)+\left(\lambda_{1}-\lambda_{2}\right) p_{12}^{1}+\lambda_{1} \\
& a_{12}=\left(\lambda_{1}-\lambda_{2}\right) p_{12}^{2} \\
& a_{21}=\left(\lambda_{2}-\lambda_{1}\right) p_{21}^{1} \\
& a_{22}=r\left(k^{\prime}-1\right)+\left(\lambda_{2}-\lambda_{1}\right) p_{21}^{2}+\lambda_{2}
\end{aligned}
$$

Logo, no exemplo, tem-se:

$$
\begin{aligned}
& a_{11}=20, a_{12}=3, \quad a_{21}=-4, \quad a_{22}=12 \\
& \Delta=252
\end{aligned}
$$


ii) $\stackrel{\star}{A}_{1}=\lambda_{1} a_{22}-\lambda_{2} a_{21}$ e $\stackrel{\star}{A}_{2}=\lambda_{2} a_{11}-\lambda_{1} a_{12}$

Logo, no exemplo, tem-se:

$$
\stackrel{\star}{A}_{1}=12 \text { e } \AA_{2}^{\star}=-3 \text {. }
$$

Pode-se verificar então que a expressão para os efeitos dos tratamentos originais estimados fica:

$$
E_{s}=\frac{1}{3.780}\left[1.440 Q_{s}-90 s_{2}\left(Q_{s}\right)-168,750\right] .
$$

As somas de quadrados de tratamentos são:

i) Soma de quadrados dos tratamentos adicionais

De [3.7.i] tem-se:

$$
\begin{aligned}
& \text { SQTad. }=\frac{1}{b} \underset{s^{\prime}=1}{c} Q_{a_{s^{\prime}}}^{c}-\frac{1}{b c}\left(\sum_{s^{\prime}=1}^{c} Q_{a_{s^{\prime}}}\right)^{2} \\
& \text { Logo, } \\
& \text { sQTad. }=\frac{1}{12}(28,145)-\frac{1}{24}(7,5)^{2},
\end{aligned}
$$

ou seja,

$$
\text { SQTad. }=0,001667 \text {. }
$$


ii) Soma de quadrados entre tipos de tratamentos De $[3.7 . l]$ tem-se:

$$
\text { SQTipos }=\frac{k^{\prime}}{r v c}\left(\begin{array}{ll}
\sum_{S=1} & \left.Q_{S}\right)^{2}
\end{array}\right.
$$

Logo,

$$
\text { SQTipos }=\frac{6}{96}(-7,5)^{2} \text {, }
$$

ou seja,

$$
\text { SQTipos }=3,515625 \text {. }
$$

iii) Soma de quadrados dos tratamentos originais ajustada

$$
\begin{aligned}
& \text { De [3.7.q] tem-se: }
\end{aligned}
$$

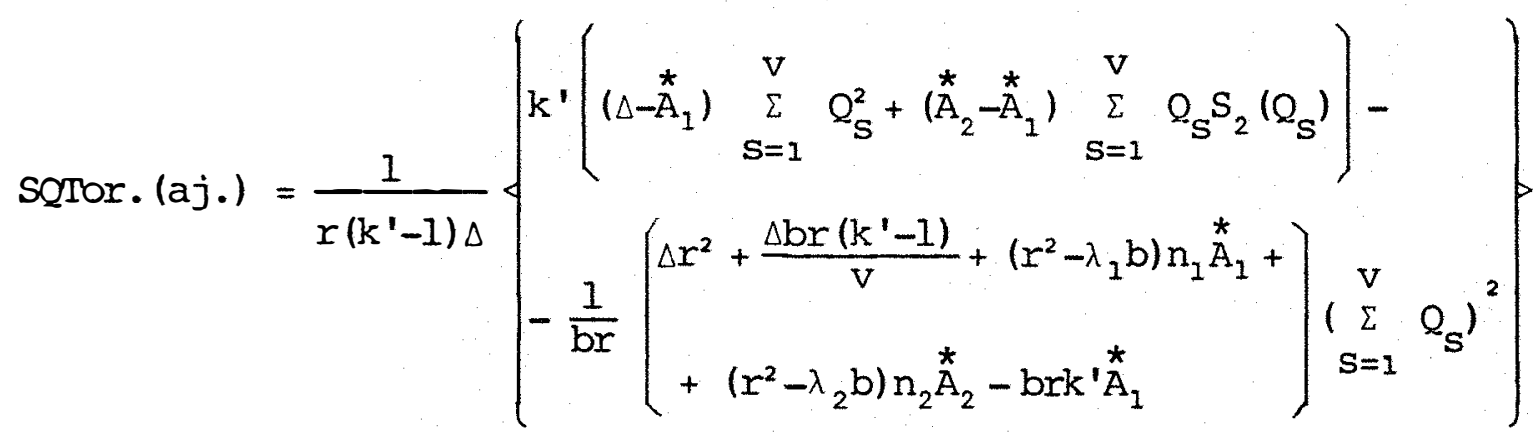

Considerando-se então os dados do exemplo re sulta que:

$$
\text { SQTor. }(a j .)=8,100313 \text {. }
$$


Pode-se verificar que:

$$
\text { SQTaj. = } \tilde{I}^{\prime} Q=\text { SQTad. + SQTipos + SQTor. , }
$$

ou seja,

$$
\text { SQTaj. = 11,617605. }
$$

As demais somas de quadrados são:

$$
\begin{aligned}
& \mathrm{SQT}=\sum_{\mathrm{u}, \mathrm{h}}^{\sum} \mathrm{Y}_{\mathrm{uh}}^{2}-\mathrm{C}_{0}, \\
& \mathrm{SQB}=\frac{1}{\mathrm{~K}} \underset{\mathrm{h}=1}{\mathrm{~b}} \mathrm{~B}_{\mathrm{h}}^{2}-\mathrm{C}_{0}, \mathrm{e} \\
& \mathrm{SQR}=\mathrm{SQT}-\mathrm{SQTaj} \cdot \mathrm{SQB},
\end{aligned}
$$

que resultaram em:

$$
\begin{aligned}
& \mathrm{SQT}=33,158750 \\
& \mathrm{SQB}=13,680417 \\
& \mathrm{SQR}=7,860728
\end{aligned}
$$

A soma de quadrados de blocos pode ainda, pa ra o caso do exemplo, ser decomposta em duas partes: soma de quadrados de repetições (SQRep.) e soma de quadrados de blo cos dentro de repetições (SQB/Rep.). Estas somas de quadra- 
dos, obtidas pela maneira usual, resultaram em:

$$
\begin{aligned}
\text { SQRep. } & =1,810000 \\
\text { SQB /Rep. } & =11,870417
\end{aligned}
$$

Assim, o quadro da análise de variāncia pođe

\begin{tabular}{|c|c|c|c|c|}
\hline Causas da Variação & G.L. & $S . Q$ & Q.M. & $\mathrm{F}$ \\
\hline Blocos (não aj.) & 11 & 13,680417 & 1,243584 & \\
\hline Repetições & 2 & 1,810000 & 0,905000 & \\
\hline Blocos/Rep. & 9 & 11,870417 & 1,318935 & \\
\hline Tratamentos (aj.) & 17 & 11,617605 & 0,683389 & $3,74 * *$ \\
\hline Tipos & 1 & 3,515625 & 3,515625 & $19,23 * *$ \\
\hline Trat.orig. (aj.) & 15 & 8,100313 & 0,540021 & $2,95 * *$ \\
\hline Trat.adic. & 1 & 0,001667 & 0,001667 & $<1,00$ \\
\hline Residuo & 45 & 7,860728 & 0,182808 & \\
\hline Total & 71 & 33,158750 & & \\
\hline
\end{tabular}
ser apresentado como a seguir:

As estimativas das variâncias das estimativas dos contrastes entre efeitos de tratamentos, calculadas com base em [3.12], são: 
i) Contraste envolvendo dois tratamentos originais que são primeiros associados

De [3.12.a] segue-se que:

$$
\widehat{\operatorname{var}}\left(E_{S^{-}}-E_{S^{*}}\right)=\frac{2 k^{\prime} Q M R}{r\left(k^{\prime}-1\right)}\left[1-\left(\lambda_{1} E_{11^{+}} \lambda_{2} E_{21}\right)\right],
$$

onde, de $[3 \cdot 5 \cdot 2 \cdot 3 . f]$,

$$
f_{11}=\frac{a_{22}}{\Delta}=0,047619, \text { e } f_{21}=-\frac{a_{21}}{\Delta}=0,015873
$$

Tem-se então que:

$$
\widehat{\operatorname{var}}\left(E_{S}-E_{S^{*}}\right)=0,139282 \text {. }
$$

ii) Contraste envolvendo dois tratamentos originais que são segundos associados

$$
\text { De }[3.12 . a] \text {, tem-se: }
$$

$$
\widehat{\operatorname{Var}}\left(E_{S}-E_{S^{*}}\right)=\frac{2 k^{\prime} Q M R}{r\left(k^{\prime}-1\right)}\left[1-\left(\lambda_{1} f_{12}+\lambda_{2} f_{22}\right)\right],
$$

onde, de $[3.5 \cdot 2 \cdot 3 . f]$,

$$
f_{12}=-\frac{a_{12}}{\Delta}=-0,011905, e f_{22}=\frac{a_{11}}{\Delta}=0,079365 .
$$


Segue-se então que:

$$
\widehat{\operatorname{Var}}\left(E_{S}-E_{S^{*}}\right)=0,147987
$$

iii) Contraste envolvendo dois tratamentos adicionais

De $[3.12 . b]$ sabe-se que:

$$
\widehat{\operatorname{Var}}\left(E_{a_{s}}-E_{a_{s^{\prime}} *}\right)=\frac{2 Q M R}{b}
$$

Logo,

$$
\widehat{\operatorname{var}}\left(E_{a_{s^{\prime}}}-E_{a_{s^{\prime}} *}\right)=0,030468 .
$$

iv) Contraste envolvendo um'tratamento original e outro adicional

$$
\text { Por }[3.12 . c] \text { tem-se: }
$$

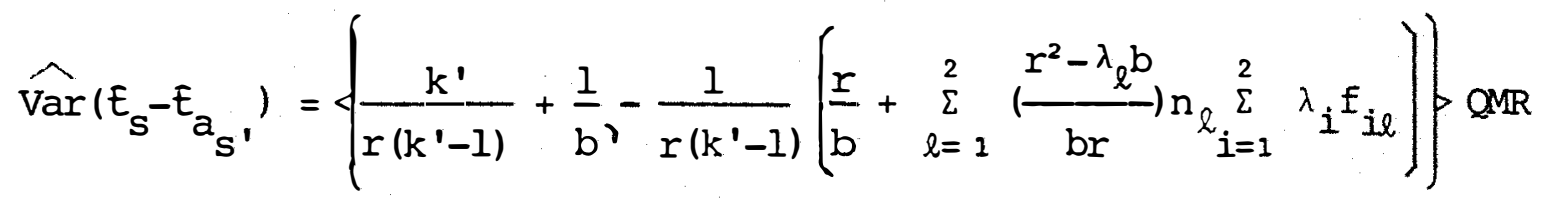

Logo, segue-se que:

$$
\widehat{\operatorname{Var}}\left(E_{s}-E_{a_{s^{\prime}}}\right)=0,085963 .
$$


5. CONCLUSÖES

Em geral, os resultados obtidos permitem as seguintes conclusões:

a) Quanto ao modelo matemático

0 modelo matemático apresentou-se adequado aos objetivos propostos, permitindo a avaliação dos efeitos dos tratamentos originais e dos tratamentos adicionais.

b) Quanto à estimação dos efeitos dos tratamentos

b.1) Os efeitos dos tratamentos adicionais, em delineamen to PBIB, são estimados através do mesmo estimador dos efeitos desses tratamentos, quando em delineamento BIB. 
b.2) A estimação dos efeitos dos tratamentos através do método simplificado facilita a computação dos dados, uma vez que, ao contrário ảo método clássico, não exige a inversão de matrizes de grandes dimensões.

b.3) Os efeitos dos tratamentos originais são mais facilmente estimados, à medida em que diminui o número de classes de associados.

b.4) Os estimadores dos efeitos dos tratamentos originais em delineamento BIB, podem ser obtidos, a partir dos estimadores dos efeitos desses tratamentos em delineamento $\mathrm{PBIB}$, tomando-se $\lambda_{1}=\lambda_{2}=\ldots \lambda_{\mathrm{m}}=\lambda$.

c) Quanto às somas de quadrados

c.1) A soma de quadrados de tratamentos, ajustada para os efeitos de blocos, pode ser decomposta em: Soma de quadrados de tratamentos originais ajustada para blo cos, soma de quadrados de tratamentos adicionais e soma de quadrados entre tipos de tratamentos.

c.2) As demais somas de quadrados são obtidas de modo usual. 
d) Quanto à variância da estimativa de um contraste entre duas médias de tratamentos

d.1) A precisão da estimativa de um contraste entre duas médias de tratamentos originais, depende da classe de associados que esses tratamentos pertencem.

d.2) Quaisquer contrastes entre duas méåias de tratamen tos adicionais são estimados com a mesma precisão.

d.3) Quaisquer constrastes entre duas méäias, envolvendo um tratamento original e outro adicional, são estima dos com a mesma precisão.

e) Quanto aos testes de significância

Os testes $\mathrm{F}$ para os tratamentos originais, tratamentos adicionais e tipos de tratamentos são exatos. 
6. REFERENCIAS BIBLIOGRÁFICAS

BOSE, R.C. e K.R. NAIR, 1939. Partially balanced incomplete block designs. Sankhyã, Calcutã, 4 : 337-372.

BOSE, R.C. e T. SHIMAMOTO, 1952. Classification and analysis of partially balanced incomplete block designs with two associate classes. Journal of the American Statistical Association, Washington, 47: 151-184.

BOSE, R.C. e W.H. CLATWORTHY, 1955. Some classes of partially balanced designs. Annals of Mathematical Statistics, Baltimore, 26: 212-232.

BOSE, R.C. e W.S. CONNOR, 1952. Combinatorial properties of group divisible incomplete block designs. Annals of Mathematical Statistics, Baltimore, 23: 367-383. 
CHAKRABARTI, M.C., 1962. Mathematics of Design and Analysis of Experiments. Asia Publishing House, Iondres. $120 \mathrm{p}$.

COCHRAN, W.G. e G.M. COX, 1957. Experimental Designs. 2a ed., John Wiley \& Sons, Nova York. $611 \mathrm{p}$.

FEDERER, W.T., 1956. Augmented (or Hoonuiaku) designs. The Hawaiian Planters' Record, 55: 191-208.

FEDERER, W.T., 1961. Augmented designs with one-way elimination of heterogeneity. Biometrics, Raleigh, 17: 447-473 .

FERREIRA, J.G., 1980. Análise intrablocos de um experimento em blocos incompletos equilibrados, aumentado pela adição de alguns tratamentos comuns a todos os blocos. Pí racicaba, ESALQ/USP, 57 p. [Dissertação de Mestrado].

FISHER, R.A., 1925. Statistical Methods for Research Workers. Oliver and Boyd, Edinburgh.

GRAYBILL, F.A., 1961. An Introduction to Linear Statistical. Models. McGraw-Hill, Nova York. 463 p.

HARSHBARGER, B., 1949. Triple rectangular lattices. Biometrics, Raleigh, 5: 1-13.

IEMMA, A.F., 1981. Análise de experimentos em parcelas sub divididas com tratamentos principais dispostos em blocos incompletos balanceados. Piracicaba, ESALQ/USP, $145 \mathrm{p}$. [Tese de Doutorado]. 
IEMMA, A.F., 1985. Modelos Lineares - Uma Introdução para Profissionais da Pesquisa Agropecuária. ESALQ/USP, Piracicaba, 142 p. [10 Simpósio de Estatística Aplicada à Ex perimentação Agronômica] .

KÄLIN, A., 1966. Versuchsanordnungen in unvollständigen Blöcken mit zusätzlichen kontrollbehandlungen in jeden Block. Metrika, 10: 182-218.

NAIR, K.R., 1944. The recovery of inter-block information in incomplete block designs. Sankhyã, Calcutá, 6: 383-390 .

NAIR, K.R., 1951. Rectangular lattices and partially balanced incomplete block designs. Biometrics, Raleigh, 7: $145-154$.

NAIR, K.R., 1952. Analysis of partially balanced incomplete block designs illustrated on the simple square and rectangular lattices. Biometrics, Raleigh, 8: 122-155.

OGAWA, J., 1974. Statistical Theory of Analysis of Experimental Designs. Marcel Dekker in., Nova York. 465 p.

PAVATE, M.V., 1961. Combined analysis of balanced incomple te block designs with some common treatments. Biometrics." Raleigh, 17: 111-119.

PIMENTEL GOMES, F., 1967. The solution of normal equations of experiments in incomplete blocks. Ciência e Cultura, São Paulo, 20: 733-746. 
PIMENTEL GOMES, F.., i973, Curso de Estatistica Experimental. 5a ed., Livraria Nobel, Piracicaba. $430 \mathrm{p}$.

RAGHAVARAO, D., 1971. Combinatorial Problems in Design of Experiments. Willey, Nova York. 386 p.

RAO, C.R., 1947. General methods of analysis for incomplete block designs. Journal of the American Statistical Association, Washington, 58: 541-561.

SEARLE, S.R., 1971. Linear Models.Willey, Nova York. 531 p.

SHRIKHANDE, S.S., 1952. On the dual of some balanced incom plete block designs. Biometrics, Raleigh, 8: 66-72.

YATES, F., 1936. Incomplete randomized blocks. Annals of Eugenics, Londres, 7: 121-140. 